\title{
WestVirginiaUniversity
}

THE RESEARCH REPOSITORY @ WVU

Graduate Theses, Dissertations, and Problem Reports

2013

\section{Connectivity and spanning trees of graphs}

\author{
Xiaofeng Gu \\ West Virginia University
}

Follow this and additional works at: https://researchrepository.wvu.edu/etd

\section{Recommended Citation}

Gu, Xiaofeng, "Connectivity and spanning trees of graphs" (2013). Graduate Theses, Dissertations, and Problem Reports. 4970.

https://researchrepository.wvu.edu/etd/4970

This Dissertation is protected by copyright and/or related rights. It has been brought to you by the The Research Repository @ WVU with permission from the rights-holder(s). You are free to use this Dissertation in any way that is permitted by the copyright and related rights legislation that applies to your use. For other uses you must obtain permission from the rights-holder(s) directly, unless additional rights are indicated by a Creative Commons license in the record and/ or on the work itself. This Dissertation has been accepted for inclusion in WVU Graduate Theses, Dissertations, and Problem Reports collection by an authorized administrator of The Research Repository @ WVU.

For more information, please contact researchrepository@mail.wvu.edu. 


\title{
Connectivity and spanning trees of graphs
}

\author{
Xiaofeng Gu \\ Dissertation submitted to the \\ Eberly College of Arts and Sciences \\ at West Virginia University \\ in partial fulfillment of the requirements \\ for the degree of \\ Doctor of Philosophy \\ in \\ Mathematics \\ Hong-Jian Lai, Ph.D., Chair \\ John Goldwasser, Ph.D. \\ Leonardo Golubovic, Ph.D. \\ Jerzy Wojciechowski, Ph.D. \\ Cun-Quan Zhang, Ph.D. \\ Department of Mathematics \\ West Virginia University \\ Morgantown, West Virginia 26506 \\ 2013
}

Keywords: Connectivity; Edge connectivity; Generalized connectivity; Spanning tree; Graph; Hypergraph 


\section{ABSTRACT \\ Connectivity and spanning trees of graphs \\ Xiaofeng Gu}

This dissertation focuses on connectivity, edge connectivity and edge-disjoint spanning trees in graphs and hypergraphs from the following aspects.

1. Eigenvalue aspect.

Let $\lambda_{2}(G)$ and $\tau(G)$ denote the second largest eigenvalue and the maximum number of edgedisjoint spanning trees of a graph $G$, respectively. Motivated by a question of Seymour on the relationship between eigenvalues of a graph $G$ and bounds of $\tau(G)$, Cioabă and Wong conjectured that for any integers $d, k \geq 2$ and a $d$-regular graph $G$, if $\lambda_{2}(G)<d-\frac{2 k-1}{d+1}$, then $\tau(G) \geq k$. They proved the conjecture for $k=2,3$, and presented evidence for the cases when $k \geq 4$. We propose a more general conjecture that for a graph $G$ with minimum degree $\delta \geq 2 k \geq 4$, if $\lambda_{2}(G)<\delta-\frac{2 k-1}{\delta+1}$, then $\tau(G) \geq k$. We prove the conjecture for $k=2,3$ and provide partial results for $k \geq 4$. We also prove that for a graph $G$ with minimum degree $\delta \geq k \geq 2$, if $\lambda_{2}(G)<\delta-\frac{2(k-1)}{\delta+1}$, then the edge connectivity is at least $k$. As corollaries, we investigate the Laplacian and signless Laplacian eigenvalue conditions on $\tau(G)$ and edge connectivity.

2. Network reliability aspect.

With graphs considered as natural models for many network design problems, edge connectivity $\kappa^{\prime}(G)$ and maximum number of edge-disjoint spanning trees $\tau(G)$ of a graph $G$ have been used as measures for reliability and strength in communication networks modeled as graph $G$. Let $\overline{\kappa^{\prime}}(G)=\max \left\{\kappa^{\prime}(H): H\right.$ is a subgraph of $\left.G\right\}$. We present:

(i) For each integer $k>0$, a characterization for graphs $G$ with the property that $\overline{\kappa^{\prime}}(G) \leq k$ but for any additional edge $e$ not in $G, \overline{\kappa^{\prime}}(G+e) \geq k+1$.

(ii) For any integer $n>0$, a characterization for graphs $G$ with $|V(G)|=n$ such that $\kappa^{\prime}(G)=$ $\tau(G)$ with $|E(G)|$ minimized.

\section{Generalized connectivity.}

For an integer $l \geq 2$, the $l$-connectivity $\kappa_{l}(G)$ of a graph $G$ is defined to be the minimum number of vertices of $G$ whose removal produces a disconnected graph with at least $l$ components or a graph with fewer than $l$ vertices. Let $k \geq 1$, a graph $G$ is called $(k, l)$-connected if $\kappa_{l}(G) \geq k$. A graph $G$ is called minimally $(k, l)$-connected if $\kappa_{l}(G) \geq k$ but $\forall e \in E(G), \kappa_{l}(G-e) \leq k-1$. A structural characterization for minimally $(2, l)$-connected graphs and some extremal results are obtained. These extend former results by Dirac and Plummer on minimally 2-connected graphs. 
4. Degree sequence aspect.

An integral sequence $d=\left(d_{1}, d_{2}, \cdots, d_{n}\right)$ is hypergraphic if there is a simple hypergraph $H$ with degree sequence $d$, and such a hypergraph $H$ is a realization of $d$. A sequence $d$ is $r$-uniform hypergraphic if there is a simple $r$-uniform hypergraph with degree sequence $d$. It is proved that an $r$-uniform hypergraphic sequence $d=\left(d_{1}, d_{2}, \cdots, d_{n}\right)$ has a $k$-edge-connected realization if and only if both $d_{i} \geq k$ for $i=1,2, \cdots, n$ and $\sum_{i=1}^{n} d_{i} \geq \frac{r(n-1)}{r-1}$, which generalizes the formal result of Edmonds for graphs and that of Boonyasombat for hypergraphs.

5. Partition connectivity augmentation and preservation.

Let $k$ be a positive integer. A hypergraph $H$ is $k$-partition-connected if for every partition $P$ of $V(H)$, there are at least $k(|P|-1)$ hyperedges intersecting at least two classes of $P$. We determine the minimum number of hyperedges in a hypergraph whose addition makes the resulting hypergraph $k$-partition-connected. We also characterize the hyperedges of a $k$-partition-connected hypergraph whose removal will preserve $k$-partition-connectedness. 


\section{Acknowledgements}

First and foremost, I am most indebted to my supervisor, Dr. Hong-Jian Lai, for his continued encouragement and support over these last few years. It is a pleasure to work under his supervision. Without him, this paper could not have come about.

I would also like to thank my other committee members: Dr. John Goldwasser, Dr. Leonardo Golubovic, Dr. Jerzy Wojciechowski, and Dr. Cun-Quan Zhang, for their help during my studies.

And finally, I would like to thank the Department of Mathematics and Eberly College of Arts and Sciences at West Virginia University for providing me with an excellent study environment and support during my study as a graduate student. 


\section{DEDICATION}

To

my father Chengqin Gu, my mother Qingyun Song, my wife Senmei Yao

and

my lovely daughter Xiyao Gu 


\section{Contents}

1 Preliminaries $\quad 1$

1.1 Notation and Terminology . . . . . . . . . . . . . . . . . . 1

1.2 Main results . . . . . . . . . . . . . . . . . . . 3

2 Spanning trees, edge connectivity and eigenvalues of graphs 6

2.1 Introduction . . . . . . . . . . . . . . . . . . . . . 6

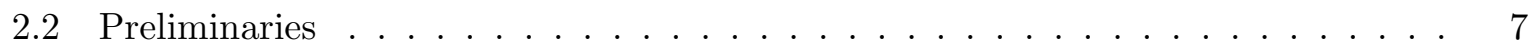

2.3 Eigenvalues and edge connectivity in graphs . . . . . . . . . . . . . . 9

2.4 Eigenvalues and edge-disjoint spanning trees . . . . . . . . . . . . . . 10

2.5 Laplacian and signless Laplacian eigenvalue conditions . . . . . . . . . . . . . 16

3 Strength extremal graphs $\quad 18$

3.1 Introduction . . . . . . . . . . . . . . . . . . . . . . . 18

3.2 Characterizations of $k$-maximal graphs . . . . . . . . . . . . . . . . 19

3.3 Characterizations of minimal graphs with $\kappa^{\prime}=\tau \ldots \ldots \ldots \ldots . \ldots 22$

3.4 Extensions and restrictions with respect to $\mathcal{F}_{k, n} \ldots \ldots \ldots \ldots \ldots$

4 Minimally $(2, l)$-connected graphs $\quad \mathbf{2 5}$

4.1 Introduction . . . . . . . . . . . . . . . . . . . . 25

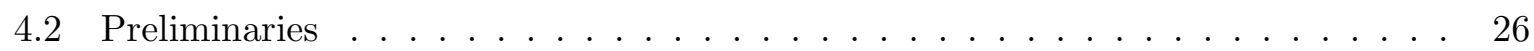

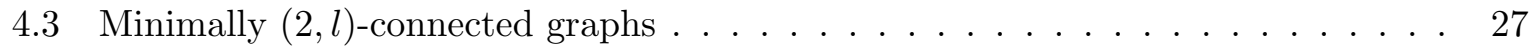

$4.4 F(n, 2, l), f(n, 2, l), \operatorname{Ex}(n, 2, l), \operatorname{Sat}(n, 2, l)$ and $\mathscr{I}(n, 2, l) \ldots \ldots \ldots$

$5 \quad$ Degree sequences and $k$-edge-connected uniform hypergraphs $\quad 35$

5.1 Introduction . . . . . . . . . . . . . . . . . . . . . . 35

5.2 The Proof of Theorem $5.1 .4 \ldots \ldots \ldots \ldots \ldots \ldots \ldots$

5.3 The Proof of Theorem $5.1 .5 \ldots \ldots \ldots \ldots \ldots \ldots \ldots$

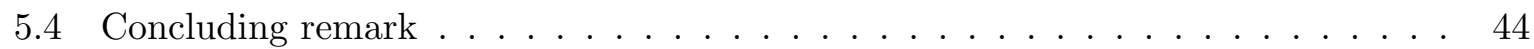


6 Augmenting and preserving partition connectivity of a hypergraph

6.1 The Problem . . . . . . . . . . . . . . . . . . . . . . 45

6.2 Notations and Preliminaries . . . . . . . . . . . . . . . . . . 46

6.3 Uniformly Dense Hypergraphs . . . . . . . . . . . . . . . . . . . . . . . . . 47

6.4 Complete Families and Decomposition Theorems . . . . . . . . . . . . . 50

6.5 Augmenting Partition Connectivity of a Hypergraph . . . . . . . . . . . . . 55

6.6 Preserving Partition Connectivity of a Hypergraph . . . . . . . . . . . . . . . 58 


\section{Chapter 1}

\section{Preliminaries}

\subsection{Notation and Terminology}

We follow notations of Bondy and Murty [6] for graphs and Berge [1] for hypergraphs, unless otherwise defined. Thus for a graph $G, \omega(G)$ denotes the number of components of $G$, and $\kappa^{\prime}(G)$ denotes the edge connectivity of $G$. A graph $G$ is nontrivial if $E(G) \neq \emptyset$. For a connected graph $G, \tau(G)$ denotes the maximum number of edge-disjoint spanning trees in $G$. A survey on $\tau(G)$ can be found in [63]. By definition, $\tau\left(K_{1}\right)=\infty$.

A fundamental theorem of Nash-Williams and Tutte characterizes graphs with at least $k$ edge-disjoint spanning trees.

Theorem 1.1.1. (Nash-Williams [57] and Tutte [70])

Let $G$ be a connected graph with $E(G) \neq \emptyset$, and let $k>0$ be an integer. Then $\tau(G) \geq k$ if and only if for any $X \subseteq E(G),|X| \geq k(\omega(G-X)-1)$.

Nash-Williams published a dual theorem of Theorem 1.1.1, characterizing graphs that can be decomposed to at most $k$ forests (Theorem 1.1.2).

Theorem 1.1.2. (Nash-Williams [58]) Let $G$ be a connected graph and $k$ be a positive integer. Then $a(G) \leq k$ if and only if for any subgraph $S,|E(S)| \leq k(|V(S)|-1)$.

Let $G$ be a graph. The density of $G$ is defined by

$$
d(G)=\frac{|E(G)|}{|V(G)|-\omega(G)} .
$$

Hence, if $G$ is connected, then $d(G)=\frac{|E(G)|}{|V(G)|-1}$. Following the terminology in [11], $\eta(G)$ and $\gamma(G)$ are respectively defined as

$$
\eta(G)=\min \frac{|X|}{\omega(G-X)-\omega(G)} \text { and } \gamma(G)=\max \{d(H)\}
$$


where the minimum or maximum is taken over all edge subsets $X$ or subgraph $H$ whenever the denominator is non-zero. From the definitions of $d(G), \eta(G)$ and $\gamma(G)$, we immediately have, for any nontrivial graph $G$,

$$
\eta(G) \leq d(G) \leq \gamma(G)
$$

As in [11], a graph $G$ satisfying $d(G)=\gamma(G)$ is said to be uniformly dense.

Theorem 1.1.1 above indicates that for a connected graph $G$

$$
\tau(G)=\lfloor\eta(G)\rfloor
$$

Theorem 1.1.3. (Catlin et al. [11])

Let $G$ be a graph. The following statements are equivalent.

(i) $\eta(G)=d(G)$.

(ii) $d(G)=\gamma(G)$.

(iii) $\eta(G)=\gamma(G)$.

Let $G$ be an undirected graph on $n$ vertices with vertex set $\left\{v_{1}, v_{2}, \cdots, v_{n}\right\}$. The adjacency matrix of $G$ is an $n$ by $n$ matrix $A(G)=\left(a_{i j}\right)$ given by $a_{i j}=m\left(v_{i}, v_{j}\right)$ where $m\left(v_{i}, v_{j}\right)$ denotes the number of edges between $v_{i}$ and $v_{j}$ for $1 \leq i, j \leq n$. By the definition, if $G$ is simple, then $A(G)$ is a symmetric $(0,1)$-matrix. Eigenvalues of $G$ are the eigenvalues of $A(G)$. We use $\lambda_{i}(G)$ to denote the $i$ th largest eigenvalue of $G$; and when the graph $G$ is understood from the context, we often use $\lambda_{i}$ for $\lambda_{i}(G)$. With these notations, we always have $\lambda_{1} \geq \lambda_{2} \geq \cdots \geq \lambda_{n}$.

Let $A(G)$ be the adjacency matrix of a graph $G$ and $D(G)$ be the diagonal matrix of row sums of $A(G)$ (i.e., the degrees of $G$ ), which is the degree matrix of $G$. The matrices $L(G)=$ $D(G)-A(G)$ and $Q(G)=D(G)+A(G)$ are the Laplacian matrix and the signless Laplacian matrix of $G$, respectively. We use $\mu_{i}(G)$ and $q_{i}(G)$ to denote the $i$ th largest eigenvalue of $L(G)$ and $Q(G)$, respectively. It is not difficult to see that $\mu_{n}(G)=0$. The second smallest eigenvalue of $L(G), \mu_{n-1}(G)$, is known as the algebraic connectivity of $G$.

A hypergraph $H$ is a pair $(V, \mathcal{E})$ where $V$ is the vertex set of $H$ and $\mathcal{E}$ is a collection of not necessarily distinct nonempty subsets of $V$. Note that we allow a hypergraph to have isolated vertices, which differs slightly from [1]. An element in $V$ is a vertex of $H$, and an element in $\mathcal{E}$ is a hyperedge or simply an edge of $H$. A hypergraph is nontrivial if $\mathcal{E} \neq \emptyset$. A single element edge is referred as a loop and two edges with the same vertices are parallel edges. We use $K_{1}$ to denote the hypergraph with one vertex and no edges. If $W \subset V$, the hypergraph $\left(W, \mathcal{E}_{W}\right)$, where $\mathcal{E}_{W}=\{F \cap W: \forall F \in \mathcal{E}$ with $F \cap W \neq \emptyset\}$, is a sub-hypergraph induced by the vertex subset $W$, and is denoted by $H[W]$. If $X \subseteq \mathcal{E}$ and $V_{X}=\cup_{F \in X} X$, then $\left(V_{X}, X\right)$ is defined as the sub-hypergraph induced by the edge subset $X$ and is denoted by $H[X]$. A hypergraph $H$ is nontrivial if $H$ has at least one non loop edge. Let $\omega(H)$ denote the number 
of components in $H$. The degree of a vertex $v$ in $H$, denoted by $d_{H}(v)$ or $d(v)$, is the number of edges in $H$ containing $v$. Let $\mathcal{E}=\left\{E_{1}, E_{2}, \cdots, E_{m}\right\}$. A hypergraph $H$ is simple if $E_{i} \subseteq E_{j}$ implies that $i=j$ for any $i, j$ with $1 \leq i, j \leq m$. Let $r \geq 2$ be an integer. A hypergraph $H$ is an $r$-uniform hypergraph if $\left|E_{i}\right|=r$ for each $i$ with $1 \leq i \leq m$. Thus a simple graph is a simple 2-uniform hypergraph, and vice versa. Let $G$ and $H$ be hypergraphs with $V(G) \cap V(H)=\emptyset$. Then $G \cup H$ is the hypergraph with vertex set $V(G) \cup V(H)$ and edge set $\mathcal{E}(G) \cup \mathcal{E}(H)$. If $X$ is a collection of nonempty subsets of $V(H)$ and $X \cap \mathcal{E}(H)=\emptyset$, then $H+X$ is the hypergraph with vertex set $V(H)$ and edge set $\mathcal{E}(H) \cup X$.

Let $H$ be a hypergraph and $V_{1}, V_{2}, \cdots, V_{k}$ be subsets of $V(H)$. A hyperedge $E \in \mathcal{E}(H)$ is $\left(V_{1}, V_{2}, \cdots, V_{k}\right)$-crossing if $E \cap V_{i} \neq \emptyset$ for $1 \leq i \leq k$. If in addition, $E \subseteq \cup_{i=1}^{k} V_{i}$, then $E$ is exact$\left(V_{1}, V_{2}, \cdots, V_{k}\right)$-crossing. When $k=1, E$ is said to be $V_{1}$-crossing and exact- $V_{1}$-crossing, respectively. The set of all exact- $\left(V_{1}, V_{2}, \cdots, V_{k}\right)$-crossing edges of $H$ is denoted by $\mathcal{E}_{V_{1} V_{2} \cdots V_{k}}^{H}$. A walk in a hypergraph $H$ is a finite alternating sequence $W=\left(v_{0}, E_{1}, v_{1}, E_{2}, \cdots, E_{k}, v_{k}\right)$, where $v_{i}$ is a vertex for $i=0,1, \cdots, k$ and $E_{j}$ is an edge such that $v_{j-1}, v_{j} \in E_{j}$ for $j=1,2, \cdots, k$. A walk $W$ is a path if all the vertices $v_{i}$ for $i=0,1, \cdots, k$ and all the edges in $W$ are distinct. A hypergraph is connected if for each pair of distinct vertices there exists a path from one to the other. Let $X$ be a nonempty proper subset of $V$ and $\bar{X}=V-X$. The set of all $(X, \bar{X})$-crossing hyperedges of a hypergraph $H$ is an edge-cut of $H$ between $X$ and $\bar{X}$, denoted by $[X, \bar{X}]_{H}$, or $[X, \bar{X}]$. The number of hyperedges in $[X, \bar{X}]_{H}$ is denoted by $\left|[X, \bar{X}]_{H}\right|$ or $d_{H}(X)$.

For a positive integer $k$, a hypergraph $H$ is $k$-edge-connected if for every nonempty proper subset $U$ of $V(H)$, there are at least $k$ hyperedges intersecting both $U$ and $V(H) \backslash U$. The edge connectivity of $H$ is the maximum $k$ such that $H$ is $k$-edge-connected. A hypergraph $H$ is $k$-partition-connected if $e(P) \geq k(|P|-1)$ for every partition $P$ of $V(H)$, where $|P|$ denotes the number of classes in $P$, and $e(P)$ denotes the number of edges intersecting at least two classes of $P$. Equivalently, $H$ is $k$-partition-connected if for any subset $X \subseteq \mathcal{E}(H)$, $|X| \geq k(\omega(H-X)-1)$. As $P$ can be any partitions of $V(H)$ into two nonempty subsets, it follows by definition that every $k$-partition-connected hypergraph must be $k$-edge-connected. Often a 1-partition-connected hypergraph is also referred as a partition-connected hypergaph. Note that a graph is partition-connected if and only if it is connected. In general, partition-connected hypergraphs must be connected, but a connected hypergraph may not be partition-connected. The partition connectivity of $H$ is the maximum $k$ such that $H$ is $k$-partition-connected.

\subsection{Main results}

The main results in the dissertation are summarized as below.

1. Seymour proposed the following problem: to determine the relationship between the eigen- 
values and the maximum number of edge-disjoint spanning trees in a graph. Cioabă and Wong conjectured that for any integers $d, k \geq 2$ and a $d$-regular graph $G$, if $\lambda_{2}(G)<$ $d-\frac{2 k-1}{d+1}$, then $\tau(G) \geq k$. They proved the conjecture for $k=2,3$, and presented evidence for the cases when $k \geq 4$. Thus the conjecture remains open for $k \geq 4$. We propose a more general conjecture that for a graph $G$ with minimum degree $\delta \geq 2 k \geq 4$, if $\lambda_{2}(G)<\delta-\frac{2 k-1}{\delta+1}$, then $\tau(G) \geq k$. In this paper, we prove that for a graph $G$ with minimum degree $\delta$, each of the following holds.

(i) For $k \in\{2,3\}$, if $\delta \geq 2 k$ and $\lambda_{2}(G)<\delta-\frac{2 k-1}{\delta+1}$, then $\tau(G) \geq k$.

(ii) For $k \geq 4$, if $\delta \geq 2 k$ and $\lambda_{2}(G)<\delta-\frac{3 k-1}{\delta+1}$, then $\tau(G) \geq k$.

Our results sharpen theorems of Cioabă and Wong and give a partial solution to Cioabă and Wong's conjecture and Seymour's problem. We also prove that for a graph $G$ with minimum degree $\delta \geq k \geq 2$, if $\lambda_{2}(G)<\delta-\frac{2(k-1)}{\delta+1}$, then the edge connectivity is at least $k$, which generalizes a former result of Cioabă. As corollaries, we investigate the Laplacian and signless Laplacian eigenvalue conditions on $\tau(G)$ and edge connectivity.

2. With graphs considered as natural models for many network design problems, edge connectivity $\kappa^{\prime}(G)$ and maximum number of edge-disjoint spanning trees $\tau(G)$ of a graph $G$ have been used as measures for reliability and strength in communication networks modeled as graph $G$ (see [21,54], among others). Mader [52] and Matula [53] introduced the maximum subgraph edge connectivity $\overline{\kappa^{\prime}}(G)=\max \left\{\kappa^{\prime}(H): H\right.$ is a subgraph of $\left.G\right\}$, and also consider $\overline{\kappa^{\prime}}(G)$ reflecting the strength of the graph $G$ (see [54]). Motivated by their many useful applications in network design and by the established inequalities

$$
\overline{\kappa^{\prime}}(G) \geq \kappa^{\prime}(G) \geq \tau(G)
$$

we present the following:

(i) For each integer $k>0$, a characterization for graphs $G$ with the property that $\overline{\kappa^{\prime}}(G) \leq k$ but for any additional edge $e$ not in $G, \overline{\kappa^{\prime}}(G+e) \geq k+1$.

(ii) For any integer $n>0$, a characterization for graphs $G$ with $|V(G)|=n$ such that $\kappa^{\prime}(G)=\tau(G)$ with $|E(G)|$ minimized.

3. For an integer $l \geq 2$, the $l$-connectivity $\kappa_{l}(G)$ of a graph $G$ is defined to be the minimum number of vertices of $G$ whose removal produces a disconnected graph with at least $l$ components or a graph with fewer than $l$ vertices. Let $k \geq 1$, a graph $G$ is called $(k, l)$ connected if $\kappa_{l}(G) \geq k$. A graph $G$ is called minimally $(k, l)$-connected if $\kappa_{l}(G) \geq k$ but $\forall e \in E(G), \kappa_{l}(G-e) \leq k-1$. We present a structural characterization for minimally $(2, l)$ connected graphs and classify extremal results. These extend former results by Dirac [23] and Plummer [64] on minimally 2-connected graphs. 
4. An integral sequence $d=\left(d_{1}, d_{2}, \cdots, d_{n}\right)$ is hypergraphic if there is a simple hypergraph $H$ with degree sequence $d$, and such a hypergraph $H$ is a realization of $d$. A sequence $d$ is $r$-uniform hypergraphic if there is a simple $r$-uniform hypergraph with degree sequence $d$. Similarly, a sequence $d$ is $r$-uniform multi-hypergraphic if there is an $r$-uniform hypergraph (possibly with multiple edges) with degree sequence $d$. It is proved that an $r$-uniform hypergraphic sequence $d=\left(d_{1}, d_{2}, \cdots, d_{n}\right)$ has a $k$-edge-connected realization if and only if both $d_{i} \geq k$ for $i=1,2, \cdots, n$ and $\sum_{i=1}^{n} d_{i} \geq \frac{r(n-1)}{r-1}$, which generalizes the formal result of Edmonds for graphs and that of Boonyasombat for hypergraphs. It is also proved that a nonincreasing integral sequence $d=\left(d_{1}, d_{2}, \cdots, d_{n}\right)$ is the degree sequence of a $k$-edgeconnected $r$-uniform hypergraph (possibly with multiple edges) if and only if $\sum_{i=1}^{n} d_{i}$ is a multiple of $r, d_{n} \geq k$ and $\sum_{i=1}^{n} d_{i} \geq \max \left\{\frac{r(n-1)}{r-1}, r d_{1}\right\}$.

5. Let $k$ be a positive integer. A hypergraph $H$ is $k$-partition-connected if for every partition $P$ of $V(H)$, there are at least $k(|P|-1)$ hyperedges intersecting at least two classes of $P$. We determine the minimum number of hyperedges in a hypergraph whose addition makes the resulting hypergraph $k$-partition-connected. We also characterize the hyperedges of a $k$-partition-connected hypergraph whose removal will preserve $k$-partition-connectedness. 


\section{Chapter 2}

\section{Spanning trees, edge connectivity and eigenvalues of graphs}

\subsection{Introduction}

In this paper, we consider finite undirected simple graphs.

Seymour proposed the following problem on predicting $\tau(G)$ by means of the eigenvalues.

Problem 1. ([19]) Let $G$ be a connected graph. Determine the relationship between $\tau(G)$ and eigenvalues of $G$.

Motivated by this problem of Seymour, Cioabă and Wong proposed the following conjecture.

Conjecture 2.1.1. (Cioabă and Wong [19]) Let $k$ and $d$ be two integers with $d \geq 2 k \geq 4$. If $G$ is a d-regular graph with $\lambda_{2}(G)<d-\frac{2 k-1}{d+1}$, then $\tau(G) \geq k$.

Utilizing Theorem 1.1.1, Cioabă [17], Cioabă and Wong [19] proved a number of theorems in this direction, settling Conjecture 2.1.1 for the cases when $k \in\{2,3\}$ and obtaining partial results towards the conjecture for other values of $k$.

Theorem 2.1.1. (Cioabă, Theorem 1.3 in [17]) Let $k$ and $d$ be two integers with $d \geq k \geq 2$. If $G$ is a d-regular graph with $\lambda_{2}(G)<d-\frac{2(k-1)}{d+1}$, then $\kappa^{\prime}(G) \geq k$.

Theorem 2.1.2. (Cioabă and Wong, Theorem 1.1 in [19]) Let $d$ be an integer with $d \geq 4$. If $G$ is a d-regular graph with $\lambda_{2}(G)<d-\frac{3}{d+1}$, then $\tau(G) \geq 2$.

Theorem 2.1.3. (Cioabă and Wong, Theorem 1.2 in [19]) Let $d$ be an integer with $d \geq 6$. If $G$ is a d-regular graph with $\lambda_{2}(G)<d-\frac{5}{d+1}$, then $\tau(G) \geq 3$.

Theorem 2.1.4. (Cioabă and Wong [19]) Let $k$ and $d$ be two integers with $d \geq 2 k \geq 4$. If $G$ is a d-regular graph with $\lambda_{2}(G)<d-\frac{2(2 k-1)}{d+1}$, then $\tau(G) \geq k$. 
The main purpose of this paper is to continue the investigation between eigenvalues of a simple graph (not necessarily regular) and the number of edge-disjoint spanning trees. As suggested by Theorem 1.1.1, high edge connectivity also implies more edge-disjoint spanning trees packing in a graph (see [36] for an example), we also investigate the relationship between edge connectivity of a simple graph and its second largest eigenvalue. Firstly, we present a more general conjecture, stated below.

Conjecture 2.1.2. Let $k$ be an integer with $k \geq 2$ and $G$ be a graph with minimum degree $\delta \geq 2 k$. If $\lambda_{2}(G)<\delta-\frac{2 k-1}{\delta+1}$, then $\tau(G) \geq k$.

The following are the main results in this paper. Theorem 2.1.5 generalizes Theorem 2.1.1. While Theorems 2.1.6 (i) and (ii) settle two special cases of Conjecture 2.1.2, Theorem 2.1.6 (iii) sheds some light to support Conjecture 2.1.2. Theorem 2.1.6 generalizes Theorems 2.1.2, 2.1.3 and 2.1.4, provides further evidence to support Conjectures 2.1.1 and 2.1.2, and sharpens Theorem 2.1.4.

Theorem 2.1.5. Let $k$ be an integer with $k \geq 2$ and $G$ be a graph with minimum degree $\delta \geq k$. If $\lambda_{2}(G)<\delta-\frac{2(k-1)}{\delta+1}$, then $\kappa^{\prime}(G) \geq k$.

Theorem 2.1.6. Let $k \geq 2$ be an integer, $G$ be a graph with minimum degree $\delta$.

(i) If $\delta \geq 4$ and $\lambda_{2}(G)<\delta-\frac{3}{\delta+1}$, then $\tau(G) \geq 2$.

(ii) If $\delta \geq 6$ and $\lambda_{2}(G)<\delta-\frac{5}{\delta+1}$, then $\tau(G) \geq 3$.

(iii) For $k \geq 4$, if $\delta \geq 2 k$ and $\lambda_{2}(G)<\delta-\frac{3 k-1}{\delta+1}$, then $\tau(G) \geq k$.

As applications of Theorem 2.1.5 and Theorem 2.1.6, we investigate the relationship between algebraic connectivity, the second largest eigenvalue of signless Laplacian matrix and edge connectivity, the number of edge-disjoint spanning trees of a simple graph.

In Section 2, we display some preliminaries and mechanisms, including eigenvalue interlacing properties and quotient matrices. These will be applied in the proofs of the main results, to be presented in Section 3 and 4. As corollaries, Laplacian and signless Laplacian eigenvalue conditions on $\tau(G)$ and edge connectivity are presented in the last section.

\subsection{Preliminaries}

In this section, we present some of the preliminaries and former results to be used in our arguments. Throughout this section, $G$ always denotes a simple graph.

Let $\mathbb{E}^{n}=\left\{\left(x_{1}, x_{2}, \cdots, x_{n}\right)^{T} \mid \sum_{i=1}^{n} x_{i}=1\right.$ and $x_{i} \geq 0$ for $\left.i=1,2, \cdots, n\right\}$.

Theorem 2.2.1. (Page 17 in [55]) Let $A$ be an irreducible nonnegative $n \times n$ matrix with the largest eigenvalue $\lambda_{1}$. Then

$$
\lambda_{1}=\min _{x \in \mathbb{E}^{n}}\left\{\max _{x_{i} \neq 0} \frac{(A x)_{i}}{x_{i}}\right\}=\max _{x \in \mathbb{E}^{n}}\left\{\min _{x_{i} \neq 0} \frac{(A x)_{i}}{x_{i}}\right\} .
$$


Theorem 2.2.2. (Proposition 3.1.2 in [8]) Let $G$ be a graph with largest eigenvalue $\lambda_{1}$, maximum degree $\Delta$ and average degree $\bar{d}$. Then $\bar{d} \leq \lambda_{1} \leq \Delta$.

Given two real sequences $\theta_{1} \geq \theta_{2} \geq \cdots \theta_{n}$ and $\eta_{1} \geq \eta_{2} \geq \cdots \geq \eta_{m}$ with $n>m$, the second sequence is said to interlace the first one if $\theta_{i} \geq \eta_{i} \geq \theta_{n-m+i}$, for $i=1,2, \cdots, m$. When we say the eigenvalues of a matrix $B$ interlace the eigenvalues of a matrix $A$, it means the non-increasing eigenvalue sequence of $B$ interlaces that of $A$. The following interlace results are well-known, and can be found in many textbooks.

Theorem 2.2.3. (Corollary 2.2 in [38]. See also [8,31]) Let $A$ be a real symmetric matrix and $B$ be a principal submatrix of $A$. Then the eigenvalues of $B$ interlace the eigenvalues of $A$.

Corollary 2.2.4. ( $[19,38]$ ) If $H$ is an induced subgraph of $G$, then the eigenvalues of $H$ interlace the eigenvalues of $G$.

Let $S$ and $T$ be disjoint subsets of $V(G)$. We denote by $E(S, T)$ the set of edges each of which has one vertex in $S$ and the other vertex in $T$ and let $e(S, T)=|E(S, T)|$. The next useful lemma follows immediately from Theorem 2.2.2 and Corollary 2.2.4.

Lemma 2.2.5. ( [19]) Let $S$ and $T$ be disjoint subsets of $V(G)$ and $e(S, T)=0$. Then

$$
\lambda_{2}(G) \geq \lambda_{2}(G[S \cup T]) \geq \min \left\{\lambda_{1}(G[S]), \lambda_{1}(G[T])\right\} \geq \min \{\bar{d}(G[S]), \bar{d}(G[T])\},
$$

where $\bar{d}$ denotes the average degree of a graph.

Suppose that we partition $V(G)$ into $s$ non-empty subsets $V_{1}, V_{2}, \cdots, V_{s}$. We denote this partition by $\pi$. The quotient matrix $A_{\pi}(G)=A\left(V_{1}, V_{2}, \cdots, V_{s}\right)$ of $G$ with respect to $\pi$, is an $s$ by $s$ matrix $\left(b_{i j}\right)$ such that $b_{i j}$ is the average number of neighbors in $V_{j}$ of the vertices in $V_{i}$ for $1 \leq i, j \leq s$. If the partition $\pi$ is not specified, we often use $A_{s}$ to denote the quotient matrix. As $A_{s}$ is an $s$ by $s$ square real matrix, the following is well known from linear algebra (for example, see Page 289 in [68]).

$$
\lambda_{1}\left(A_{s}\right)+\lambda_{2}\left(A_{s}\right)+\cdots+\lambda_{s}\left(A_{s}\right)=\operatorname{tr}\left(A_{s}\right) .
$$

We denote the average degree of $V_{i}$ by $\bar{d}_{i}$ for $1 \leq i, j \leq s$. By the definition of the quotient matrix, the sum of all entries in the $i$ th row is exactly $\bar{d}_{i}$. Let $\Delta_{\pi}(G)=\max _{1 \leq i \leq s}\left\{\bar{d}_{i}\right\}$ and $\delta_{\pi}(G)=\min _{1 \leq i \leq s}\left\{\bar{d}_{i}\right\}$. The following theorem is an analogue of Theorem 2.2.2.

Theorem 2.2.6. Let $G$ be a connected graph and $\pi$ be a partition of $V(G)$. Then

$$
\delta_{\pi} \leq \lambda_{1}\left(A_{\pi}\right) \leq \Delta_{\pi}
$$


Proof: Suppose that the partition $\pi$ has $s$ parts. Let $x=\left(\frac{1}{s}, \frac{1}{s}, \cdots, \frac{1}{s}\right)^{T} \in \mathbb{E}^{s}$. By Theorem 2.2.1,

$$
\lambda_{1}\left(A_{\pi}\right) \leq \max _{1 \leq i \leq s} \frac{(A x)_{i}}{x_{i}}=\max _{1 \leq i \leq s} \frac{\frac{1}{s} \cdot \bar{d}_{i}}{\frac{1}{s}}=\max _{1 \leq i \leq s} \bar{d}_{i}=\Delta_{\pi} .
$$

Similarly, by Theorem 2.2.1,

$$
\lambda_{1}\left(A_{\pi}\right) \geq \min _{1 \leq i \leq s} \frac{(A x)_{i}}{x_{i}}=\min _{1 \leq i \leq s} \frac{\frac{1}{s} \cdot \bar{d}_{i}}{\frac{1}{s}}=\min _{1 \leq i \leq s} \bar{d}_{i}=\delta_{\pi} .
$$

Theorem 2.2.7. (Corollary 2.3 in [38]. See also [8,31]) Let $G$ be a graph. Eigenvalues of any quotient matrix of $G$ interlace the eigenvalues of $G$.

Lemma 2.2.8. Let $G$ be a graph with minimum degree $\delta$ and $U$ be a non-empty proper subset of $V(G)$. If $e(U, V \backslash U) \leq \delta-1$, then $|U| \geq \delta+1$.

Proof: We argue by contradiction and assume that $|U| \leq \delta$. Then $|U|(|U|-1)+e(U, V \backslash U) \geq$ $|U| \delta$ by counting the total degrees of vertices in $U$. But $|U|(|U|-1)+e(U, V \backslash U) \leq \delta(|U|-1)+$ $(\delta-1) \leq|U| \delta-1$, contrary to the fact that $|U|(|U|-1)+e(U, V \backslash U) \geq|U| \delta$. Thus $|U| \geq \delta+1$.

\subsection{Eigenvalues and edge connectivity in graphs}

In this section, we present the proof of Theorem 2.1.5.

Proof of Theorem 2.1.5. We argue by contradiction and assume that $\kappa^{\prime}(G) \leq k-1$. Then there exists a non-empty proper subset $V_{1} \subseteq V(G)$ such that $e\left(V_{1}, V \backslash V_{1}\right) \leq k-1$. Let $r=$ $e\left(V_{1}, V \backslash V_{1}\right)$ and $V^{\prime}=V \backslash V_{1}$. By Lemma 2.2.8, $\left|V_{1}\right| \geq \delta+1$ and $\left|V^{\prime}\right| \geq \delta+1$. The quotient matrix of $G$ with respect to the partition $\left(V_{1}, V^{\prime}\right)$ is

$$
A_{2}=\left[\begin{array}{cc}
\bar{d}_{1}-\frac{r}{\left|V_{1}\right|} & \frac{r}{\left|V_{1}\right|} \\
\frac{r}{\left|V^{\prime}\right|} & \bar{d}^{\prime}-\frac{r}{\left|V^{\prime}\right|}
\end{array}\right],
$$

where $\bar{d}_{1}$ denotes the average degree of $V_{1}$ in $G$ and $\bar{d}^{\prime}$ denotes the average degree of $V^{\prime}$ in G. By $(2.1), \lambda_{2}\left(A_{2}\right)=\operatorname{tr}\left(A_{2}\right)-\lambda_{1}\left(A_{2}\right)$. By Theorem 2.2.6, $\lambda_{1}\left(A_{2}\right) \leq \max \left\{\bar{d}_{1}, \bar{d}^{\prime}\right\}$ and by Theorem 2.2.7, $\lambda_{2}\left(A_{2}\right) \leq \lambda_{2}(G)$. Thus $\lambda_{2}(G) \geq \lambda_{2}\left(A_{2}\right) \geq \operatorname{tr}\left(A_{2}\right)-\max \left\{\bar{d}_{1}, \bar{d}^{\prime}\right\}$, which implies that

$$
\lambda_{2}(G) \geq \operatorname{tr}\left(A_{2}\right)-\max \left\{\bar{d}_{1}, \bar{d}^{\prime}\right\}=\bar{d}_{1}+\bar{d}^{\prime}-\left(\frac{r}{\left|V_{1}\right|}+\frac{r}{\left|V^{\prime}\right|}\right)-\max \left\{\bar{d}_{1}, \bar{d}^{\prime}\right\} \geq \delta-\frac{2(k-1)}{\delta+1},
$$

contrary to the fact that $\lambda_{2}(G)<\delta-\frac{2(k-1)}{\delta+1}$. This completes the proof of the theorem. 


\subsection{Eigenvalues and edge-disjoint spanning trees}

The proof for Theorem 2.1.6 will be given in this section. We shall argue by contradiction and assume that $\tau(G) \leq k-1$. By Theorem 1.1.1, there exists an edge subset $X \subseteq E(G)$ such that $|X| \leq k(\omega(G-X)-1)-1$. Let $\omega(G-X)=t$ and $G_{1}, G_{2} \cdots, G_{t}$ be the components of $G-X$. For $1 \leq i \leq t$, let $V_{i}=V\left(G_{i}\right), E_{i}=E\left(G_{i}\right)$, and $r_{i}=e\left(V_{i}, V \backslash V_{i}\right)$. Without lose of generality, we always assume that

$$
r_{1} \leq r_{2} \leq \cdots \leq r_{t}
$$

With these notations and by $|X| \leq k(\omega(G-X)-1)-1$, we have

$$
\sum_{1 \leq i<j \leq t} e\left(V_{i}, V_{j}\right) \leq k(t-1)-1=k t-k-1 .
$$

Claim 1. For $k \geq 2$, if $\lambda_{2}(G)<\delta-\frac{2 k-1}{\delta+1}$, then there exist no indices $p$ and $q$ with $1 \leq p \neq q \leq t$ such that $e\left(V_{p}, V_{q}\right)=0$ and $r_{p}, r_{q} \leq 2 k-1$.

By Lemma 2.2.8, $\left|V_{p}\right| \geq \delta+1$ and $\left|V_{q}\right| \geq \delta+1$. It follows that $\bar{d}\left(G_{p}\right) \geq \delta-\frac{2 k-1}{\left|V_{p}\right|} \geq \delta-\frac{2 k-1}{\delta+1}$ and $\bar{d}\left(G_{q}\right) \geq \delta-\frac{2 k-1}{\left|V_{q}\right|} \geq \delta-\frac{2 k-1}{\delta+1}$. By Lemma 2.2.5, $\lambda_{2}(G) \geq \min \left\{\bar{d}\left(G_{p}\right), \bar{d}\left(G_{q}\right)\right\} \geq \delta-\frac{2 k-1}{\delta+1}$, contrary to the assumption that $\lambda_{2}(G)<\delta-\frac{2 k-1}{\delta+1}$. Thus the proof for Claim 1 is done.

Claim 2. For $k \geq 2$, if $\delta \geq 2 k$ and if $\lambda_{2}(G)<\delta-\frac{2 k-1}{\delta+1}$, then for any $i$ with $1 \leq i \leq t, r_{i} \geq k$.

We argue by contradiction and assume that for some $i, r_{i}<k$. Then $\kappa^{\prime}(G)<k$. By Theorem 2.1.5, $\lambda_{2}(G) \geq \delta-\frac{2(k-1)}{\delta+1}$, contrary to the assumption that $\lambda_{2}(G)<\delta-\frac{2 k-1}{\delta+1}$. Therefore, we must have $r_{i} \geq k$. This proves Claim 2 .

\section{The case when $k=2$}

In this subsection, we shall prove Theorem 2.1.6(i). By (2.3) with $k=2$, we have

$$
\sum_{i=1}^{t} r_{i}=2 \sum_{1 \leq i<j \leq t} e\left(V_{i}, V_{j}\right) \leq 4 t-6 .
$$

Let $x_{l}$ denote the multiplicity of $l$ in $\left\{r_{1}, r_{2}, \cdots, r_{t}\right\}$ for $l=1,2,3$. By Claim $2, r_{t} \geq \cdots \geq$ $r_{2} \geq r_{1} \geq 2$. Thus $x_{1}=0$. It follows by (2.3) with $k=2$ that

$$
2 x_{2}+3 x_{3}+4\left(t-x_{2}-x_{3}\right) \leq \sum_{i=1}^{t} r_{i} \leq 4 t-6,
$$

which implies that $2 x_{2}+x_{3} \geq 6$. Thus if $x_{2}=0$, then $x_{3} \geq 6$; and if $x_{2}=1$, then $x_{3} \geq 4$. It follows that when $0 \leq x_{2} \leq 1$, there always exist $p$ and $q$ with $1 \leq p \neq q \leq t$ such that $e\left(V_{p}, V_{q}\right)=0$ and $r_{p} \leq 3$ and $r_{q}=3$. But such indices $p$ and $q$ are forbidden by Claim 1 , a 
contradiction.

Hence we must have $x_{2} \geq 2$, and so we may assume, by (2.2), that $r_{1}, r_{2}=2$ and $2 \leq r_{3} \leq 3$. Let $V^{\prime}=V \backslash\left(V_{1} \cup V_{2}\right)$. Then $V_{3} \subseteq V^{\prime}$. By Lemma 2.2.8, $\left|V_{i}\right| \geq \delta+1$ for $i=1,2,3$, and so $\left|V^{\prime}\right| \geq\left|V_{3}\right| \geq \delta+1$. The quotient matrix of $G$ with respect to the partition $\left(V_{1}, V_{2}, V^{\prime}\right)$ is

$$
A_{3}=\left[\begin{array}{ccc}
\bar{d}_{1}-\frac{2}{\left|V_{1}\right|} & \frac{1}{\left|V_{1}\right|} & \frac{1}{\left|V_{1}\right|} \\
\frac{1}{\left|V_{2}\right|} & \bar{d}_{2}-\frac{2}{\left|V_{2}\right|} & \frac{1}{\left|V_{2}\right|} \\
\frac{1}{\left|V^{\prime}\right|} & \frac{1}{\left|V^{\prime}\right|} & \overline{d^{\prime}}-\frac{2}{\left|V^{\prime}\right|}
\end{array}\right],
$$

where $\bar{d}_{i}$ denotes the average degree of $V_{i}$ in $G$ for $i=1,2$ and $\bar{d}^{\prime}$ denotes the average degree of $V^{\prime}$ in $G$.

By $(2.1), \lambda_{2}\left(A_{3}\right)+\lambda_{3}\left(A_{3}\right)=\operatorname{tr}\left(A_{3}\right)-\lambda_{1}\left(A_{3}\right)$. By Theorem 2.2.7, $\lambda_{2}(G) \geq \lambda_{2}\left(A_{3}\right), \lambda_{3}(G) \geq$ $\lambda_{3}\left(A_{3}\right)$ and by Theorem 2.2.6, $\lambda_{1}\left(A_{3}\right) \leq \max \left\{\bar{d}_{1}, \bar{d}_{2}, \bar{d}^{\prime}\right\}$. Thus $\lambda_{2}(G)+\lambda_{3}(G) \geq \operatorname{tr}\left(A_{3}\right)-$ $\max \left\{\bar{d}_{1}, \bar{d}_{2}, \bar{d}^{\prime}\right\}$. As $\lambda_{2}(G) \geq \lambda_{3}(G)$, we have

$2 \lambda_{2}(G) \geq \operatorname{tr}\left(A_{3}\right)-\max \left\{\bar{d}_{1}, \bar{d}_{2}, \bar{d}^{\prime}\right\}=\bar{d}_{1}+\bar{d}_{2}+\bar{d}^{\prime}-\left(\frac{2}{\left|V_{1}\right|}+\frac{2}{\left|V_{2}\right|}+\frac{2}{\left|V^{\prime}\right|}\right)-\max \left\{\bar{d}_{1}, \bar{d}_{2}, \bar{d}^{\prime}\right\} \geq 2 \delta-\frac{6}{\delta+1}$, contrary to the assumption in Theorem 2.1.6 (i) that $\lambda_{2}(G)<\delta-\frac{3}{\delta+1}$. This completes the proof of Theorem 2.1.6 (i).

\section{The case when $k=3$}

In this subsection, we shall prove Theorem 2.1.6(ii). By (2.3) with $k=3$, we have

$$
\sum_{i=1}^{t} r_{i}=2 \sum_{1 \leq i<j \leq t} e\left(V_{i}, V_{j}\right) \leq 6 t-8 .
$$

Let $x_{l}$ denote the multiplicity of $l$ in $\left\{r_{1}, r_{2}, \cdots, r_{t}\right\}$ for $1 \leq l \leq 5$. By Claim $2, r_{t} \geq \cdots \geq r_{2} \geq$ $r_{1} \geq 3$. Thus $x_{1}=x_{2}=0$. It follows that

$$
3 x_{3}+4 x_{4}+5 x_{5}+6\left(t-x_{3}-x_{4}-x_{5}\right) \leq \sum_{i=1}^{t} r_{i} \leq 6 t-8,
$$

which implies that $3 x_{3}+2 x_{4}+x_{5} \geq 8$.

\section{Case 1: $x_{3} \geq 2$.}

Then by (2.2), $r_{1}=r_{2}=3$ and $r_{3} \leq 5$. By Lemma 2.2.8, $\left|V_{i}\right| \geq \delta+1$ for $i=1,2,3$. Let $V^{\prime}=V \backslash\left(V_{1} \cup V_{2}\right)$. Then $\left|V^{\prime}\right| \geq\left|V_{3}\right| \geq \delta+1$. The quotient matrix of $G$ with respect to the partition $\left(V_{1}, V_{2}, V^{\prime}\right)$ is

$$
A_{3}=\left[\begin{array}{ccc}
\bar{d}_{1}-\frac{3}{\left|V_{1}\right|} & \frac{1}{\left|V_{1}\right|} & \frac{2}{\left|V_{1}\right|} \\
\frac{1}{\left|V_{2}\right|} & \bar{d}_{2}-\frac{3}{\left|V_{2}\right|} & \frac{2}{\left|V_{2}\right|} \\
\frac{2}{\left|V^{\prime}\right|} & \frac{2}{\left|V^{\prime}\right|} & \overline{d^{\prime}}-\frac{4}{\left|V^{\prime}\right|}
\end{array}\right]
$$


where $\bar{d}_{i}$ denotes the average degree of $V_{i}$ in $G$ for $i=1,2$ and $\bar{d}^{\prime}$ denotes the average degree of $V^{\prime}$ in $G$.

By $(2.1), \lambda_{2}\left(A_{3}\right)+\lambda_{3}\left(A_{3}\right)=\operatorname{tr}\left(A_{3}\right)-\lambda_{1}\left(A_{3}\right)$. By Theorem 2.2.7, $\lambda_{2}(G) \geq \lambda_{2}\left(A_{3}\right), \lambda_{3}(G) \geq$ $\lambda_{3}\left(A_{3}\right)$ and by Theorem 2.2.6, $\lambda_{1}\left(A_{3}\right) \leq \max \left\{\bar{d}_{1}, \bar{d}_{2}, \bar{d}^{\prime}\right\}$. Thus $\lambda_{2}(G)+\lambda_{3}(G) \geq \operatorname{tr}\left(A_{3}\right)-$ $\max \left\{\bar{d}_{1}, \bar{d}_{2}, \bar{d}^{\prime}\right\}$. As $\lambda_{2}(G) \geq \lambda_{3}(G)$, we have

$2 \lambda_{2}(G) \geq \operatorname{tr}\left(A_{3}\right)-\max \left\{\bar{d}_{1}, \bar{d}_{2}, \bar{d}^{\prime}\right\}=\bar{d}_{1}+\bar{d}_{2}+\bar{d}^{\prime}-\left(\frac{3}{\left|V_{1}\right|}+\frac{3}{\left|V_{2}\right|}+\frac{4}{\left|V^{\prime}\right|}\right)-\max \left\{\bar{d}_{1}, \bar{d}_{2}, \bar{d}^{\prime}\right\} \geq 2 \delta-\frac{10}{\delta+1}$,

contrary to the assumption in Theorem 2.1.6 (ii) that $\lambda_{2}(G)<\delta-\frac{5}{\delta+1}$.

Case 2: $x_{3}=1$.

Hence $2 x_{4}+x_{5} \geq 5$. If $x_{4}=0$, then $x_{5} \geq 5$, and so there exist $p$ and $q$ with $1 \leq p \neq q \leq t$ such that $e\left(V_{p}, V_{q}\right)=0$ and $r_{p}=3$ and $r_{q}=5$. This is prohibited by Claim 2. Therefore we must have $x_{4} \geq 1$, and so by $(2.2), r_{1}=3, r_{2}=4$, and $r_{3}, r_{4} \leq 5$. By Lemma $2.2 .8,\left|V_{i}\right| \geq \delta+1$ for $i=1,2,3,4$. Let $V^{\prime}=V \backslash\left(V_{1} \cup V_{2}\right)$. Thus $V_{3}, V_{4} \subseteq V^{\prime}$, whence $\left|V^{\prime}\right| \geq 2(\delta+1)$. The quotient matrix of $G$ with respect to the partition $\left(V_{1}, V_{2}, V^{\prime}\right)$ is

$$
A_{3}=\left[\begin{array}{ccc}
\bar{d}_{1}-\frac{3}{\left|V_{1}\right|} & \frac{1}{\left|V_{1}\right|} & \frac{2}{\left|V_{1}\right|} \\
\frac{1}{\left|V_{2}\right|} & \bar{d}_{2}-\frac{4}{\left|V_{2}\right|} & \frac{3}{\left|V_{2}\right|} \\
\frac{2}{\left|V^{\prime}\right|} & \frac{3}{\left|V^{\prime}\right|} & \overline{d^{\prime}}-\frac{5}{\left|V^{\prime}\right|}
\end{array}\right],
$$

where $\bar{d}_{i}$ denotes the average degree of $V_{i}$ in $G$ for $i=1,2$ and $\bar{d}^{\prime}$ denotes the average degree of $V^{\prime}$ in $G$.

By $(2.1), \lambda_{2}\left(A_{3}\right)+\lambda_{3}\left(A_{3}\right)=\operatorname{tr}\left(A_{3}\right)-\lambda_{1}\left(A_{3}\right)$. By Theorem 2.2.7, $\lambda_{2}(G) \geq \lambda_{2}\left(A_{3}\right), \lambda_{3}(G) \geq$ $\lambda_{3}\left(A_{3}\right)$ and by Theorem 2.2.6, $\lambda_{1}\left(A_{3}\right) \leq \max \left\{\bar{d}_{1}, \bar{d}_{2}, \bar{d}^{\prime}\right\}$. Thus $\lambda_{2}(G)+\lambda_{3}(G) \geq \operatorname{tr}\left(A_{3}\right)-$ $\max \left\{\bar{d}_{1}, \bar{d}_{2}, \bar{d}^{\prime}\right\}$. As $\lambda_{2}(G) \geq \lambda_{3}(G)$, we have

$2 \lambda_{2}(G) \geq \operatorname{tr}\left(A_{3}\right)-\max \left\{\bar{d}_{1}, \bar{d}_{2}, \bar{d}^{\prime}\right\}=\bar{d}_{1}+\bar{d}_{2}+\bar{d}^{\prime}-\left(\frac{3}{\left|V_{1}\right|}+\frac{4}{\left|V_{2}\right|}+\frac{5}{\left|V^{\prime}\right|}\right)-\max \left\{\bar{d}_{1}, \bar{d}_{2}, \bar{d}^{\prime}\right\} \geq 2 \delta-\frac{19 / 2}{\delta+1}$,

contrary to the assumption in Theorem 2.1.6 (ii) that $\lambda_{2}(G)<\delta-\frac{5}{\delta+1}$.

Case 3: $x_{3}=0$.

Then $2 x_{4}+x_{5} \geq 8$. If $x_{4}<2$, then either $x_{4}=1$ and $x_{5} \geq 6$, or $x_{4}=0$ and $x_{5} \geq 8$. In either case, there exist $p$ and $q$ with $1 \leq p \neq q \leq t$ such that $e\left(V_{p}, V_{q}\right)=0$ and $r_{p}, r_{q} \leq 5$, violating Claim 2. Hence, by (2.2), we may assume that $r_{1}=r_{2}=4$. Since $2 x_{4}+x_{5} \geq 8, r_{3}, r_{4} \leq 5$.

Case 3.1. $r_{5} \leq 5$.

Let $V^{\prime}=V \backslash\left(V_{1} \cup V_{2}\right)$. By Lemma 2.2.8, $\left|V_{i}\right| \geq \delta+1$ for $i=1,2,3,4,5$. Then $\left|V^{\prime}\right| \geq$ $\left|V_{3}\right|+\left|V_{4}\right|+\left|V_{5}\right| \geq 3(\delta+1)$. The quotient matrix of $G$ with respect to the partition $\left(V_{1}, V_{2}, V^{\prime}\right)$ 
is

$$
A_{3}=\left[\begin{array}{ccc}
\bar{d}_{1}-\frac{4}{\left|V_{1}\right|} & \frac{1}{\left|V_{1}\right|} & \frac{3}{\left|V_{1}\right|} \\
\frac{1}{\left|V_{2}\right|} & \bar{d}_{2}-\frac{4}{\left|V_{2}\right|} & \frac{3}{\left|V_{2}\right|} \\
\frac{3}{\left|V^{\prime}\right|} & \frac{3}{\left|V^{\prime}\right|} & \bar{d}^{\prime}-\frac{6}{\left|V^{\prime}\right|}
\end{array}\right],
$$

where $\bar{d}_{i}$ denotes the average degree of $V_{i}$ in $G$ for $i=1,2$ and $\bar{d}^{\prime}$ denotes the average degree of $V^{\prime}$ in $G$.

By $(2.1), \lambda_{2}\left(A_{3}\right)+\lambda_{3}\left(A_{3}\right)=\operatorname{tr}\left(A_{3}\right)-\lambda_{1}\left(A_{3}\right)$. By Theorem 2.2.7, $\lambda_{2}(G) \geq \lambda_{2}\left(A_{3}\right), \lambda_{3}(G) \geq$ $\lambda_{3}\left(A_{3}\right)$ and by Theorem 2.2.6, $\lambda_{1}\left(A_{3}\right) \leq \max \left\{\bar{d}_{1}, \bar{d}_{2}, \bar{d}^{\prime}\right\}$. Thus $\lambda_{2}(G)+\lambda_{3}(G) \geq \operatorname{tr}\left(A_{3}\right)-$ $\max \left\{\bar{d}_{1}, \bar{d}_{2}, \bar{d}^{\prime}\right\}$. As $\lambda_{2}(G) \geq \lambda_{3}(G)$, we have

$2 \lambda_{2}(G) \geq \operatorname{tr}\left(A_{3}\right)-\max \left\{\bar{d}_{1}, \bar{d}_{2}, \bar{d}^{\prime}\right\}=\bar{d}_{1}+\bar{d}_{2}+\bar{d}^{\prime}-\left(\frac{4}{\left|V_{1}\right|}+\frac{4}{\left|V_{2}\right|}+\frac{6}{\left|V^{\prime}\right|}\right)-\max \left\{\bar{d}_{1}, \bar{d}_{2}, \bar{d}^{\prime}\right\} \geq 2 \delta-\frac{10}{\delta+1}$,

contrary to the assumption in Theorem 2.1.6 (ii) that $\lambda_{2}(G)<\delta-\frac{5}{\delta+1}$.

Case 3.2. $r_{5}>5$.

As $2 x_{4}+x_{5} \geq 8$, we must have $r_{i}=4$ for $i=1,2,3,4$. If $t=4$, then $\left(V_{1}, V_{2}, V_{3}, V_{4}\right)$ is a partition of $V(G)$. By Claim 2, and since $r_{1}=4$, there exists $V_{j}$ (say $j=2$ ) such that $e\left(V_{1}, V_{j}\right)=2$. Let $V^{\prime}=V \backslash\left(V_{1} \cup V_{2}\right)$. Then $\left|V^{\prime}\right|=\left|V_{3}\right|+\left|V_{4}\right| \geq 2(\delta+1)$. The quotient matrix of $G$ with respect to the partition $\left(V_{1}, V_{2}, V^{\prime}\right)$ is

$$
A_{3}=\left[\begin{array}{ccc}
\bar{d}_{1}-\frac{4}{\left|V_{1}\right|} & \frac{2}{\left|V_{1}\right|} & \frac{2}{\left|V_{1}\right|} \\
\frac{2}{\left|V_{2}\right|} & \bar{d}_{2}-\frac{4}{\left|V_{2}\right|} & \frac{2}{\left|V_{2}\right|} \\
\frac{2}{\left|V^{\prime}\right|} & \frac{2}{\left|V^{\prime}\right|} & \overline{d^{\prime}}-\frac{4}{\left|V^{\prime}\right|}
\end{array}\right],
$$

where $\bar{d}_{i}$ denotes the average degree of $V_{i}$ in $G$ for $i=1,2$ and $\bar{d}^{\prime}$ denotes the average degree of $V^{\prime}$ in $G$.

By $(2.1), \lambda_{2}\left(A_{3}\right)+\lambda_{3}\left(A_{3}\right)=\operatorname{tr}\left(A_{3}\right)-\lambda_{1}\left(A_{3}\right)$. By Theorem 2.2.7, $\lambda_{2}(G) \geq \lambda_{2}\left(A_{3}\right), \lambda_{3}(G) \geq$ $\lambda_{3}\left(A_{3}\right)$ and by Theorem 2.2.6, $\lambda_{1}\left(A_{3}\right) \leq \max \left\{\bar{d}_{1}, \bar{d}_{2}, \bar{d}^{\prime}\right\}$. Thus $\lambda_{2}(G)+\lambda_{3}(G) \geq \operatorname{tr}\left(A_{3}\right)-$ $\max \left\{\bar{d}_{1}, \bar{d}_{2}, \bar{d}^{\prime}\right\}$. As $\lambda_{2}(G) \geq \lambda_{3}(G)$, we have

$2 \lambda_{2}(G) \geq \operatorname{tr}\left(A_{3}\right)-\max \left\{\bar{d}_{1}, \bar{d}_{2}, \bar{d}^{\prime}\right\}=\bar{d}_{1}+\bar{d}_{2}+\bar{d}^{\prime}-\left(\frac{4}{\left|V_{1}\right|}+\frac{4}{\left|V_{2}\right|}+\frac{4}{\left|V^{\prime}\right|}\right)-\max \left\{\bar{d}_{1}, \bar{d}_{2}, \bar{d}^{\prime}\right\} \geq 2 \delta-\frac{10}{\delta+1}$,

contrary to the assumption in Theorem 2.1.6 (ii) that $\lambda_{2}(G)<\delta-\frac{5}{\delta+1}$.

If $t \geq 5$, then let $V^{\prime \prime}=V \backslash\left(V_{1} \cup V_{2} \cup V_{3} \cup V_{4}\right)$, and so $\left(V_{1}, V_{2}, V_{3}, V_{4}, V^{\prime \prime}\right)$ is a partition of $V(G)$. By Claim 2, we may assume that $e\left(V_{i}, V_{j}\right) \geq 1$ for $1 \leq i, j \leq 4$. Then $e\left(V^{\prime \prime}, V \backslash V^{\prime \prime}\right) \leq 4 \leq \delta-1$. By Lemma 2.2.8, $\left|V^{\prime \prime}\right| \geq \delta+1$. Let $V^{\prime}=V \backslash\left(V_{1} \cup V_{2}\right)$. Then $\left|V^{\prime}\right|=\left|V_{3}\right|+\left|V_{4}\right|+\left|V^{\prime \prime}\right| \geq 3(\delta+1)$. Let $e\left(V_{1}, V_{2}\right)=y$. Then $y \geq 1$. The quotient matrix of $G$ with respect to the partition $\left(V_{1}, V_{2}, V^{\prime}\right)$ is

$$
A_{3}=\left[\begin{array}{ccc}
\bar{d}_{1}-\frac{4}{\left|V_{1}\right|} & \frac{y}{\left|V_{1}\right|} & \frac{4-y}{\left|V_{1}\right|} \\
\frac{y}{\left|V_{2}\right|} & \bar{d}_{2}-\frac{4}{\left|V_{2}\right|} & \frac{4-y}{\left|V_{2}\right|} \\
\frac{4-y}{\left|V^{\prime}\right|} & \frac{4-y}{\left|V^{\prime}\right|} & \bar{d}^{\prime}-\frac{2(4-y)}{\left|V^{\prime}\right|}
\end{array}\right]
$$


where $\bar{d}_{i}$ denotes the average degree of $V_{i}$ in $G$ for $i=1,2$ and $\bar{d}^{\prime}$ denotes the average degree of $V^{\prime}$ in $G$.

By $(2.1), \lambda_{2}\left(A_{3}\right)+\lambda_{3}\left(A_{3}\right)=\operatorname{tr}\left(A_{3}\right)-\lambda_{1}\left(A_{3}\right)$. By Theorem 2.2.7, $\lambda_{2}(G) \geq \lambda_{2}\left(A_{3}\right), \lambda_{3}(G) \geq$ $\lambda_{3}\left(A_{3}\right)$ and by Theorem 2.2.6, $\lambda_{1}\left(A_{3}\right) \leq \max \left\{\bar{d}_{1}, \bar{d}_{2}, \bar{d}^{\prime}\right\}$. Thus $\lambda_{2}(G)+\lambda_{3}(G) \geq \operatorname{tr}\left(A_{3}\right)-$ $\max \left\{\bar{d}_{1}, \bar{d}_{2}, \bar{d}^{\prime}\right\}$. As $\lambda_{2}(G) \geq \lambda_{3}(G)$, we have

$$
\begin{aligned}
2 \lambda_{2}(G) & \geq \operatorname{tr}\left(A_{3}\right)-\max \left\{\bar{d}_{1}, \bar{d}_{2}, \bar{d}^{\prime}\right\} \\
& =\bar{d}_{1}+\bar{d}_{2}+\bar{d}^{\prime}-\left(\frac{4}{\left|V_{1}\right|}+\frac{4}{\left|V_{2}\right|}+\frac{2(4-y)}{\left|V^{\prime}\right|}\right)-\max \left\{\bar{d}_{1}, \bar{d}_{2}, \bar{d}^{\prime}\right\} \\
& \geq 2 \delta-\frac{10}{\delta+1},
\end{aligned}
$$

contrary to the assumption in Theorem 2.1.6 (ii) that $\lambda_{2}(G)<\delta-\frac{5}{\delta+1}$. This completes the proof.

\section{The case when $k \geq 4$}

In this subsection, we shall prove Theorem 2.1.6(iii). Let $x_{l}$ denote the multiplicity of $l$ in $\left\{r_{1}, r_{2}, \cdots, r_{t}\right\}$ for $1 \leq l \leq 2 k-1$. By Claim $2, r_{t} \geq \cdots \geq r_{2} \geq r_{1} \geq k$. Thus $x_{j}=0$ for $j=1,2, \cdots, k-1$. By $(2.3)$, we have

$k x_{k}+(k+1) x_{k+1}+\cdots+(2 k-1) x_{2 k-1}+2 k\left(t-\left(x_{k}+x_{k+1}+\cdots+x_{2 k-1}\right)\right) \leq \sum_{i=1}^{t} r_{i} \leq 2 k t-2(k+1)$,

which implies that

$$
k x_{k}+(k-1) x_{k+1}+\cdots+2 x_{2 k-2}+x_{2 k-1} \geq 2(k+1) .
$$

Let $h$ be the smallest index such that $x_{h} \neq 0$. Then we have

$$
(2 k-h) x_{h}+(2 k-h-1) x_{h+1}+\cdots+2 x_{2 k-2}+x_{2 k-1} \geq 2(k+1) .
$$

Since $h \geq k$, we have $2(k+1)>2(2 k-h)$.

Case 1: $x_{h} \geq 2$.

Since $2(k+1)>2(2 k-h)$, there exists an integer $b \geq 3$ such that $(b-1)(2 k-h)<2(k+1) \leq$ $b(2 k-h)$. Hence $h \leq \frac{(2 b-2) k-2}{b}<2 k-1$. It follows by $(b-1)(2 k-h)<2(k+1)$ and by $(2.4)$ that $x_{h}+x_{h+1}+\cdots+x_{2 k-2}+x_{2 k-1} \geq b$, and so by (2.2), we have $r_{1} \leq r_{2} \leq \cdots \leq r_{b} \leq 2 k-1$. By Lemma 2.2.8, $\left|V_{i}\right| \geq \delta+1$ with $1 \leq i \leq b$. Let $V^{\prime}=V \backslash\left(V_{1} \cup V_{2}\right)$. Then $\left|V^{\prime}\right| \geq\left|V_{3}\right|+\cdots+\left|V_{b}\right| \geq$ $(b-2)(\delta+1)$. Let $e\left(V_{1}, V_{2}\right)=y$. Then $y \geq 1$. The quotient matrix of $G$ with respect to the partition $\left(V_{1}, V_{2}, V^{\prime}\right)$ is

$$
A_{3}=\left[\begin{array}{ccc}
\bar{d}_{1}-\frac{h}{\left|V_{1}\right|} & \frac{y}{\left|V_{1}\right|} & \frac{h-y}{\left|V_{1}\right|} \\
\frac{y}{\left|V_{2}\right|} & \bar{d}_{2}-\frac{h}{\left|V_{2}\right|} & \frac{h-y}{\left|V_{2}\right|} \\
\frac{h-y}{\left|V^{\prime}\right|} & \frac{h-y}{\left|V^{\prime}\right|} & \overline{d^{\prime}}-\frac{2(h-y)}{\left|V^{\prime}\right|}
\end{array}\right],
$$


where $\bar{d}_{i}$ denotes the average degree of $V_{i}$ in $G$ for $i=1,2$ and $\bar{d}^{\prime}$ denotes the average degree of $V^{\prime}$ in $G$.

By $(2.1), \lambda_{2}\left(A_{3}\right)+\lambda_{3}\left(A_{3}\right)=\operatorname{tr}\left(A_{3}\right)-\lambda_{1}\left(A_{3}\right)$. By Theorem 2.2.7, $\lambda_{2}(G) \geq \lambda_{2}\left(A_{3}\right), \lambda_{3}(G) \geq$ $\lambda_{3}\left(A_{3}\right)$ and by Theorem 2.2.6, $\lambda_{1}\left(A_{3}\right) \leq \max \left\{\bar{d}_{1}, \bar{d}_{2}, \bar{d}^{\prime}\right\}$. Thus $\lambda_{2}(G)+\lambda_{3}(G) \geq \operatorname{tr}\left(A_{3}\right)-$ $\max \left\{\bar{d}_{1}, \bar{d}_{2}, \bar{d}^{\prime}\right\}$. As $\lambda_{2}(G) \geq \lambda_{3}(G)$, we have

$$
\begin{aligned}
2 \lambda_{2}(G) & \geq \operatorname{tr}\left(A_{3}\right)-\max \left\{\bar{d}_{1}, \bar{d}_{2}, \bar{d}^{\prime}\right\} \\
& =\bar{d}_{1}+\bar{d}_{2}+\bar{d}^{\prime}-\left(\frac{h}{\left|V_{1}\right|}+\frac{h}{\left|V_{2}\right|}+\frac{2(h-y)}{\left|V^{\prime}\right|}\right)-\max \left\{\bar{d}_{1}, \bar{d}_{2}, \bar{d}^{\prime}\right\} \\
& \geq 2 \delta-\frac{2\left(\frac{b-1}{b-2} h-\frac{y}{b-2}\right)}{\delta+1}, \\
& \geq 2\left(\delta-\frac{\frac{2(b-1)^{2}}{b(b-2)} k-\frac{3 b-2}{b(b-2)}}{\delta+1}\right),
\end{aligned}
$$

contrary to the assumption in Theorem 2.1.6 (iii) that $\lambda_{2}(G)<\delta-\frac{3 k-1}{\delta+1}$. (To see this, let $f(b)=\frac{2(b-1)^{2}}{b(b-2)}$. Then by Calculus, one can verify that $f(b)$ is a decreasing function of $b$ over the interval $[3, \infty)$, and so for any $b \geq 3, f(b) \leq f(3)=\frac{8}{3}<3$.) This proves Case 1 .

Case 2: $x_{h}=1$.

Then (2.4) becomes $(2 k-h-1) x_{h+1}+\cdots+2 x_{2 k-2}+x_{2 k-1} \geq 2(k+1)-(2 k-h)=h+2 \geq k+2$. Let $h^{\prime}$ be the smallest index such that $x_{h^{\prime}}>0$ with $h^{\prime}>h$. Then

$$
\left(2 k-h^{\prime}\right) x_{h^{\prime}}+\cdots+2 x_{2 k-2}+x_{2 k-1} \geq h+2 \geq k+2 .
$$

As $h^{\prime} \geq h \geq k$, we have $h^{\prime}+2>k$ and so $k+2>2 k-h^{\prime}$. Thus there must be an integer $b^{\prime} \geq 2$ such that $\left(b^{\prime}-1\right)\left(2 k-h^{\prime}\right)<k+2 \leq b^{\prime}\left(2 k-h^{\prime}\right)$. Hence $h^{\prime} \leq \frac{\left(2 b^{\prime}-1\right) k-2}{b^{\prime}}<2 k-1$. By $\left(b^{\prime}-1\right)\left(2 k-h^{\prime}\right)<k+2$ and by $(2.5)$, we have $x_{h^{\prime}}+\cdots+x_{2 k-2}+x_{2 k-1} \geq b^{\prime}$, and so by (2.2), $r_{1} \leq r_{2} \leq \cdots \leq r_{b^{\prime}} \leq r_{b^{\prime}+1} \leq 2 k-1$. By Lemma $2.2 .8,\left|V_{i}\right| \geq \delta+1$ for $i=1,2, \cdots, b^{\prime}+1$. Let $V^{\prime}=V \backslash\left(V_{1} \cup V_{2}\right)$. Then $\left|V^{\prime}\right| \geq\left|V_{3}\right|+\cdots+\left|V_{b^{\prime}+1}\right| \geq\left(b^{\prime}-1\right)(\delta+1)$. Let $e\left(V_{1}, V_{2}\right)=y$. Then $y \geq 1$. The quotient matrix of $G$ with respect to the partition $\left(V_{1}, V_{2}, V^{\prime}\right)$ is

$$
A_{3}=\left[\begin{array}{ccc}
\bar{d}_{1}-\frac{h}{\left|V_{1}\right|} & \frac{y}{\left|V_{1}\right|} & \frac{h-y}{\left|V_{1}\right|} \\
\frac{y}{\left|V_{2}\right|} & \bar{d}_{2}-\frac{h^{\prime}}{\left|V_{2}\right|} & \frac{h^{\prime}-y}{\left|V_{2}\right|} \\
\frac{h-y}{\left|V^{\prime}\right|} & \frac{h^{\prime}-y}{\left|V^{\prime}\right|} & \bar{d}^{\prime}-\frac{h+h^{\prime}-2 y}{\left|V^{\prime}\right|}
\end{array}\right],
$$

where $\bar{d}_{i}$ denotes the average degree of $V_{i}$ in $G$ for $i=1,2$ and $\bar{d}^{\prime}$ denotes the average degree of $V^{\prime}$ in $G$.

By $(2.1), \lambda_{2}\left(A_{3}\right)+\lambda_{3}\left(A_{3}\right)=\operatorname{tr}\left(A_{3}\right)-\lambda_{1}\left(A_{3}\right)$. By Theorem 2.2.7, $\lambda_{2}(G) \geq \lambda_{2}\left(A_{3}\right), \lambda_{3}(G) \geq$ $\lambda_{3}\left(A_{3}\right)$ and by Theorem 2.2.6, $\lambda_{1}\left(A_{3}\right) \leq \max \left\{\bar{d}_{1}, \bar{d}_{2}, \bar{d}^{\prime}\right\}$. Thus $\lambda_{2}(G)+\lambda_{3}(G) \geq \operatorname{tr}\left(A_{3}\right)-$ 
$\max \left\{\bar{d}_{1}, \bar{d}_{2}, \bar{d}^{\prime}\right\}$. As $\lambda_{2}(G) \geq \lambda_{3}(G)$, we have

$$
\begin{aligned}
2 \lambda_{2}(G) & \geq \operatorname{tr}\left(A_{3}\right)-\max \left\{\bar{d}_{1}, \bar{d}_{2}, \bar{d}^{\prime}\right\} \\
& =\bar{d}_{1}+\bar{d}_{2}+\bar{d}^{\prime}-\left(\frac{h}{\left|V_{1}\right|}+\frac{h^{\prime}}{\left|V_{2}\right|}+\frac{\left.h+h^{\prime}-2 y\right)}{\left|V^{\prime}\right|}\right)-\max \left\{\bar{d}_{1}, \bar{d}_{2}, \bar{d}^{\prime}\right\} \\
& \geq 2 \delta-\frac{b^{\prime} h+b^{\prime} h^{\prime}-2 y}{\left(b^{\prime}-1\right)(\delta+1)} \geq 2 \delta-\frac{2\left(b^{\prime} h^{\prime}-y\right)}{\left(b^{\prime}-1\right)(\delta+1)}, \\
& \geq 2\left(\delta-\frac{\frac{2 b^{\prime}-1}{b^{\prime}-1} k-\frac{3}{b^{\prime}-1}}{\delta+1}\right),
\end{aligned}
$$

contrary to the assumption in Theorem 2.1.6 (iii) that $\lambda_{2}(G)<\delta-\frac{3 k-1}{\delta+1}$. (To see this, let $g\left(b^{\prime}\right)=\frac{2 b^{\prime}-1}{b^{\prime}-1}$. Then by Calculus, one can verify that $g\left(b^{\prime}\right)$ is a decreasing function of $b^{\prime}$ over the interval $[2, \infty)$, and so for any $b^{\prime} \geq 2, g\left(b^{\prime}\right) \leq g(2)=3$.) This completes the proof.

\subsection{Laplacian and signless Laplacian eigenvalue conditions}

In this section, we will investigate the relationship between $\mu_{n-1}(G), q_{2}(G)$ and $\tau(G), \kappa^{\prime}(G)$ of a simple graph $G$. Theorem 2.5.3 and 2.5.4 are main results, which are analogues of Theorem 2.1.5 and Theorem 2.1.6. We present a useful theorem first.

Theorem 2.5.1. (So [66]) Let $B$ and $C$ be Hermitian matrices of order $n$, and let $1 \leq i, j \leq n$. Then

(i) $\lambda_{i}(B)+\lambda_{j}(C) \leq \lambda_{i+j-n}(B+C)$ if $i+j \geq n+1$.

(ii) $\lambda_{i}(B)+\lambda_{j}(C) \geq \lambda_{i+j-1}(B+C)$ if $i+j \leq n+1$.

Corollary 2.5.2. Let $\delta, \Delta, \lambda_{2}, \mu_{n-1}$ and $q_{2}$ be the minimum degree, maximum degree, second largest eigenvalue, second smallest Laplacian eigenvalue and second largest signless Laplacian eigenvalue of a graph $G$. Then

(i) $\mu_{n-1}+\lambda_{2} \leq \Delta$.

(ii) $\delta+\lambda_{2} \leq q_{2}$.

Proof: Let $A, D, L, Q$ be the adjacency matrix, diagonal matrix, Laplacian matrix and signless Laplacian matrix.

(i): Since $L=D-A$, we have $D=L+A$. By Theorem 2.5.1 (i), $\lambda_{n-1}(L)+\lambda_{2}(A) \leq \lambda_{1}(D)$. Thus $\mu_{n-1}+\lambda_{2} \leq \Delta$.

(ii): Since $Q=D+A$, by Theorem 2.5.1 (i), $\lambda_{n}(D)+\lambda_{2}(A) \leq \lambda_{2}(Q)$. Thus $\delta+\lambda_{2} \leq q_{2}$.

Theorem 2.5.3. Let $k \geq 2$ be an integer, $G$ be a graph with minimum degree $\delta$.

(i) If $\delta \geq 4$ and $\mu_{n-1}(G)>\Delta-\delta+\frac{3}{\delta+1}$, then $\tau(G) \geq 2$.

(ii) If $\delta \geq 6$ and $\mu_{n-1}(G)>\Delta-\delta+\frac{5}{\delta+1}$, then $\tau(G) \geq 3$.

(iii) For $k \geq 4$, if $\delta \geq 2 k$ and $\mu_{n-1}(G)>\Delta-\delta+\frac{3 k-1}{\delta+1}$, then $\tau(G) \geq k$.

(iv) For $k \geq 2$ and $\delta \geq k$, if $\mu_{n-1}(G)>\Delta-\delta+\frac{2(k-1)}{\delta+1}$, then $\kappa^{\prime}(G) \geq k$. 
Proof: By Corollary 2.5.2 and Theorem 2.1.6.

Theorem 2.5.4. Let $k \geq 2$ be an integer, $G$ be a graph with minimum degree $\delta$.

(i) If $\delta \geq 4$ and $q_{2}(G)<2 \delta-\frac{3}{\delta+1}$, then $\tau(G) \geq 2$.

(ii) If $\delta \geq 6$ and $q_{2}(G)<2 \delta-\frac{5}{\delta+1}$, then $\tau(G) \geq 3$.

(iii) For $k \geq 4$, if $\delta \geq 2 k$ and $q_{2}(G)<2 \delta-\frac{3 k-1}{\delta+1}$, then $\tau(G) \geq k$.

(iv) For $k \geq 2$ and $\delta \geq k$, if $q_{2}(G)<2 \delta-\frac{2(k-1)}{\delta+1}$, then $\kappa^{\prime}(G) \geq k$.

Proof: By Corollary 2.5.2 and Theorem 2.1.6. 


\section{Chapter 3}

\section{Strength extremal graphs}

\subsection{Introduction}

With graphs considered as natural models for many network design problems, edge connectivity and maximum number of edge-disjoint spanning trees of a graph have been used as measures for reliability and strength in communication networks modeled as a graph (see [21,54], among others).

We consider finite graphs with possible multiple edges in this chapter. For any graph $G$, we define $\overline{\kappa^{\prime}}(G)=\max \left\{\kappa^{\prime}(H): H\right.$ is a subgraph of $\left.G\right\}$. The invariant $\overline{\kappa^{\prime}}(G)$, first introduced by Matula [53], has been studied by Boesch and McHugh [4], by Lai [43], by Matula [53, 54], by Mitchem [56] and implicitly by Mader [52]. In [54], Matula gave a polynomial algorithm to determine $\overline{\kappa^{\prime}}(G)$.

Throughout this chapter, $k$ and $n$ denote positive integers, unless otherwise defined.

Mader in [52] first introduced $k$-maximal graphs. A graph $G$ is $k$-maximal if $\overline{\kappa^{\prime}}(G) \leq k$ but for any edge $e \notin E(G), \overline{\kappa^{\prime}}(G+e) \geq k+1$. The $k$-maximal graphs have been studied in $[4,43,52-54,56]$, among others.

Simple $k$-maximal graphs have been well studied. In [52], Mader proved that the maximum number of edges in a simple $k$-maximal graph with $n$ vertices is $(n-k) k+\left(\begin{array}{l}k \\ 2\end{array}\right)$ and characterized all the extremal graphs. In 1990, Lai [43] showed that the minimum number of edges in a simple $k$-maximal graph with $n$ vertices is $(n-1) k-\left(\begin{array}{c}k \\ 2\end{array}\right)\left\lfloor\frac{n}{k+2}\right\rfloor$. In the same paper, Lai also characterized all the extremal graphs and all the simple $k$-maximal graphs.

This chapter mainly focus on multiple $k$-maximal graphs, and we show that the number of edges in a $k$-maximal graph with $n$ vertices is $k(n-1)$ and give a complete characterization of all $k$-maximal graphs as well as show several equivalent graph families.

When a network is modeled as a graph $G$, both $\kappa^{\prime}(G)$ and $\tau(G)$ have been used as measures of the strength or reliability of the network (see $[21,54]$ ). As it is known that for any connected 
graph $G, \kappa^{\prime}(G) \geq \tau(G)$, it is natural to ask when the equality holds. Motivated by this question, we characterized all graphs $G$ satisfying $\kappa^{\prime}(G)=\tau(G)$ with minimum number of possible edges for a fixed number of vertices. We also investigate necessary and sufficient conditions for a graph to have a spanning subgraph with this property or to be a spanning subgraph of another graph with this property.

In Section 3.2, we will characterize all $k$-maximal graphs. The characterizations of minimal graphs with $\kappa^{\prime}=\tau$ and reinforcement problems will be discussed in Sections 3.3 and 3.4, respectively.

\subsection{Characterizations of $k$-maximal graphs}

In this section, we shall present a structural characterization of $k$-maximal graphs as well as several equivalent classes of graphs, as shown in Theorem 3.2.1.

Let $F(n, k)$ be the maximum number of edges in a graph $G$ on $n$ vertices that does not contain a subgraph $H$ with $\overline{\kappa^{\prime}}(H) \geq k+1$. We define $\mathcal{F}(n, k)=\{G:|E(G)|=F(n, k),|V(G)|=$ $\left.n, \overline{\kappa^{\prime}}(G) \leq k\right\}$.

Let $G_{1}$ and $G_{2}$ be connected graphs such that $V\left(G_{1}\right) \cap V\left(G_{2}\right)=\emptyset$. Let $K$ be a set of $k$ edges each of which has one vertex in $V\left(G_{1}\right)$ and the other vertex in $V\left(G_{2}\right)$. The $K$-edge-join $G_{1} *_{K} G_{2}$ is defined to be the graph with vertex set $V\left(G_{1}\right) \cup V\left(G_{2}\right)$ and edge set $E\left(G_{1}\right) \cup E\left(G_{2}\right) \cup K$. When the set $K$ is not emphasized, we use $G_{1} *_{k} G_{2}$ for $G_{1} *_{K} G_{2}$, and refer $G_{1} *_{k} G_{2}$ as a $k$-edge-join.

Let $\mathcal{G}_{k}$ be a family of graphs such that for any $G_{1}, G_{2} \in \mathcal{G}_{k} \cup\left\{K_{1}\right\}, G_{1} *_{k} G_{2} \in \mathcal{G}_{k}$. Let $\bar{\tau}(G)=\max \{\tau(H): H$ is a subgraph of $G\}$. The main theorem in this section is stated below.

Theorem 3.2.1. Let $G$ be a graph on $n$ vertices. The following statements are equivalent.

(i) $G \in \mathcal{F}(n, k)$;

(ii) $G$ is k-maximal;

(iii) $\eta(G)=\overline{\kappa^{\prime}}(G)=k$;

(iv) $\tau(G)=\overline{\kappa^{\prime}}(G)=k$;

(v) $\tau(G)=\bar{\tau}(G)=\kappa^{\prime}(G)=\overline{\kappa^{\prime}}(G)=k$;

(vi) $G \in \mathcal{G}_{k}$.

In order to prove Theorem 3.2.1, we need some lemmas.

For a connected graph $G$ with $\tau(G) \geq k$, we define $E_{k}(G)=\{e \in E(G): \tau(G-e) \geq k\}$.

Lemma 3.2.2. (Lai et al. [47], Li [46])

Let $G$ be a connected graph with $\tau(G) \geq k$. Then $E_{k}(G)=\emptyset$ if and only if $d(G)=k$.

Lemma 3.2.3. (Haas [37], Liu et al. [48], and Lai et al. [45])

The following statements are equivalent for a graph $G$. 
(i) $\gamma(G) \leq k$.

(ii) There exist $k(|V(G)|-1)-|E(G)|$ edges which when added to $G$ result in a graph that can be decomposed into $k$ edge-disjoint spanning trees.

Lemma 3.2.4. Let $X$ be a $k$-edge cut of a graph $G$. If $H$ is a subgraph of $G$ with $\kappa^{\prime}(H)>k$, then $E(H) \cap X=\emptyset$.

Proof: If $E(H) \cap X \neq \emptyset$, then $\kappa^{\prime}(H) \leq|E(H) \cap X| \leq|X|=k<\kappa^{\prime}(H)$, a contradiction.

Lemma 3.2.5. If a graph $G$ is $k$-maximal, then $\kappa^{\prime}(G)=\overline{\kappa^{\prime}}(G)=k$.

Proof: Since $G$ is $k$-maximal, $\kappa^{\prime}(G) \leq \overline{\kappa^{\prime}}(G) \leq k$. It suffices to show that $\kappa^{\prime}(G)=k$. We assume that $\kappa^{\prime}(G)<k$ and prove it by contradiction. Let $X$ be an edge cut with $|X|<k$ and suppose that $G=G_{1} *_{X} G_{2}$. Let $e \notin E(G)$ be an edge with one end in $V\left(G_{1}\right)$ and the other end in $V\left(G_{2}\right)$. By the definition of $k$-maximal graphs, $\overline{\kappa^{\prime}}(G+e) \geq k+1$. Thus $G+e$ has a subgraph $H$ with $\kappa^{\prime}(H) \geq k+1$. Then it must be the case that $e \in E(H)$, otherwise $H$ is a subgraph of $G$, contrary to $\overline{\kappa^{\prime}}(G) \leq k$. Since $X \cup\{e\}$ is an edge cut of $G+e$ with $|X \cup\{e\}| \leq k$ and $H$ is a subgraph of $G+e$ with $\kappa^{\prime}(H) \geq k+1$, by Lemma 3.2.4, $E(H) \cap(X \cup\{e\})=\emptyset$, contrary to $e \in E(H)$.

Lemma 3.2.6. If a graph $G$ is k-maximal, then $G=G_{1} *_{k} G_{2}$ where either $G_{i}=K_{1}$ or $G_{i}$ is $k$-maximal for $i=1,2$.

Proof: By Lemma 3.2.5, $G$ has a $k$-edge cut $X$, and so $G=G_{1} *_{k} G_{2}$. For $i=1,2$, suppose that $G_{i} \neq K_{1}$, we want to prove that $G_{i}$ is $k$-maximal. Since $G$ is $k$-maximal, $\overline{\kappa^{\prime}}(G) \leq k$, whence $\kappa^{\prime}\left(G_{i}\right) \leq k$. For any edge $e \notin E\left(G_{i}\right), \overline{\kappa^{\prime}}(G+e) \geq k+1$. Thus $G+e$ has a subgraph $H$ with $\kappa^{\prime}(H) \geq k+1$. Since $\overline{\kappa^{\prime}}(G) \leq k, H$ is not a subgraph of $G$, and so $e \in E(H)$. Since $X$ is a $k$-edge cut of $G+e$, by Lemma 3.2.4, $E(H) \cap X=\emptyset$. Hence $H$ is a subgraph of $G_{i}+e$ with $\kappa^{\prime}(H) \geq k+1$, whence $\overline{\kappa^{\prime}}\left(G_{i}\right) \geq k+1$. Thus $G_{i}$ is $k$-maximal.

Lemma 3.2.7. Let $G$ be a graph on $n$ vertices. Then $G \in \mathcal{F}(n, k)$ if and only if $G$ is $k$-maximal.

Proof: By the definition of $\mathcal{F}(n, k)$, if $G \in \mathcal{F}(n, k)$, then $|E(G)|=F(n, k)$ and $\overline{\kappa^{\prime}}(G) \leq k$. Then for any edge $e \notin E(G),|E(G+e)|=|E(G)|+1>F(n, k)$, and so $\overline{\kappa^{\prime}}(G+e) \geq k+1$. By the definition of $k$-maximal graphs, $G$ is $k$-maximal.

Now we assume that $G$ is $k$-maximal to prove that $G \in \mathcal{F}(n, k)$. It suffices to show that $|E(G)|=F(n, k)=k(n-1)$ by induction on $n$. When $n=2, G$ is $k K_{2}$, which is the graph with 2 vertices and $k$ multiple edges, and so $|E(G)|=k$. We assume that $|E(G)|=F(n, k)=k(n-1)$ holds for smaller values of $n>2$. By Lemma 3.2.6, $G=G_{1} *_{k} G_{2}$ where $G_{i}$ is $k$-maximal or $k_{1}$ for $i=1,2$. Let $\left|V\left(G_{i}\right)\right|=n_{i}$. By inductive hypothesis, $\left|E\left(G_{i}\right)\right|=k\left(n_{i}-1\right)$. Thus $|E(G)|=k\left(n_{1}-1\right)+k\left(n_{2}-1\right)+k=k(n-1)$. 
Lemma 3.2.8. $F(n, k)=k(n-1)$.

Proof: By Lemma 3.2.7, it suffices to show that for any $k$-maximal graph $G$ with $|V(G)|=n$, $|E(G)|=k(n-1)$. We argue by induction on $n$. When $n=2$, by Lemma 3.2.6, $G$ is a graph with $n=2$ vertices and $k$ multiple edges, and thus $|E(G)|=k(n-1)$. Assume that the statement holds for smaller value of $n>2$. By Lemma 3.2.6, $G=G_{1} *_{k} G_{2}$ where either $G_{i}=K_{1}$ or $G_{i}$ is $k$-maximal, for $i=1,2$. By inductive hypothesis, $\left|E\left(G_{i}\right)\right|=k\left(\left|V\left(G_{i}\right)\right|-1\right)$. Then $|E(G)|=\left|E\left(G_{1}\right)\right|+\left|E\left(G_{2}\right)\right|+k=k\left(\left|V\left(G_{1}\right)\right|+\left|V\left(G_{2}\right)\right|-2\right)+k=k(n-1)$, completing the proof.

Lemma 3.2.9. Suppose $\tau(G)=\bar{\tau}(G)=\kappa^{\prime}(G)=\overline{\kappa^{\prime}}(G)=k$. Then $G=G_{1} *_{k} G_{2}$ where either $G_{i}=K_{1}$ or $G_{i}$ satisfies $\tau\left(G_{i}\right)=\bar{\tau}\left(G_{i}\right)=\kappa^{\prime}\left(G_{i}\right)=\overline{\kappa^{\prime}}\left(G_{i}\right)=k$ for $i=1,2$.

Proof: Since $\kappa^{\prime}(G)=k$, there must be an edge-cut of size $k$. Hence there exist graphs $G_{1}$ and $G_{2}$ such that $G=G_{1} *_{k} G_{2}$. If $G_{i} \neq K_{1}$, we will prove $\tau\left(G_{i}\right)=\bar{\tau}\left(G_{i}\right)=\kappa^{\prime}\left(G_{i}\right)=\overline{\kappa^{\prime}}\left(G_{i}\right)=k$, for $i=1,2$. First, by the definition of $\bar{\tau}, \tau\left(G_{i}\right) \leq \bar{\tau}\left(G_{i}\right) \leq \bar{\tau}(G)=k$ for $i=1,2$. Since $G$ has $k$ disjoint spanning trees, we have $\tau\left(G_{i}\right) \geq k$ for $i=1,2$. Thus $\tau\left(G_{i}\right)=\bar{\tau}\left(G_{i}\right)=k$ for $i=1,2$. Now we prove $\kappa^{\prime}\left(G_{i}\right)=\overline{\kappa^{\prime}}\left(G_{i}\right)=k$ for $i=1,2$. Since $\overline{\kappa^{\prime}}(G)=k, \kappa^{\prime}\left(G_{i}\right) \leq \overline{\kappa^{\prime}}\left(G_{i}\right) \leq k$. But $\kappa^{\prime}\left(G_{i}\right) \geq \tau\left(G_{i}\right)=k$ for $i=1,2$. Hence we have $\tau\left(G_{i}\right)=\bar{\tau}\left(G_{i}\right)=\kappa^{\prime}\left(G_{i}\right)=\overline{\kappa^{\prime}}\left(G_{i}\right)=k$ for $i=1,2$.

Lemma 3.2.10. Let $G=G_{1} *_{k} G_{2}$ where $G_{i}=K_{1}$ or $G_{i}$ satisfies $\tau\left(G_{i}\right)=\bar{\tau}\left(G_{i}\right)=\kappa^{\prime}\left(G_{i}\right)=$ $\overline{\kappa^{\prime}}\left(G_{i}\right)=k$ for $i=1,2$. Then $\tau(G)=\bar{\tau}(G)=\kappa^{\prime}(G)=\overline{\kappa^{\prime}}(G)=k$.

Proof: Since $G=G_{1} *_{k} G_{2}$ and $\kappa^{\prime}\left(G_{1}\right)=\kappa^{\prime}\left(G_{2}\right)=k$, we have $\tau(G) \leq \kappa^{\prime}(G)=k$ and there exists an edge-cut $X=\left\{x_{1}, x_{2}, \cdots, x_{k}\right\}$ such that $G=G_{1} *_{X} G_{2}$. Let $T_{1, i}, T_{2, i}, \cdots, T_{k, i}$ be edgedisjoint spanning trees of $G_{i}$, for $i=1,2$. Then $T_{1,1}+x_{1}+T_{1,2}, T_{2,1}+x_{2}+T_{2,2}, \cdots, T_{k, 1}+x_{k}+T_{k, 2}$ are $k$ edge-disjoint spanning trees of $G$. Thus $\tau(G)=\kappa^{\prime}(G)=k$. Now we need to prove that for any subgraph $H$ of $G, \tau(H) \leq k$ and $\kappa^{\prime}(H) \leq k$. If $E(H) \cap X \neq \emptyset$, then $E(H) \cap X$ is an edge cut of $H$ and thus $\tau(H) \leq \kappa^{\prime}(H) \leq k$. If $E(H) \cap X=\emptyset$, then $H$ is a spanning subgraph of either $G_{1}$ or $G_{2}$, whence $\tau(H) \leq \kappa^{\prime}(H) \leq k$.

Now we present the proof of Theorem 3.2.1.

Proof of Theorem 3.2.1: By Lemma 3.2.7, (i) and (ii) are equivalent. By (1.3), (iii) $\Rightarrow$ (iv). (i) $\Rightarrow$ (iii): By Lemma 3.2.8, $|E(G)|=k(n-1)$. By the definition of $d(G), d(G)=k$. Since $\overline{\kappa^{\prime}}(G) \leq k$, for any subgraph $H$ of $G, \overline{\kappa^{\prime}}(H) \leq k$. Hence $|E(H)| \leq k(|V(H)|-1)$, whence $d(H) \leq k$. By the definition of $\gamma(G)$, we have $\gamma(G) \leq k$. Thus $d(G)=\gamma(G)=k$. By Theorem 1.1.3, $\eta(G)=k$. Hence $k=\eta(G)=\tau(G) \leq \overline{\kappa^{\prime}}(G) \leq k$, i.e., $\eta(G)=\overline{\kappa^{\prime}}(G)=k$. (iv) $\Rightarrow$ (i): By Lemma 3.2.8, $|E(G)| \leq k(n-1)$. Since $\tau(G)=k, G$ has $k$ edge-disjoint spanning 
trees, and so $|E(G)| \geq k(n-1)$. Thus $|E(G)|=k(n-1)$, and so $G \in \mathcal{F}(n, k)$.

(iv) $\Leftrightarrow(\mathrm{v})$ : By definition, $\tau(G) \leq \bar{\tau}(G) \leq \overline{\kappa^{\prime}}(G)$ and $\tau(G) \leq \kappa^{\prime}(G) \leq \overline{\kappa^{\prime}}(G)$. The equivalence between (iv) and (v) now follow from these inequalities.

(v) $\Rightarrow($ vi): We argue by induction on $|V(G)|$. When $|V(G)|=2$, a graph $G$ with $\tau(G)=\bar{\tau}(G)=$ $\kappa^{\prime}(G)=\overline{\kappa^{\prime}}(G)=k$ must be $K_{1} *_{k} K_{1}$, and so by definition, $G \in \mathcal{G}_{k}$. We assume that (v) $\Rightarrow$ (vi) holds for smaller values of $|V(G)|$. By Lemma 3.2.9, $G=G_{1} *_{k} G_{2}$ with $\tau\left(G_{i}\right)=\bar{\tau}\left(G_{i}\right)=$ $\kappa^{\prime}\left(G_{i}\right)=\overline{\kappa^{\prime}}\left(G_{i}\right)=k$ or $G_{i}=K_{1}$, for $i=1,2$. If $G_{i} \neq K_{1}$, then by the inductive hypothesis, $G_{i} \in \mathcal{G}_{k}$. By definition, $G \in \mathcal{G}_{k}$.

(vi) $\Rightarrow(\mathrm{v})$ : We show it by induction on $|V(G)|$. When $|V(G)|=2$, by the definition of $\mathcal{G}_{k}$, $G=K_{1} *_{k} K_{1}$, and then $\tau(G)=\bar{\tau}(G)=\kappa^{\prime}(G)=\overline{\kappa^{\prime}}(G)=k$. We assume that it holds for smaller values of $|V(G)|$. By the definition of $\mathcal{G}_{k}, G=G_{1} *_{k} K_{1}$ or $G=G_{1} *_{k} G_{2}$ where $G_{1}, G_{2} \in \mathcal{G}_{k}$. By inductive hypothesis, $\tau\left(G_{i}\right)=\bar{\tau}\left(G_{i}\right)=\kappa^{\prime}\left(G_{i}\right)=\overline{\kappa^{\prime}}\left(G_{i}\right)=k$ for $i=1,2$, and by Lemma 3.2.10, $\tau(G)=\bar{\tau}(G)=\kappa^{\prime}(G)=\overline{\kappa^{\prime}}(G)=k$.

\subsection{Characterizations of minimal graphs with $\kappa^{\prime}=\tau$}

We define

$$
\mathcal{F}_{k, n}=\left\{G: \kappa^{\prime}(G)=\tau(G)=k,|V(G)|=n \text { and }|E(G)| \text { is minimized }\right\}
$$

and $\mathcal{F}_{k}=\cup_{n>1} \mathcal{F}_{k, n}$.

In this section, we will give characterizations of graphs in $\mathcal{F}_{k}$. In addition, we use $\mathcal{F}_{k, n}$ to characterize graphs $G$ with $\kappa^{\prime}(G)=\tau(G)$.

Theorem 3.3.1. Let $G$ be a graph, then $G \in \mathcal{F}_{k}$ if and only if $G$ satisfies

(i) $G$ has an edge-cut of size $k$, and

(ii) $G$ is uniformly dense with density $k$.

Proof: Suppose that $G \in \mathcal{F}_{k}$, then $\tau(G)=\kappa^{\prime}(G)=k$. Hence $G$ has an edge-cut of size $k$. Since $|E(G)|$ is minimized, we have $E_{k}(G)=\emptyset$, and by Lemma 3.2.2, $d(G)=k$. Since $\tau(G)=k$, by Theorem 1.1.1 and the definition of $\eta(G)$, we have $\eta(G) \geq k$. By $(1.2), \eta(G) \leq d(G)=k$, whence $\eta(G)=d(G)=k$, and thus $G$ is uniformly dense with density $k$.

On the other hand, suppose that $G$ satisfies (i) and (ii). By (ii) and Theorem 1.1.3, $\eta(G)=$ $d(G)=k$. By (1.3), $\tau(G)=k$. Then $\kappa^{\prime}(G) \geq \tau(G)=k$. But $G$ has an edge-cut of size $k$, thus $\kappa^{\prime}(G)=\tau(G)=k$. Since $d(G)=k$, by Lemma 3.2.2, $E_{k}(G)=\emptyset$, i.e. $|E(G)|$ is minimized. Thus $G \in \mathcal{F}_{k}$.

Theorem 3.3.2. A graph $G \in \mathcal{F}_{k}$ if and only if $G=G_{1} *_{k} G_{2}$ where either $G_{i}=K_{1}$ or $G_{i}$ is uniformly dense with density $k$ for $i=1,2$. 
Proof: Suppose that $G \in \mathcal{F}_{k}$. By Theorem 3.3.1, $G$ has an edge-cut of size $k$, whence there exist graphs $G_{1}$ and $G_{2}$ such that $G=G_{1} *_{k} G_{2}$. Now we will prove that $G_{i}$ is uniformly dense with density $k$ if it is not isomorphic to $K_{1}$, for $i=1,2$. Since $\tau(G)=k$, we have $\tau\left(G_{i}\right) \geq k$, and thus $d\left(G_{i}\right) \geq k$, for $i=1,2$. By (1.2), (1.3) and Theorem 1.1.3, it suffices to prove that $d\left(G_{i}\right)=k$ for $i=1,2$. If not, then either $d\left(G_{1}\right)>k$ or $d\left(G_{2}\right)>k$. By (1.1), $|E(G)|=\left|E\left(G_{1}\right)\right|+\left|E\left(G_{2}\right)\right|+k>k\left(\left|V\left(G_{1}\right)\right|-1\right)+k\left(\left|V\left(G_{2}\right)\right|-1\right)+k=k(|V(G)|-1)$, and thus $d(G)=\frac{|E(G)|}{|V(G)|-1}>k$, contrary to the fact that $d(G)=k$. Hence $d\left(G_{i}\right)=k$, and $k \leq \tau\left(G_{i}\right) \leq \eta\left(G_{i}\right) \leq d\left(G_{i}\right)=k$. By Theorem 1.1.3, $G_{i}$ is uniformly dense with density $k$ for $i=1,2$. This proves the necessity.

To prove the sufficiency, first notice that $G$ must have an edge-cut of size $k$, by the definition of the $k$-edge-join. In order to prove $G \in \mathcal{F}_{k}$, by Theorem 3.3.1, it suffices to show that $G$ is uniformly dense with density $k$. Without loss of generality, we may assume that $G_{i}$ is not isomorphic to $K_{1}$ for $i=1,2$. Then $\eta\left(G_{i}\right)=d\left(G_{i}\right)=k$ for $i=1,2$. By $(1.3), \tau\left(G_{i}\right)=\left\lfloor\eta\left(G_{i}\right)\right\rfloor=$ $k$. Also we have $d\left(G_{i}\right)=\frac{\left|E\left(G_{i}\right)\right|}{\left|V\left(G_{i}\right)\right|-1}=k$ for $i=1,2$. Hence $E(G)=\left|E\left(G_{1}\right)\right|+\left|E\left(G_{2}\right)\right|+k=$ $k\left(\left|V\left(G_{1}\right)\right|-1\right)+k\left(\left|V\left(G_{2}\right)\right|-1\right)+k=k(|V(G)|-1)$, whence $d(G)=\frac{|E(G)|}{|V(G)|-1}=k$. Thus $k=\tau(G) \leq \eta(G) \leq d(G)=k$, i.e., $\eta(G)=d(G)=k$, and by Theorem 1.1.3, $G$ is uniformly dense with density $k$. By Theorem 3.3.1, $G \in \mathcal{F}_{k}$.

Theorem 3.3.2 has the following corollary, presenting a recursive structural characterization of graphs in $\mathcal{F}_{k}$.

Corollary 3.3.3. Let $\mathcal{K}(k)=\left\{G: \kappa^{\prime}(G)>\eta(G)=d(G)=k\right\}$. Then a graph $G \in \mathcal{F}_{k}$ if and only if $G=\left(\left(G_{1} *_{k} G_{2}\right) *_{k} \cdots\right) *_{k} G_{t}$ for some integer $t \geq 2$ and $G_{i} \in \mathcal{K}(k) \cup\left\{K_{1}\right\}$ for $i=1,2, \cdots, t$.

Now we can characterize all the graphs $G$ with $\kappa^{\prime}(G)=\tau(G)=k$.

Theorem 3.3.4. A graph $G$ with $n$ vertices satisfies $\kappa^{\prime}(G)=\tau(G)=k$ if and only if $G$ has an edge-cut of size $k$ and a spanning subgraph in $\mathcal{F}_{k, n}$.

Proof: First, suppose that $G$ satisfies $\kappa^{\prime}(G)=\tau(G)=k$. Then $G$ must have an edge-cut $C$ of size $k$ since $\kappa^{\prime}(G)=k$. Hence, $G=G_{1} *_{C} G_{2}$ where $\tau\left(G_{i}\right) \geq k$ or $G_{i}=K_{1}$ for $i=1,2$. If $G_{i}=K_{1}$, then let $G_{i}^{\prime}=K_{1}$. Otherwise, $G_{i}$ must have $k$ edge-disjoint spanning trees $T_{1}, T_{2}, \cdots, T_{k}$, and let $G_{i}^{\prime}$ be the graph with $V\left(G_{i}^{\prime}\right)=V\left(G_{i}\right)$ and $E\left(G_{i}^{\prime}\right)=\cup_{j=1}^{k} E\left(T_{j}\right)$. Let $G^{\prime}=G_{1}^{\prime} *_{C} G_{2}^{\prime}$. Then $G^{\prime}$ is a spanning subgraph of $G$ with $\kappa^{\prime}\left(G^{\prime}\right)=k$ and $k=\tau\left(G^{\prime}\right) \leq \eta\left(G^{\prime}\right) \leq d\left(G^{\prime}\right)=k$. By Theorem 3.3.1, $G^{\prime} \in \mathcal{F}_{k}$. Since $\left|V\left(G^{\prime}\right)\right|=n, G^{\prime} \in \mathcal{F}_{k, n}$, completing the proof of necessity.

To prove the sufficiency, first notice that $\kappa^{\prime}(G) \leq k$, since $G$ has an edge-cut of size $k$. Graph $G$ has a spanning subgraph $G^{\prime} \in \mathcal{F}_{k, n}$, so $\tau\left(G^{\prime}\right)=k$, whence $\tau(G) \geq k$. Thus $k \leq \tau(G) \leq$ $\kappa^{\prime}(G) \leq k$, and we have $\kappa^{\prime}(G)=\tau(G)=k$. 


\subsection{Extensions and restrictions with respect to $\mathcal{F}_{k, n}$}

Let $G$ be a connected graph with $n$ vertices and $H \in \mathcal{F}_{k, n}$. If $G$ is a spanning subgraph of $H$, then $H$ is an $\mathcal{F}_{k, n}$-extension of $G$. If $H$ is a spanning subgraph of $G$, then $H$ is an $\mathcal{F}_{k, n}$-restriction of $G$.

Theorem 3.4.1. Let $G$ be a connected graph with $n$ vertices. Then each of the following holds. (i) $G$ has an $\mathcal{F}_{k, n}$-restriction if and only if $G=G_{1} *_{k^{\prime}} G_{2}$ for some $k^{\prime} \geq k$ and graph $G_{i}$ with $\eta\left(G_{i}\right) \geq k$ or $G_{i}=K_{1}$, for $i=1,2$.

(ii) $G$ has an $\mathcal{F}_{k, n}$-extension if and only if $\kappa^{\prime}(G) \leq k$ and $\gamma(G) \leq k$.

Proof: (i) Suppose that $G$ has an $\mathcal{F}_{k, n}$-restriction $H$, by Theorem 3.3.2, $H=H_{1} *_{k} H_{2}$ where $\tau\left(H_{i}\right)=\eta\left(H_{i}\right)=d\left(H_{i}\right)=k$ or $H_{i}=K_{1}$ for $i=1,2$. Since $H$ is a spanning subgraph of $G$, we have $G=G_{1} *_{k^{\prime}} G_{2}$ for some $k^{\prime} \geq k$ such that $H_{i}$ is a spanning subgraph of $G_{i}$ for $i=1,2$. If $H_{i}=K_{1}$, then $G_{i}=K_{1}$, otherwise, $\eta\left(G_{i}\right) \geq \tau\left(G_{i}\right) \geq \tau\left(H_{i}\right)=k$ for $i=1,2$, by Formula (1.3).

To prove the sufficiency, it suffices to show that $G$ has a spanning subgraph $H \in \mathcal{F}_{k, n}$. Since $G=G_{1} *_{k^{\prime}} G_{2}$, there exists an edge-cut $X$ of size $k^{\prime}$ such that $G=G_{1} *_{X} G_{2}$. Let $Y$ be a subset of size $k$ of $X$. For $i=1,2$, if $G_{i}=K_{1}$, then let $H_{i}=K_{1}$. Otherwise, $\eta\left(G_{i}\right) \geq k$, and by Formula (1.3), $\tau\left(G_{i}\right)=\left\lfloor\eta\left(G_{i}\right)\right\rfloor \geq k$, and then $G_{i}$ has $k$ edge-disjoint spanning trees $T_{1, i}, T_{2, i}, \cdots, T_{k, i}$. Let $H_{i}$ be the graph with $V\left(H_{i}\right)=V\left(G_{i}\right)$ and $E\left(H_{i}\right)=\cup_{j=1}^{k} E\left(T_{j, i}\right)$, for $i=1,2$. Let $H=H_{1} *_{Y} H_{2}$. Then $H$ is a spanning subgraph of $G$ and $\kappa^{\prime}(H)=\tau(H)=k$. Since $d(H)=k$, by Lemma 3.2.2, $H$ has the minimum number of edges with $\tau(H)=k$. Thus $H \in \mathcal{F}_{k, n}$.

(ii) If $G$ has an $\mathcal{F}_{k, n}$-extension $H$, then $G$ is a spanning subgraph of $H$ and $\kappa^{\prime}(H)=\tau(H)=k$ with minimum number of edges. Then $\kappa^{\prime}(G) \leq k$. By Theorem 3.3.1, $d(H)=k$, i.e. $|E(H)|=$ $k(|V(H)|-1)=k(|V(G)|-1)$. Thus $|E(H)|-|E(G)|=k(|V(G)|-1)-|V(G)|$, and by Lemma $3.2 .3, \gamma(G) \leq k$.

To prove the sufficiency, it suffices to show that there is a graph $H \in \mathcal{F}_{k, n}$ with a spanning subgraph $G$. Let $\kappa^{\prime}(G)=k^{\prime}$, then $k^{\prime} \leq k$, and $G$ has an edge-cut $X$ of size $k^{\prime}$. Hence, $G=$ $G_{1} *_{X} G_{2}$. For $i=1,2$, if $G_{i}=K_{1}$, then let $H_{i}=K_{1}$. Otherwise, since $\gamma(G) \leq k$, by the definition of $\gamma(G)$, we have $\gamma\left(G_{i}\right) \leq k$. By Lemma 3.2.3, $G_{i}$ can be reinforcing to a graph $H_{i}$ which can be decomposed into $k$ edge-disjoint spanning trees. Then $\left|E\left(H_{i}\right)\right|=k\left(\left|V\left(H_{i}\right)\right|-1\right)=k\left(\left|V\left(G_{i}\right)\right|-1\right)$, whence $d\left(H_{i}\right)=k$. Since $k=\tau\left(H_{i}\right) \leq \eta\left(H_{i}\right) \leq d\left(H_{i}\right)=k$, we have $\eta\left(H_{i}\right)=d\left(H_{i}\right)=k$, and by Theorem 1.1.3, $H_{i}$ is uniformly dense, for $i=1,2$. Let $H=H_{1} *_{Y} H_{2}$ where $Y$ is an edge subset of size $k$ with $X \subseteq Y$. Then $G$ is a spanning subgraph of $H$. By Theorem 3.3.2, $H \in \mathcal{F}_{k, n}$, completing the proof of the theorem. 


\section{Chapter 4}

\section{Minimally $(2, l)$-connected graphs}

\subsection{Introduction}

In this chapter, we consider finite graphs.

The connectivity $\kappa(G)$ of a graph $G$ is the minimum number of vertices whose removal produces a disconnected graph or the trivial graph. For an integer $l \geq 2$, Chartrand et al. in [13] defined the $l$-connectivity $\kappa_{l}(G)$ of a graph $G$ to be the minimum number of vertices of $G$ whose removal produces a disconnected graph with at least $l$ components or a graph with fewer than $l$ vertices. Thus $\kappa_{l}(G)=0$ if and only if $\omega(G) \geq l$ or $|V(G)| \leq l-1$. Note that $\kappa_{2}(G)=\kappa(G)$.

For an integer $l \geq 2, l$-edge-connectivity can be similarly defined. In [3], Boesch and Chen defined the $l$-edge-connectivity $\lambda_{l}(G)$ of a connected graph $G$ to be the minimum number of edges whose removal leaves a graph with at least $l$ components if $|V(G)| \geq l$, and $\lambda_{l}(G)=|E(G)|$ if $|V(G)|<l$. Note that $\lambda_{2}(G)=\lambda(G)$.

The generalized connectivity and edge-connectivity have been studied by many. See $[3,13$, $32,33,39,40,59-61,73]$, among others. Let $k \geq 1$, a graph $G$ is called $(k, l)$-connected if $\kappa_{l} \geq k$. A graph $G$ is called minimally $(k, l)$-connected if $\kappa_{l}(G) \geq k$ but $\forall e \in E(G), \kappa_{l}(G-e) \leq k-1$. Let $G$ be a $(k, l)$-connected graph, and $e \in E(G)$. An edge $e \in E(G)$ is essential if $G-e$ is not $(k, l)$-connected. A graph $G$ is called $(k, l)$-edge-connected if $\lambda_{l}(G) \geq k$. A graph $G$ is minimally $(k, l)$-edge-connected if $\lambda_{l}(G) \geq k$ but for any edge $e \in E(G), \lambda_{l}(G-e) \leq k-1$. Therefore, a $(2,2)$-connected graph is just a 2-connected graph, and a (2,2)-edge-connected graph is a 2-edge-connected graph.

Let $\mathscr{F}(n, k, l)$ be the set of all connected and minimally $(k, l)$-connected graphs with $n$ vertices. We define $F(n, k, l)=\max \{|E(G)|: G \in \mathscr{F}(n, k, l)\}$ and $f(n, k, l)=\min \{|E(G)|$ : $G \in \mathscr{F}(n, k, l)\}$. Let $\mathscr{I}(n, k, l)=\{i \in \mathbb{N}: f(n, k, l) \leq i \leq F(n, k, l)$ and $\exists G \in \mathscr{F}(n, k, l)$ such that $|E(G)|=i\}$, which is referred as the $(n, k, l)$-spectrum of $\mathscr{F}(n, k, l)$. We further 
define $\operatorname{Ex}(n, k, l)=\{G: G \in \mathscr{F}(n, k, l),|E(G)|=F(n, k, l)\}$ and $\operatorname{Sat}(n, k, l)=\{G: G \in$ $\mathscr{F}(n, k, l),|E(G)|=f(n, k, l)\}$.

Chaty and Chein presented a structural characterization of minimally $(2,2)$-edge-connected graphs [14]. Hennayake et al. [39] then generalized it to minimally $(k, k)$-edge-connected graphs by presenting a structural characterization of all minimally $(k, k)$-edge-connected graphs. A structural characterization of minimally $(2,2)$-connected graphs was obtained independently by Dirac [23] and by Plummer [64]. A purpose of this paper is to give a characterization of minimally $(2, l)$-connected graphs when $l>2$ (Theorem 4.3.2 and Theorem 4.3.5) by presenting the structures of such graphs.

The value of $F(n, 2,2)$ was discovered independently by Dirac [23] and by Plummer [64] (Theorem 4.2.1 in this paper). Another purpose of this paper is to determine $F(n, 2, l)$ and $f(n, 2, l)$ when $l>2$. The families $\operatorname{Ex}(n, 2, l), \operatorname{Sat}(n, 2, l)$ and $\mathscr{I}(n, 2, l)$ will also be determined in the paper. These extend former results by Dirac [23] and Plummer [64] on minimally $(2,2)$ connected graphs.

In Section 2, we will present some preliminaries as preparations for the proofs. Sections 3 and 4 are devoted to the investigations of the structural characterization of minimally $(2, l)$ connected graphs, and of $F(n, 2, l), f(n, 2, l), \operatorname{Ex}(n, 2, l), \operatorname{Sat}(n, 2, l)$ and $\mathscr{I}(n, 2, l)$, respectively.

\subsection{Preliminaries}

We start with a theorem by Dirac and Plummer. These results were obtained by Dirac and by Plummer independently. A chord of a cycle $C$ in a graph $G$ is an edge in $E(G) \backslash E(C)$ both of whose ends lie on $C$.

Theorem 4.2.1. (Dirac [23] and Plummer [64], see also [5])

(i) A 2-connected graph is minimally 2-connected if and only if no cycle has a chord.

(ii) A minimally 2-connected graph of order $n \geq 4$ has the size at most $2 n-4$. Furthermore, $F(n, 2,2)=2 n-4$ and $\operatorname{Ex}(n, 2,2)=\left\{K_{2, n-2}\right\}$ for $n \geq 4$.

A divalent path $P$ in a graph $G$ is a path all of whose internal vertices have degree 2 in $G$. A lane of a graph $G$ is a maximal divalent path in $G$. For convenience, a cycle is considered as a lane of itself. Let $L$ be a lane in graph $G$, we define $L_{0}$ to be the set of all internal vertices of $L$ if $L$ is not an edge of $G$. If $L$ is an edge $e$ of $G$, then $L_{0}=\{e\}$.

By definition, every edge of a graph $G$ is in a divalent path of $G$. Hence, we have the following observation:

Observation 1. Every edge of a graph $G$ lies in a lane in $G$.

A graph is acyclic if it does not contain a cycle. Otherwise, the graph is called cyclic. A cyclic block of a graph is a block which is not isomorphic to $K_{2}$. Let $G$ be a connected 
graph with blocks $B_{1}, B_{2}, \ldots, B_{s}$ and cut vertices $c_{1}, c_{2}, \ldots, c_{t}$, where $s \geq 1$ and $t \geq 0$. The block-cutvertex graph of $G$, denoted by $b c(G)$, is the graph with vertex set $\left\{B_{1}, B_{2}, \ldots, B_{s}\right\} \cup$ $\left\{c_{1}, c_{2}, \ldots, c_{t}\right\}$ and edge set $\left\{B_{i} c_{j}: c_{j} \in V\left(B_{i}\right)\right\}$ for $1 \leq i \leq s$ and $0 \leq j \leq t$. By definition, the block-cutvertex graph of graph $G$ is a tree, and so it is also called the block tree of $G$.

The distance $d_{G}(x, y)$ of two vertices $x$ and $y$ in a graph $G$ is the length of a shortest $(x, y)$ path in $G$, and if no such path exists, then the distance is set to be $\infty$. Let $G$ be a graph and $U \subseteq V(G)$. The diameter of $U$ in $G$, denoted by $\operatorname{diam}_{G}(U)$, is the greatest distance $d_{G}(x, y)$ for $\forall x, y \in U$. If $U=V(G)$, then the diameter of $G$ is simply denoted as $\operatorname{diam}(G)$.

The local connectivity $\kappa_{G}(x, y)$ of two non-adjacent vertices $x$ and $y$ in a graph $G$ is the minimum number of vertices separating $x$ from $y$. If $x$ and $y$ are adjacent vertices, their local connectivity is defined as $\kappa_{H}(x, y)+1$, where $H=G-x y$.

\subsection{Minimally $(2, l)$-connected graphs}

In this section, we shall present a characterization of minimally $(2, l)$-connected graphs.

Lemma 4.3.1. Let $G$ be a $(k, l)$-connected graph. Then

(i) $|V(G)| \geq k+l-1$.

(ii) Suppose that $l^{\prime}>l \geq 2$ and $|V(G)| \geq k+l^{\prime}-1$. If $G$ is $(k, l)$-connected, then $G$ is $\left(k, l^{\prime}\right)$-connected, but cannot be minimally $\left(k, l^{\prime}\right)$-connected.

Proof: (i) Suppose that $|V(G)|<k+l-1$. Let $X \subseteq V(G)$ with $|X|=k-1$. Then $|V(G-X)|<$ $l$, and so $\kappa_{l}(G) \leq k-1$, contrary to the fact that $G$ is $(k, l)$-connected.

(ii) Suppose that $G$ is not $\left(k, l^{\prime}\right)$-connected. Then $\kappa_{l^{\prime}}(G) \leq k-1$, and so there exists $X \subset V(G)$ with $|X| \leq k-1$ such that either $\omega(G-X) \geq l^{\prime}>l$, whence $\kappa_{l}(G) \leq \kappa_{l^{\prime}}(G) \leq k-1$, contrary to $\kappa_{l}(G) \geq k$; or $|V(G-X)| \leq l^{\prime}-1$, whence $|V(G)|<k+l^{\prime}-1$, contrary to the assumption. Hence $\kappa_{l^{\prime}}(G) \geq k$.

To prove that $G$ is not minimally $\left(k, l^{\prime}\right)$-connected, we argue by contradiction and assume that $G$ is minimally $\left(k, l^{\prime}\right)$-connected. Then $\forall e \in E(G), \kappa_{l^{\prime}}(G-e) \leq k-1$. There exists an $X \subset V(G-e)=V(G)$ with $|X| \leq k-1$. If $\omega(G-e-X) \geq l^{\prime}$, then $\omega(G-X) \geq l^{\prime}-1 \geq l$, whence $\kappa_{l}(G) \leq k-1$, contrary to $\kappa_{l}(G) \geq k$. If $|V(G-e-X)| \leq l^{\prime}-1$, then since $|V(G-X)|=$ $|V(G-e-X)|$, we have $|V(G)|<k+l^{\prime}-1$, contrary to $|V(G)| \geq k+l^{\prime}-1$. Thus, $G$ is $\left(k, l^{\prime}\right)$-connected, but not minimally $\left(k, l^{\prime}\right)$-connected.

Suppose that $l \geq 3$ and $H$ is a tree such that there are at least two non-adjacent vertices $u, v \in V(H)$ satisfying $d(u)=d(v)=l-1=\Delta(G)$. Let $\mathscr{T}(l-1)$ be the set of all such trees, and let $\mathscr{T}_{n}(l-1)=\{H \in \mathscr{T}(l-1):|V(H)|=n\}$.

Theorem 4.3.2. Let $G$ be a tree and $l \geq 3$. Then $G$ is minimally $(2, l)$-connected if and only if $G \in \mathscr{T}(l-1)$. 
Proof: First we assume that $G \in \mathscr{T}(l-1)$. Since $\Delta(G)=l-1, \kappa_{l}(G) \geq 2$. To prove that $G$ is minimally $(2, l)$-connected, we need to show that $\forall e \in E(G), \kappa_{l}(G-e) \leq 1$. By assumption, $G$ has at least one vertex $v$ which is not incident with edge $e$, such that $d(v)=l-1$. Since $G$ is a tree, both $\omega(G-v)=l-1$ and each component of $G-v$ is a tree. As $e$ must be in a component of $G-v, \omega(G-e-v)=l$, whence $\kappa_{l}(G-e)=1$.

We now assume that $G$ is minimally $(2, l)$-connected to prove the necessity. Since $G$ is a tree and $\kappa_{l}(G) \geq 2$, we have $\Delta(G) \leq l-1$.

Claim 1: Let $e \in E(G)$. Then $\exists u \in V(G)$ which is not incident with $e$ such that $d(u)=l-1$.

Proof of Claim 1: Since $G$ is minimally $(2, l)$-connected, $\kappa_{l}(G-e)=1$, and so $\exists u \in V(G)$ such that $\omega(G-e-u) \geq l$. Thus $\omega(G-u) \geq l-1$ and $d(u) \geq l-1$. Since $\Delta(G) \leq l-1$, $\Delta(G)=d(u)=l-1$. Note that $u$ is not incident with $e$, as otherwise, $\omega(G-u)=\omega(G-e-u) \geq l$, contrary to the fact that $G$ is $(2, l)$-connected. Thus Claim 1 must hold.

By Claim $1, \Delta(G)=l-1$ and so $\exists u \in V(G), d(u)=l-1$. Let $e^{\prime} \in E(G)$ be an edge incident with $u$. By Claim 1, there exists a vertex $u^{\prime} \in V(G)$ such that $d\left(u^{\prime}\right)=l-1$ and $e^{\prime}$ is not incident with $u^{\prime}$. Thus $u^{\prime} \neq u$. If $u^{\prime}$ is not adjacent to $u$, then the theorem holds. Hence we assume that $e^{\prime \prime}=u u^{\prime} \in E(G)$. By Claim 1, there exists a vertex $u^{\prime \prime} \in V(G)$ such that $d\left(u^{\prime \prime}\right)=l-1$ and $u^{\prime \prime} \notin\left\{u, u^{\prime}\right\}$. Thus $G$ has 3 vertices with degree $l-1$. Since $G$ is a tree, at least 2 of these vertices of degree $l-1$ are non-adjacent. Hence $G \in \mathscr{T}(l-1)$.

Corollary 4.3.3. Let $G$ be a tree. Then $G$ is minimally $(2,3)$-connected if and only if $G$ is a path $P_{n}$ (a path with $n$ vertices), where $n \geq 5$.

Let $G$ be a graph, and $k \geq 1, l \geq 2$ be integers. A $(k, l)$-cut of $G$ is a set $F \subseteq V(G)$ such that $|F|=k$ and $\omega(G-F) \geq l$. As any $(1, l)$-cut consists of a single vertex, a $(1, l)$-cut is also called a $(1, l)$-cut-vertex. We shall use the notation $J^{l}(G)$ to denote the set of all $(1, l)$-cut-vertices of $G$.

Lemma 4.3.4. Let $l \geq 3$. Suppose that $G$ is a connected, minimally $(2, l)$-connected graph. Let $B$ be a cyclic block of $G$. Then $\forall e \in E(B), \exists u \in V(B)$ such that $u \in J^{l-1}(G)$ and such that $u$ is not incident with $e$.

Proof: Since $G$ is minimally $(2, l)$-connected and $e \in E(B) \subseteq E(G), \kappa_{l}(G-e)=1$. Thus $\exists u \in V(G-e)=V(G)$ such that $\omega(G-e-u) \geq l$. Hence $\omega(G-u) \geq l-1$. Since $G$ is $(2, l)$-connected, it must be the case that $\omega(G-u)=l-1$, and so $u$ is a $(1, l-1)$-cut-vertex of graph $G$. We claim that $u \in V(B)$. If not, then $u \notin V(B)=V(B-e)$, and so $B-e$ is contained in a component of $G-e-u$. Hence $\omega(G-u)=\omega((G-e-u)+e)=\omega(G-e-u) \geq l$, contrary to the fact that $G$ is $(2, l)$-connected. We also claim that $u$ is not incident with edge $e$. If not, then $\omega(G-u)=\omega(G-e-u) \geq l$, contrary to the fact that $G$ is $(2, l)$-connected. Thus the lemma must hold. 
Theorem 4.3.5. Let $l \geq 3$. A connected graph $G$ is minimally $(2, l)$-connected if and only if each of the following holds.

(i) Each cut vertex of $G$ has degree no more than $l-1$ in the block-cutvertex graph of $G$.

(ii) If $G$ is a tree, then $G \in \mathscr{T}(l-1)$.

(iii) For each cyclic block $B$ not isomorphic to $K_{3}$ and for each lane $L$ of $B$, if $J\left(B-L_{0}\right)$ denotes the set of all cut vertices of $B-L_{0}$ and $S=V(L) \cap J^{l-1}(G)$, then either $|S| \geq 2$ and $\operatorname{diam}_{L}(S) \geq 2$, or $J\left(B-L_{0}\right) \cap J^{l-1}(G) \neq \emptyset$.

(iv) If a block $B$ of $G$ is isomorphic to $K_{3}$, then $\forall v \in V(B), v \in J^{l-1}(G)$.

Proof: Assume that $G$ is connected and minimally $(2, l)$-connected.

(i) Since $G$ is $(2, l)$-connected, $G$ has no $(1, l)$-cut-vertices. Thus each cut vertex of $G$ has degree at most $l-1$ in the block-cutvertex graph of $G$.

(ii) It follows from Theorem 4.3.2.

(iii) Since $G$ is connected and minimally $(2, l)$-connected, $\forall e \in E(L) \subseteq E(G), \kappa_{l}(G-e)=1$, and so $\exists u \in V(G-e)=V(G)$ such that $\omega(G-e-u) \geq l$. Thus $\omega(G-u) \geq l-1$ and $u$ is a $(1, l-1)$-cut-vertex of $G$. Suppose first that $u \notin V(L)$. If $B-L_{0}$ is contained in a component of $G-u-L$, then $\omega(G-u)=\omega(G-u-L)=\omega(G-u-e) \geq l$, contrary to the fact that $G$ is $(2, l)$-connected. Thus $u$ must be a cut vertex of $B-L_{0}$, and so $J\left(B-L_{0}\right) \cap J^{l-1}(G) \neq \emptyset$, and (iii) holds.

Now assume that $u \in V(L)$. Let $e^{\prime} \in E(L)$ be an edge incident with $u$. By Lemma 5.2.2, $\exists v \in V(B)$ which is not incident with $e^{\prime}$ such that $v \in J^{l-1}(G)$. Thus $v \neq u$. If $v \notin V(L)$, then $J\left(B-L_{0}\right) \cap J^{l-1}(G) \neq \emptyset$, and (iii) holds. Thus we may assume that $v \in V(L)$. If $u$ and $v$ are non-adjacent, then $|S| \geq 2$ and $\operatorname{diam}_{L}(S) \geq 2$, and (iii) holds. If $u$ and $v$ are adjacent in $L$, then let $e^{\prime \prime}=u v$. By Lemma 5.2.2, $\exists x \in V(B)$ such that $x \in J^{l-1}(G)$ and such that $x$ is not incident with $e^{\prime \prime}$. Thus $x \notin\{u, v\}$. If $x \notin V(L)$, then $J\left(B-L_{0}\right) \cap J^{l-1}(G) \neq \emptyset$, and (iii) holds. Hence we assume that $x \in V(L)$. Then $u, v, x \in V(L)$. Now we claim that $L$ is not isomorphic to $K_{3}$. Otherwise, if $L$ is isomorphic to $K_{3}$, and by the definition of a lane, there is at most one vertex in $L$ whose degree is greater than 2 in $B$. If $V(B-L) \neq \emptyset$ then $\kappa(B)=1$, contrary to the fact that $B$ is a cyclic block. if $V(B-L)=\emptyset$, which means $L$ is $B$ itself, contrary to the fact that $B$ is not isomorphic to $K_{3}$. Hence $L$ is not isomorphic to $K_{3}$ and so at least one of vertices $u, v$ is non-adjacent to $x$. Hence (iii) must hold.

(iv) By Lemma 5.2.2, $\forall e \in E\left(K_{3}\right)$, the non-adjacent vertex is in $J^{l-1}(G)$. Thus, $\forall v \in V\left(K_{3}\right)$, $v \in J^{l-1}(G)$.

We now prove the sufficiency. By Theorem 4.3.2, we may assume that $G$ is not a tree. By (i), $G$ has no $(1, l)$-cut-vertices. Thus $\kappa_{l}(G) \geq 2$ and so $G$ is $(2, l)$-connected. We need to prove

$$
\forall e \in E(G), \kappa_{l}(G-e) \leq 1
$$

Pick an edge $e \in E(G)$. There are 3 cases: 
Case 1: The edge $e$ lies in a cyclic block $B$ which is isomorphic to $K_{3}$.

Let $v$ be the vertex in $B$ such that $v$ is not incident with $e$. By (iv), $v$ is a $(1, l-1)$-cut-vertex of $G$. Thus $\omega(G-v) \geq l-1$. Since $B$ is isomorphic to $K_{3}, e$ must be a cut edge of a component $H$ of $G-v$. Hence $\omega(G-e-v) \geq l$, and so $\kappa_{l}(G-e)=1$. Thus (1) holds.

Case 2: Edge $e$ lies in a cyclic block $B$ which is not isomorphic to $K_{3}$.

Let $L$ be the lane in $B$ such that $e \in E(L)$. Then either $J\left(B-L_{0}\right) \cap J^{l-1}(G) \neq \emptyset$, or $|S| \geq 2$ and $\operatorname{diam}_{L}(S) \geq 2$. Assume first that $|S| \geq 2$ and $\operatorname{diam}_{L}(S) \geq 2$. Then $L$ has at least 2 non-adjacent vertices which are $(1, l-1)$-cut-vertices of $G$. Hence there is a vertex $v \in V(L)$ such that $v \in J^{l-1}(G)$ and such that $v$ is not incident with $e$. Thus $\omega(G-v) \geq l-1$. Since $e \in E(L)$ and $L$ is a lane in $B$, by the definition of a lane, $e$ must be a cut edge of a component of $G-v$. Thus $\omega(G-e-u) \geq l$, and so $\kappa_{l}(G-e)=1$. Hence (1) holds. Therefore, by (iii), we assume that $J\left(B-L_{0}\right) \cap J^{l-1}(G) \neq \emptyset$. Let $v \in J\left(B-L_{0}\right) \cap J^{l-1}(G)$. Since $v \in J^{l-1}(G)$, $\omega(G-v) \geq l-1$ and $e$ is in a component $H$ of $G-v$. Let $x$ and $y$ be the end vertices of lane $L$. Since $v$ is a cut vertex of $B-L_{0}, \kappa_{G}(x, y)=2$, whence $e$ is a cut edge of the component $H$ in $G-v$. Then $\omega(G-e-v) \geq l$, whence $\kappa_{l}(G-e)=1$, and so (1) holds.

Case 3: The edge $e$ does not lie in any cyclic block of $G$.

Since $G$ is not a tree, $G$ must have a cyclic block $B$. By (iii) and (iv), whether $B$ is isomorphic to $K_{3}$ or not, $G$ has a $(1, l-1)$-cut-vertex $v$ which is not incident with $e$. Hence $\omega(G-v) \geq l-1$ and $e$ lies in a component $H$ of $G-v$. Since $e$ does not lie in any cyclic block of $G, e$ must be a cut edge of $H$. Thus $\omega(G-e-v) \geq l$, whence $\kappa_{l}(G-e)=1$, and so (1) holds.

Corollary 4.3.6. Let $G$ be a connected, minimally $(2, l)$-connected graph. Then every cyclic block of $G$ is minimally 2-connected.

Proof: Let $B$ be a cyclic block of $G$. By Theorem 4.2.1, to prove $B$ is minimally 2-connected, it suffices to show that each cycle in $B$ has no chords. Assume that there is a cycle $C$ in $B$ with a chord $e=x y$. By the definition of a lane, $e$ is a lane of $B$. By Theorem 4.3 .5 (iii), it must be the case that $J(B-e) \cap J^{l-1}(G) \neq \emptyset$, and let $v \in J(B-e) \cap J^{l-1}(G)$. Since $B$ is 2-connected and $v$ is a cut vertex of $B-e, x$ and $y$ must be in different components of $B-e-v$, whence $\kappa_{B-e}(x, y)=1$. But since $e$ is a chord of cycle $C$ in $B, \kappa_{B-e}(x, y) \geq 2$. We get a contradiction. Hence, every cyclic block of $G$ is minimally 2-connected.

\section{$4.4 \quad F(n, 2, l), f(n, 2, l), \operatorname{Ex}(n, 2, l), \operatorname{Sat}(n, 2, l)$ and $\mathscr{I}(n, 2, l)$}

In this section, we shall determine the value of $F(n, 2, l)$ and $f(n, 2, l)$, and discover the family of $\operatorname{Ex}(n, 2, l), \operatorname{Sat}(n, 2, l)$ and $\mathscr{I}(n, 2, l)$.

Lemma 4.4.1. Let $G$ be a connected, minimally $(2, l)$-connected graph. Let $l \geq 3$ and $|V(G)|=$ $n$. 
(i) If $G$ is acyclic, then $2 l-1 \leq n$;

(ii) If $G$ is cyclic, then $2 l \leq n$.

Proof: (i) By Theorem 4.3.2, there are two non-adjacent vertices $u$ and $v$ such that $d(u)=$ $d(v)=l-1$. Hence $2(l-1)-1+2 \leq n$, that is $2 l-1 \leq n$.

(ii) By Corollary 4.3.6, there must be a cyclic block which is minimally 2-connected. There are two cases here. If the cyclic block is a $K_{3}$, then by Theorem 4.3.5, all the three vertices of $K_{3}$ are $(1, l-1)$-cut-vertices, and hence there are at least $3(l-2)+3$ vertices. Thus $n \geq 3(l-2)+3=$ $3 l-3 \geq 2 l$, since $l \geq 3$. If the cyclic block is not a $K_{3}$, then the block has at least 4 vertices, and by Theorem 4.3.5, at least 2 of them are $(1, l-1)$-cut-vertices. Hence $n \geq 2(l-2)+4$, that is $2 l \leq n$.

Lemma 4.4.2. Let $G$ be a connected, minimally $(2, l)$-connected graph with $|V(G)|=n$ and $|E(G)|=m$. Then

(i) $n-1 \leq m \leq 2 n-2 l$.

(ii) $m=2 n-2 l$ holds if and only if one of the following holds:

(a) $G$ is a tree and $n=2 l-1$; or

(b) $G$ has only one cyclic block, the cyclic block is isomorphic to $K_{2, n-2 l+2}$, and $G$ has exactly two non-adjacent $(1, l-1)$-cut-vertices; or

(c) $l=3, n=6$ and the only cyclic block of $G$ is isomorphic to $K_{3}$.

Proof: If $G$ is a tree, then $m=n-1$. By Lemma 4.4.1, $2 l-1 \leq n$. Hence $m=n-1 \leq$ $n-1+n-(2 l-1) \leq 2 n-2 l$, where equality holds if and only if $n=2 l-1$. Thus the lemma must hold.

Now we assume that $G$ is cyclic. Since $G$ is connected, $m \geq n$. We still need to prove $m \leq 2 n-2 l$. Suppose that $G$ has $t$ cyclic blocks which are not isomorphic to $K_{3}$, denoted by $H_{1}, H_{2}, \ldots, H_{t}$, and $s$ cyclic blocks which are isomorphic to $K_{3}$. Let $n^{\prime}$ be the total number of vertices of all cyclic blocks, ans so $n^{\prime}=3 s+\left(n_{1}+n_{2}+\cdots+n_{t}\right)$. Each $H_{i}$ has $n_{i}$ vertices and $m_{i}$ edges, for $i=1,2, \ldots, t$. By Corollary 4.3.6, each cyclic block is a minimally 2-connected graph. By Theorem 4.2.1, $m_{i} \leq 2 n_{i}-4$ for $i=1,2, \ldots, t$. Then $m=3 s+m_{1}+m_{2}+\cdots+m_{t}+(t+s-$ $1)+n-\left(3 s+n_{1}+n_{2}+\cdots+n_{t}\right) \leq 3 s+\left(n_{1}+n_{2}+\cdots+n_{t}\right)+n-3 t-2 s-1=n^{\prime}+n-3 t-2 s-1$. Let $M=n^{\prime}+n-3 t-2 s-1$. We have the following claim.

Claim: When $M$ reaches the maximum value, there is exactly one cyclic block in the graph.

Proof of the Claim: Without loss of generality, we may assume that $n^{\prime} \geq 4$. If the number of cyclic blocks is 1 , then by Corollary 4.3.6 and Theorem 4.2.1, the maximum value of $M$ is $2 n^{\prime}-4+\left(n-n^{\prime}\right)=n^{\prime}+n-4$. If the number of cyclic blocks is at least 2 , then $t+s \geq 2$. The maximum value of $M$ is $n^{\prime}+n-3 t-2 s-1=n^{\prime}+n-2(t+s)-t-1<n^{\prime}+n-4$. This completes the proof of the claim. 


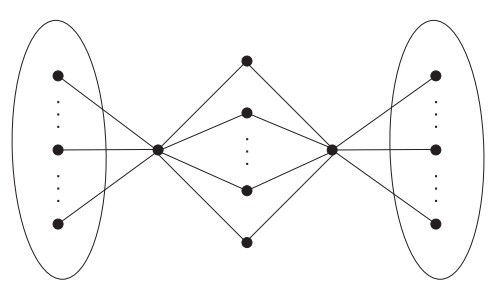

(a) $G_{2, s, t}$

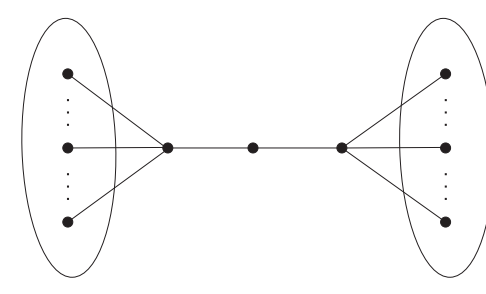

(b) $T_{3, t}$

Figure 4.1: Some classes of graphs

Case 1: $t \neq 0$. By the Claim, when $M$ reaches the maximum value, $t=1, s=0$ and $M=n^{\prime}+n-4=n_{1}+n-4$. By Theorem 4.3.5, there are at least two $(1, l-1)$-cut-vertices in a minimally $(2, l)$-connected graph. Hence $n_{1} \leq n-2(l-2)$. Thus $m \leq 2 n-2 l$, and (i) must hold. The equality holds if and only if $t=1, s=0, n_{1}=n-2(l-2)$ and $m_{1}=2 n_{1}-4$. By Theorem 4.2.1, $m_{1}=2 n_{1}-4$ if and only the cyclic block is isomorphic to $K_{2, n-2 l+2}$. And $n_{1}=n-2(l-2)$ holds if and only if there are exactly two vertices which are not in the cyclic block, i.e., $G$ has exactly two non-adjacent $(1, l-1)$-cut-vertices, by Theorem 4.3.5. Thus (ii) must hold.

Case 2: $t=0$. By the Claim, when $M$ reaches the maximum value, $t=0, s=1$ and $M=n^{\prime}+n-3=n$. By Lemma 4.4.1, $M=n \leq 2 n-2 l$, and the equality holds if and only if $n=2 l$. Since the only cyclic block is a $K_{3}$, by Theorem 4.3 .5 , each vertex of the cyclic block is a $(1, l-1)$-cut-vertex, and thus the number of vertices in the graph is $n=3+3(l-2)=3 l-3$. Hence $M=2 n-2 l$ holds if and only if $n=2 l$ and $n=3 l-3$, i.e., $l=3$ and $n=6$.

Let $K_{2, s}$ be a complete bipartite graph with bipartition $(A, B)$ such that $|A|=2$ and $|B|=s$. Let $G_{2, s, t}$ denote the graph obtained from $K_{2, s}$ by joining each vertex in set $A$ to $t$ new vertices, respectively, as shown in Figure 4.1(a). Let $u$ and $v$ be two non-adjacent vertices of $P_{3}$. Let $T_{3, t}$ denote the graph obtained from $P_{3}$ by joining each of $u, v$ to $t$ new vertices, respectively, as shown in Figure 4.1(b). Graph $G_{3,3}$ is shown in Figure 4.2(a).

Theorem 4.4.3. (i) $F(n, 2, l)=2 n-2 l$.

(ii) $\operatorname{Ex}(5,2,3)=\left\{P_{5}\right\} ; \operatorname{Ex}(6,2,3)=\left\{G_{3,3}, G_{2,2,1}\right\}$; $\operatorname{Ex}(n, 2,3)=\left\{G_{2, n-4,1}\right\}$ for $n \geq 7$.

(iii) When $l \geq 4$ and $n=2 l-1, \operatorname{Ex}(n, 2, l)=\left\{T_{3, l-2}\right\}$.

(iv) When $l \geq 4$ and $n \geq 2 l, E x(n, 2, l)=\left\{G_{2, n-2 l+2, l-2}\right\}$.

Proof: When $l=2$, by Theorem 4.2.1, $F(n, 2,2)=2 n-4$ and $E x(n, 2,2)=\left\{K_{2, n-2}\right\}$. So we assume that $l \geq 3$. By Lemma 4.4.2, $F(n, 2, l) \leq 2 n-2 l$. In order to prove $F(n, 2, l)=2 n-2 l$, it suffices to show that there exists a connected, minimally $(2, l)$-connected graph with $n$ vertices and $2 n-2 l$ edges. When $l=3$, by Lemma 4.4.1, $n \geq 5$ and $G$ is tree if $n=5$. By Corollary 


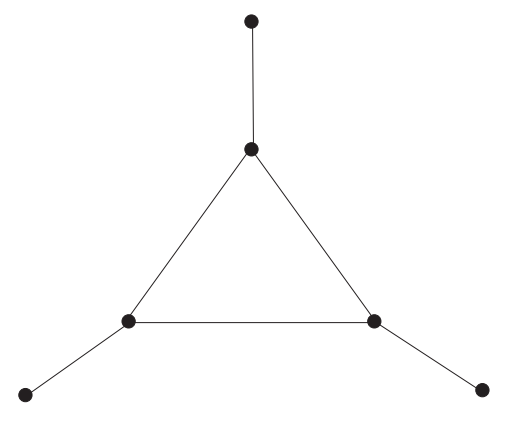

(a) $G_{3,3}$

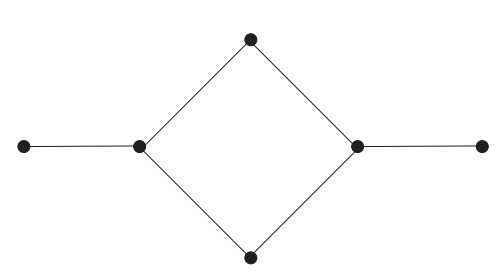

(b) $G_{2,2,1}$

Figure 4.2: Extremal graphs for $F(6,2,3)$

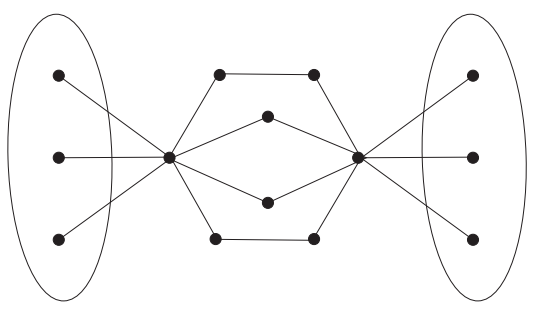

Figure 4.3: An example when $l=5, n=14$ and $m=16$ in the proof of Theorem 4.4.5

4.3.3, $\operatorname{Ex}(5,2,3)=\left\{P_{5}\right\}$. If $n=6, G$ is cyclic and by Lemma 4.4.2, $\operatorname{Ex}(6,2,3)=\left\{G_{3,3}, G_{2,2,1}\right\}$. If $n \geq 7, \forall G \in \operatorname{Ex}(n, 2,3)$, by Lemma 4.4.2, the only cyclic block of $G$ is $K_{2, n-2 l+2}$, and $G$ has exactly two non-adjacent $(1, l-1)$-cut-vertices. Hence, $\operatorname{Ex}(n, 2,3)=\left\{G_{2, n-4,1}\right\}$.

When $l \geq 4$, by Lemma 4.4.1, $n \geq 2 l-1$. If $n=2 l-1$, then $G$ is a tree, and by Theorem 4.3.2, $\forall G \in \operatorname{Ex}(n, 2, l), G \in \mathscr{T}(l-1)$. Then there are two non-adjacent vertices with degree $l-1$. Since $n=2 l-1, G$ must be $T_{3, l-2}$. If $n \geq 2 l$, then by Lemma 4.4.2, $\operatorname{Ex}(n, 2, l)=\left\{G_{2, n-2 l+2, l-2}\right\}$. Thus, the theorem holds.

Theorem 4.4.4. (i) $f(n, 2, l)=n-1$.

(ii) $\operatorname{Sat}(n, 2, l)=\mathscr{T}_{n}(l-1)$.

Proof: By Lemma 4.4.2, $f(n, 2, l) \geq n-1$. In order to prove $f(n, 2, l)=n-1$, it suffices to show that there's a connected, minimally $(2, l)$-connected graph $G$ such that $|V(G)|=n$ and $|E(G)|=n-1$. Graph $g$ must be a tree, since $|E(G)|=|V(G)|-1$. By Theorem 4.3.2, $G \in \mathscr{T}(l-1)$. Thus (i) holds. Since $G$ has $n$ vertices, $\operatorname{Sat}(n, 2, l)=\mathscr{T}_{n}(l-1)$. (ii) must hold.

Theorem 4.4.5. $\mathscr{I}(n, 2, l)=\{i \in \mathbb{N}: n-1 \leq i \leq 2 n-2 l\}$.

Proof: It suffices to show that for each $m \in \mathbb{N} \cap[n-1,2 n-2 l]$, there is a graph $G \in \mathscr{F}(n, 2, l)$ such that $|E(G)|=m$. For each $m$, we will construct a minimally $(2, l)$-connected graph with $n$ vertices and $m$ edges. When $m=n-1, G=P_{n}$. When $n \leq m \leq 2 n-2 l$, we construct a 
minimally $(2, l)$-connected graph $G$ as follows: Let $C$ be a cycle with $2 n-m-2(l-2)$ vertices, and $u_{1}, u_{2}$ are two non-adjacent vertices in $C$. Let $V_{1}$ and $V_{2}$ be two sets of $(l-2)$ vertices, and $V_{1} \cap V_{2}=\emptyset$. Then $G$ is the graph obtained from $C$ by joining $u_{i}$ to each vertex in $V_{i}$ respectively for $\mathrm{i}=1,2$, and joining $u_{1}$ and $u_{2}$ by $m-n$ disjoint paths. These disjoint paths are $m-n$ copies of $P_{3}$. Obviously, $|E(G)|=m$ and $|V(G)|=n$. By Theorem 4.3.5, $G$ is a minimally $(2, l)$-connected graph. An example is shown in Figure 4.3 when $l=5, n=14$ and $m=16$. 


\section{Chapter 5}

\section{Degree sequences and $k$-edge-connected uniform hypergraphs}

\section{$5.1 \quad$ Introduction}

This chapter focuses on the study of degree sequences in hypergraphs.

If a hypergraph $H$ has vertices $v_{1}, v_{2}, \cdots, v_{n}$, then the sequence $\left(d\left(v_{1}\right), d\left(v_{2}\right), \cdots, d\left(v_{n}\right)\right)$ is a degree sequence of $H$. A sequence $d=\left(d_{1}, d_{2}, \cdots, d_{n}\right)$ is hypergraphic if there is a simple hypergraph $H$ with degree sequence $d$, and such a hypergraph $H$ is a realization of $d$, or a $d$-realization. A sequence $d$ is $r$-uniform hypergraphic if there is a simple $r$ uniform hypergraph $H$ with degree sequence $d$. Similarly, a sequence $d$ is multi-hypergraphic if there is a hypergraph (possibly with multiple edges) with degree sequence $d$. A sequence $d$ is $r$-uniform multi-hypergraphic if there is a $r$-uniform hypergraph (possibly with multiple edges) with degree sequence $d$. A 2-uniform hypergraphic sequence is also referred to as a graphic sequence.

Edmonds gave the following characterization for a graphic sequence to have a $k$-edgeconnected realization.

Theorem 5.1.1. (Edmonds [24])

A graphic sequence $d=\left(d_{1}, d_{2}, \cdots, d_{n}\right)$ has a $k$-edge-connected realization if and only if

(i) $d_{i} \geq k$ for $i=1,2, \cdots, n$;

(ii) $\sum_{i=1}^{n} d_{i} \geq 2(n-1)$ if $k=1$.

Characterizations of uniform hypergraphic sequences or uniform multi-hypergraphic sequences to have connected realizations have been obtained by Boonyasombat [7] and Tusyadej, respec- 
tively.

Theorem 5.1.2. (Boonyasombat, Theorem 4.1 of [7])

An $r$-uniform hypergraphic sequence $d=\left(d_{1}, d_{2}, \cdots, d_{n}\right)$ has a connected realization if and only if

(i) $d_{i} \geq 1$ for $i=1,2, \cdots, n$;

(ii) $\sum_{i=1}^{n} d_{i} \geq \frac{r(n-1)}{r-1}$.

Theorem 5.1.3. (Tusyadej, Page 4 of Berge [1])

A nonincreasing integer sequence $d=\left(d_{1}, d_{2}, \cdots, d_{n}\right)$ is the degree sequence of a connected $r$ uniform hypergraph (possibly with multiple edges) if and only if each of the following holds

(i) $\sum_{i=1}^{n} d_{i}$ is a multiple of $r$;

(ii) $d_{n} \geq 1$; and

(iii) $\sum_{i=1}^{n} d_{i} \geq \max \left\{\frac{r(n-1)}{r-1}, r d_{1}\right\}$.

Degree sequence problems of hypergraphs are much harder than those of graphs. Actually the characterizations of hypergraphic sequences is still open for $r \geq 3$ (see $[1,2,20,22,26]$ ). The problem seems to be difficult even for $r=3$. In [15], only the necessary condition for a hypergraphic sequence was given for $r=3$. In fact, in [20], the authors reported that they were neither able to give a polynomial time algorithm nor able to prove that the problem is NP-complete even for $r=3$.

In this paper, we investigate necessary and sufficient conditions for an $r$-uniform hypergraphic sequence to have a $k$-edge-connected realization. Our main results, Theorem 5.1.4 and Theorem 5.1.5 below, generalize Theorems 5.1.1, 5.1.2 and Theorem 5.1.3, respectively.

Theorem 5.1.4. An $r$-uniform hypergraphic sequence $d=\left(d_{1}, d_{2}, \cdots, d_{n}\right)$ has a $k$-edge-connected realization if and only if

(i) $d_{i} \geq k$ for $i=1,2, \cdots, n$;

(ii) $\sum_{i=1}^{n} d_{i} \geq \frac{r(n-1)}{r-1}$ if $k=1$.

Theorem 5.1.5. A nonincreasing integer sequence $d=\left(d_{1}, d_{2}, \cdots, d_{n}\right)$ is the degree sequence of a $k$-edge-connected $r$-uniform hypergraph (possibly with multiple edges) if and only if each of the following holds

(i) $\sum_{i=1}^{n} d_{i}$ is a multiple of $r$;

(ii) $d_{n} \geq k$; and

(iii) $\sum_{i=1}^{n} d_{i} \geq \max \left\{\frac{r(n-1)}{r-1}, r d_{1}\right\}$.

In Section 2 and Section 3, we will present the proofs of Theorem 5.1.4 and Theorem 5.1.5 respectively. A further conjecture will be proposed in Section 4. 


\subsection{The Proof of Theorem 5.1.4}

The main effort will be the proof for the sufficiency. We will first show that $d$ has an $h$-edge connected realization $H$ for some $h \geq 1$. If $h<k$, then we will show that it is possible to perform some edge switching to find a $d$-realization with higher edge connectivity.

The following lemmas hold for any possibly nonsimple hypergraph.

Lemma 5.2.1. Let $H$ be an $r$-uniform hypergraph on $n$ vertices. If $H$ is connected, then $|\mathcal{E}(H)| \geq \frac{n-1}{r-1}$. Moreover, the equality holds if and only if for any edge $E \in \mathcal{E}(H), H-E$ has $r$ components.

Proof: We establish the inequality by induction on $n$. If $n=r$, then it has an edge containing all vertices and so $|\mathcal{E}(H)| \geq 1(|\mathcal{E}(H)|=1$ for simple hypergraphs). Assume that $n \geq r+1$ and that the inequality holds for smaller values of $n$. We remove edges from $H$ one by one until there are at least 2 components. Let $H_{1}, H_{2}, \cdots, H_{t}$ be these components. Removing a single edge can only create at most $r$ components, thus $2 \leq t \leq r$. Suppose that the number of vertices in $H_{i}$ is $n_{i}$ for $1 \leq i \leq t$. Then $\sum_{i=1}^{t} n_{i}=n$. By the inductive hypothesis, $\left|\mathcal{E}\left(H_{i}\right)\right| \geq \frac{n_{i}-1}{r-1}$. Thus $|\mathcal{E}(H)| \geq \sum_{i=1}^{t}\left|\mathcal{E}\left(H_{i}\right)\right|+1=\frac{n-t}{r-1}+1 \geq \frac{n-r}{r-1}+1=\frac{n-1}{r-1}$.

Now suppose that the equality holds. If there exists an edge $E_{0} \in \mathcal{E}(H)$ such that $H-E_{0}$ has less than $r$ components, denoted by $H_{1}, H_{2}, \cdots, H_{t}$, where $1 \leq t<r$. Let $n_{i}$ be the number of vertices in $H_{i}$ for $1 \leq i \leq t$. Then $\sum_{i=1}^{t} n_{i}=n$. Since each $H_{i}$ is a connected $r$-uniform hypergraph, $\left|\mathcal{E}\left(H_{i}\right)\right|=\frac{n_{i}-1}{r-1}$. Then $|\mathcal{E}(H)|=\sum_{i=1}^{t}\left|\mathcal{E}\left(H_{i}\right)\right|+1=\frac{n-t}{r-1}+1>\frac{n-r}{r-1}+1=\frac{n-1}{r-1}$, contrary to $|\mathcal{E}(H)|=\frac{n-1}{r-1}$. Hence for any edge $E \in \mathcal{E}(H), H-E$ has $r$ components.

To prove the sufficiency of the second part, we argue by induction on $n$. If $n=r$, then $|\mathcal{E}(H)|=1=\frac{n-1}{r-1}$, and so we assume that $n>r$ and it holds for smaller values of $n$. Pick $E \in \mathcal{E}(H)$. Let $H_{1}, H_{2}, \cdots, H_{r}$ be the components of $H-E$ and $n_{i}=\left|V\left(H_{i}\right)\right|$ for $i=1,2, \cdots, r$. We claim that for each $i$ and any edge $E^{\prime} \in \mathcal{E}\left(H_{i}\right), H_{i}-E^{\prime}$ has $r$ components. If not, then there exist $j$ with $1 \leq j \leq r$ and an edge $E^{\prime \prime} \in \mathcal{E}\left(H_{j}\right)$ such that $H_{j}-E^{\prime \prime}$ has less than $r$ components. Then $H-E^{\prime \prime}=\left(H_{j}-E^{\prime \prime}\right) \cup\left(\cup_{i \neq j} H_{i}\right)+\{E\}$ has less than $r$ components, contrary to the assumption. Hence the claim holds and by induction, $\left|\mathcal{E}\left(H_{i}\right)\right|=\frac{n_{i}-1}{r-1}$. Thus $|\mathcal{E}(H)|=\sum_{i=1}^{r}\left|\mathcal{E}\left(H_{i}\right)\right|+1=\frac{n-r}{r-1}+1=\frac{n-1}{r-1}$, completing the proof.

Lemma 5.2.2. Let $H$ be an r-uniform h-edge-connected hypergraph and $[X, \bar{X}]$ be an edge-cut of size $h$. Then for any vertex $u \in X$ with $d_{H}(u)>h$ and for any vertex $v \in \bar{X}$, there exist vertices $u_{2}, u_{3}, \cdots, u_{r} \in X$ such that $\left\{u, u_{2}, \cdots, u_{r}\right\} \in \mathcal{E}(H)$ and $\left\{v, u_{2}, \cdots, u_{r}\right\} \notin \mathcal{E}(H)$.

Proof: Let $d_{H}(u)=k$ and $k^{\prime}$ be the number of $(X, \bar{X})$-crossing edges containing $u$. Then $k^{\prime} \leq h<k$, and there are $k-k^{\prime}$ exact- $X$-crossing edges containing $u$. That is, there exist distinct $(r-1)$-subsets $U_{1}, U_{2}, \cdots, U_{\left(k-k^{\prime}\right)}$ of $X$ such that for each $i=1,2, \cdots, k-k^{\prime}, U_{i} \cup\{u\} \in \mathcal{E}(H)$. 
Let $v$ be any vertex in $\bar{X}$. If for each $i=1,2, \cdots, k-k^{\prime}, U_{i} \cup\{v\} \in \mathcal{E}(H)$, then $|[X, \bar{X}]| \geq$ $k^{\prime}+\left(k-k^{\prime}\right)>h$, contrary to $|[X, \bar{X}]|=h$. Thus there exists a set $U_{j}$ where $1 \leq j \leq k-k^{\prime}$ such that $U_{j} \cup\{v\} \notin \mathcal{E}(H)$. Let $U_{j}=\left\{u_{2}, u_{3}, \cdots, u_{r}\right\}$. Then $\left\{u, u_{2}, \cdots, u_{r}\right\} \in \mathcal{E}(H)$ but $\left\{v, u_{2}, \cdots, u_{r}\right\} \notin \mathcal{E}(H)$.

Lemma 5.2.3. Let $d$ be a sequence satisfying Theorem 5.1.4 (i) and (ii). Then for any disconnected d-realization $H$ with components $H_{1}, H_{2}, \cdots, H_{l}$, there exists an edge $E \in \mathcal{E}\left(H_{j}\right)$ such that the number of components of $H_{j}-E$ is at most $r-1$, for some $j$ with $1 \leq j \leq l$.

Proof: Suppose that there is no such edge $E \in \mathcal{E}\left(H_{i}\right)$ for $i=1,2, \cdots, r$. Let $|V(H)|=n$ and $\left|V\left(H_{i}\right)\right|=n_{i}$ for each $i=1,2, \cdots, l$. By Lemma 5.2.1, $\left|\mathcal{E}\left(H_{i}\right)\right|=\frac{n_{i}-1}{r-1}$. Thus $|\mathcal{E}(H)|=$ $\sum_{i=1}^{l}\left|\mathcal{E}\left(H_{i}\right)\right|=\frac{n_{1}+n_{2}+\cdots+n_{r}-l}{r-1}=\frac{n-l}{r-1}<\frac{n-1}{r-1}$, and so $\sum_{i=1}^{n} d_{i}=r|\mathcal{E}(H)|<\frac{r(n-1)}{r-1}$, contrary to Theorem 5.1.4 (ii).

Lemma 5.2.4. Suppose that $H$ is an r-uniform hypergraph with edges $E_{0}=\left\{u, x_{2}, x_{3}, \cdots, x_{r}\right\}$ and $F_{0}=\left\{v, y_{2}, y_{3}, \cdots, y_{r}\right\}$. Let $H^{\prime}$ be a hypergraph obtained from $H$ by deleting edges $E_{0}$ and $F_{0}$, and adding edges $\left\{v, x_{2}, x_{3}, \cdots, x_{r}\right\}$ and $\left\{u, y_{2}, y_{3}, \cdots, y_{r}\right\}$. Let $Z$ be a nonempty proper subset of $V(H)$. If $d_{H^{\prime}}(Z)<d_{H}(Z)$, then one of the following must hold.

(i) $u, y_{2}, y_{3}, \cdots, y_{r} \in Z, v \in \bar{Z}$ and at least one of $x_{2}, x_{3}, \cdots, x_{r}$ is in $\bar{Z}$;

(ii) $u, y_{2}, y_{3}, \cdots, y_{r} \in \bar{Z}, v \in Z$ and at least one of $x_{2}, x_{3}, \cdots, x_{r}$ is in $Z$;

(iii) $v, x_{2}, x_{3}, \cdots, x_{r} \in Z, u \in \bar{Z}$ and at least one of $y_{2}, y_{3}, \cdots, y_{r}$ is in $\bar{Z}$;

(iv) $v, x_{2}, x_{3}, \cdots, x_{r} \in \bar{Z}, u \in Z$ and at least one of $y_{2}, y_{3}, \cdots, y_{r}$ is in $Z$.

Proof: By symmetry, it suffices to show one of the cases. Since $d_{H^{\prime}}(Z)<d_{H}(Z)$, at least one of the two new edges of $H^{\prime}$ is not $(Z, \bar{Z})$-crossing. Without loss of generality, we may assume that $u, y_{2}, y_{3}, \cdots, y_{r} \in Z$. Then $v \in \bar{Z}$, otherwise, $F_{0}$ is not $(Z, \bar{Z})$-crossing in $H$, and thus removing $F_{0}$ will not decrease the number of $(Z, \bar{Z})$-crossing edges, contrary to $d_{H^{\prime}}(Z)<d_{H}(Z)$. Similarly, if $x_{2}, x_{3}, \cdots, x_{r} \in Z$, then $E_{0}$ is not $(Z, \bar{Z})$-crossing in $H$ and thus removing $E_{0}$ will not decrease the number of $(Z, \bar{Z})$-crossing edges, contrary to $d_{H^{\prime}}(Z)<d_{H}(Z)$. Thus at least one of $x_{2}, x_{3}, \cdots, x_{r}$ is in $\bar{Z}$, completing the proof of (i).

Let $h$ be a positive integer, an $h$-minimal set of a hypergraph $H$ is a nonempty proper subset $X$ of $V(H)$ with $d_{H}(X)=h$ such that for any nonempty proper subset $X^{\prime}$ of $X, d_{H}\left(X^{\prime}\right)>h$. By definition, if $H$ is $h$-edge-connected, then any subset $S \subseteq V(H)$ with $d_{H}(S)=h$ contains an $h$-minimal set of $H$.

Lemma 5.2.5. Suppose that $X$ is an h-minimal set of an $r$-uniform hypergraph $H$. Let $X_{1}$ and $X_{2}$ be nonempty proper subsets of $X$ with $X_{1} \cup X_{2}=X$. Then each of the following statements holds. 
(i) $\left|\mathcal{E}_{X_{1} X_{2}}^{H}\right| \geq\left|\mathcal{E}_{X_{1} \bar{X}}^{H}\right|+1$ and $\left|\mathcal{E}_{X_{1} X_{2}}^{H}\right| \geq\left|\mathcal{E}_{X_{2} \bar{X}}^{H}\right|+1$.

(ii) $\left|\mathcal{E}_{X_{1} X_{2}}^{H}\right| \geq \frac{h}{2}-\frac{\left|\mathcal{E}_{X_{1} X_{2} \bar{X}}^{H}\right|}{2}+1$.

Proof: (i) Since $X$ is an $h$-minimal set of $H, d_{H}(X)=\left|\mathcal{E}_{X_{1} \bar{X}}^{H}\right|+\left|\mathcal{E}_{X_{2} \bar{X}}^{H}\right|+\left|\mathcal{E}_{X_{1} X_{2} \bar{X}}^{H}\right|=h$ and $d_{H}\left(X_{1}\right)=\left|\mathcal{E}_{X_{1} \bar{X}}^{H}\right|+\left|\mathcal{E}_{X_{1} X_{2}}^{H}\right|+\left|\mathcal{E}_{X_{1} X_{2} \bar{X}}^{H}\right| \geq h+1$. Thus $\left|\mathcal{E}_{X_{1} X_{2}}^{H}\right| \geq\left|\mathcal{E}_{X_{2} \bar{X}}^{H}\right|+1$. By symmetry, $\left|\mathcal{E}_{X_{1} X_{2}}^{H}\right| \geq\left|\mathcal{E}_{X_{1} \bar{X}}^{H}\right|+1$.

(ii) By (i), $2\left|\mathcal{E}_{X_{1} X_{2}}^{H}\right|+\left|\mathcal{E}_{X_{1} X_{2} \bar{X}}^{H}\right| \geq\left|\mathcal{E}_{X_{1} \bar{X}}^{H}\right|+1+\left|\mathcal{E}_{X_{2} \bar{X}}^{H}\right|+1+\left|\mathcal{E}_{X_{1} X_{2} \bar{X}}^{H}\right|=h+2$. Thus $\left|\mathcal{E}_{X_{1} X_{2}}^{H}\right| \geq$ $\frac{h}{2}-\frac{\left|\mathcal{E}_{X_{1} X_{2} \bar{X}}^{H}\right|}{2}+1$.

Suppose that $[Z, \bar{Z}]$ is an edge-cut of a hypergraph $H$. Let $X_{1}, Y_{1} \subseteq Z$ with $X_{1} \cap Y_{1}=\emptyset$ and $X_{2}, Y_{2} \subseteq \bar{Z}$ with $X_{2} \cap Y_{2}=\emptyset$. Let $\mathcal{E}_{O}^{H}$ be the set of all other edges of $[Z, \bar{Z}]$ which are not in $\mathcal{E}_{X_{1} X_{2}}^{H}$ and $\mathcal{E}_{Y_{1} Y_{2}}^{H^{\prime}}$. Then

$$
d_{H}(Z)=\left|\mathcal{E}_{X_{1} X_{2}}^{H}\right|+\left|\mathcal{E}_{Y_{1} Y_{2}}^{H}\right|+\left|\mathcal{E}_{O}^{H}\right|
$$

Now we are ready to prove Theorem 5.1.4.

Proof of Theorem 5.1.4: Suppose that $d$ has a $k$-edge-connected $r$-uniform realization $H$. For any vertex $v \in V(H)$ whose degree is $d_{i}, d_{i}=|[\{v\}, V-\{v\}]| \geq k$, for $i=1,2, \cdots, n$. When $k=1$, by Lemma $5.2 .1,|\mathcal{E}(H)| \geq\left\lceil\frac{n-1}{r-1}\right\rceil$, and so $\sum_{i=1}^{n} d_{i} \geq \frac{r(n-1)}{r-1}$.

To prove the sufficiency, let $h$ be the maximum edge connectivity among all $d$-realizations. By contradiction, we assume that

$$
h<k \text {. }
$$

First we prove that $h \geq 1$ by showing that $d$ has a simple connected $r$-uniform realization. Let $H$ be a simple $r$-uniform $d$-realization with $l$ components such that

$$
l \text { is minimized. }
$$

If $l=1$, then $H$ is connected, and we are done. Hence we may assume that $l \geq 2$ and let $H_{1}, H_{2}, \cdots, H_{l}$ be the components of $H$.

By Lemma 5.2.3, we may assume that $H_{1}$ has an edge $E=\left\{u_{1}, u_{2}, \cdots, u_{r}\right\}$ such that $H_{1}-E$ has a component $U$ with $u_{1}, u_{2} \in V(U)$. Let $E^{\prime}=\left\{v_{1}, v_{2}, \cdots, v_{r}\right\} \in \mathcal{E}\left(H_{i}\right)$ for some $i$ with $i>1$. Let $G$ be a hypergraph obtained from $H$ by deleting edges $E$ and $E^{\prime}$, and adding edges $\left\{v_{1}, u_{2}, u_{3}, \cdots, u_{r}\right\}$ and $\left\{u_{1}, v_{2}, v_{3}, \cdots, v_{r}\right\}$, as shown in Figure 5.1. Then $V\left(H_{i}\right)$ and $V\left(H_{1}\right)$ are in the same component of $G$, and for each $j$ with $1 \leq j \leq l$, vertices in $V\left(H_{j}\right)$ are in the same component of $G$. Thus the number of components of $G$ is at most $l-1$, contrary to (5.3). therefore there exists a connected $r$-uniform $d$-realization, and so $h \geq 1$. 


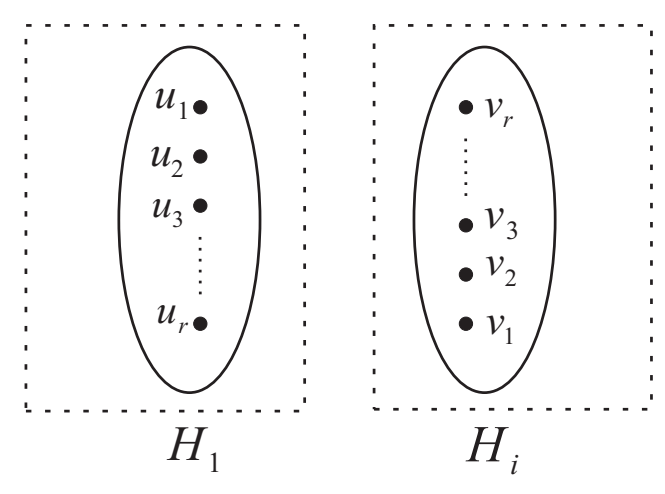

(a) $H$

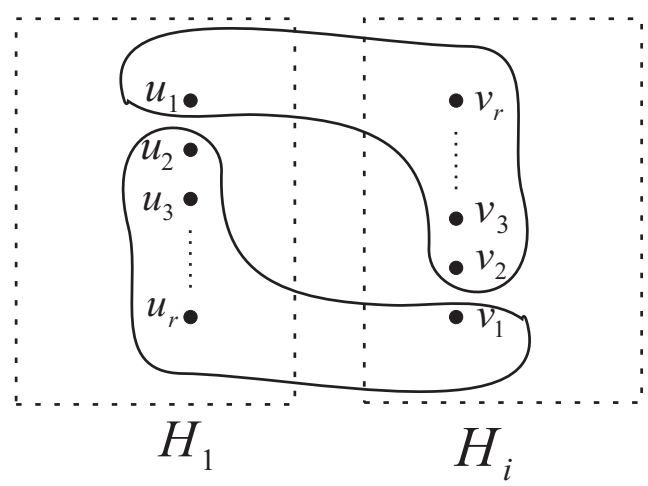

(b) $G$

Figure 5.1: The construction of $G$ from $H$

Let $H$ be an $r$-uniform $d$-realization with edge connectivity $h$ and

with fewest number of edge-cuts of size $h$.

Let $X$ be an $h$-minimal set of $H$. Since $d_{H}(\bar{X})=h, \bar{X}$ must contain an $h$-minimal set, denoted by $Y$. Since $H$ is connected, there exist $u \in X, v \in Y$ and a path $P=$ $\left(u, F_{1}, w_{1}, F_{2}, w_{2}, \cdots, F_{t}, v\right)$ such that

$F_{1}$ is $(X, \bar{X})$-crossing and $F_{t}$ is $(Y, \bar{Y})$-crossing.

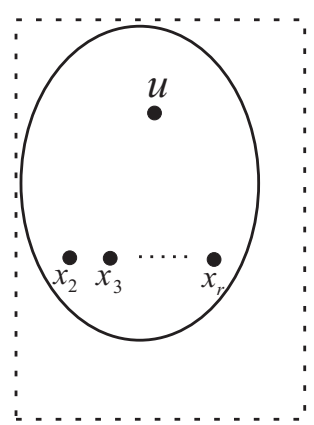

$X$

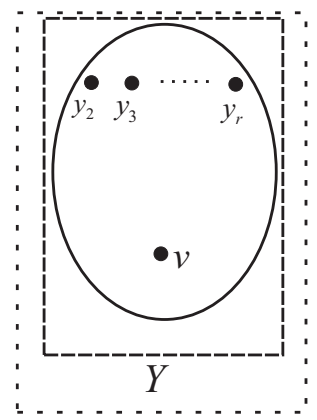

$\bar{X}$

(a) $H$

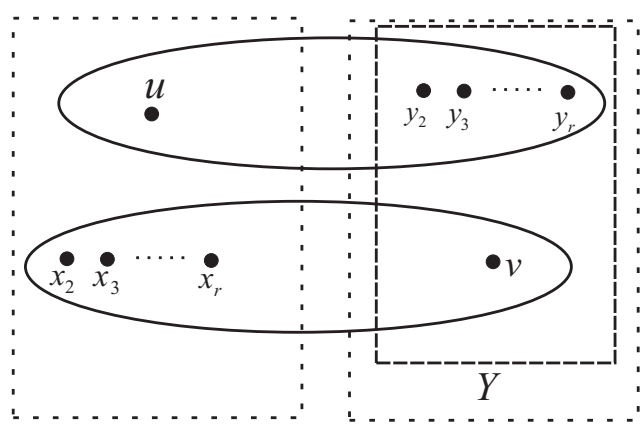

$X$

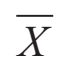

(b) $H^{\prime}$

Figure 5.2: The construction of $H^{\prime}$ from $H$

By Theorem 5.1.4 (i), $d_{H}(u) \geq k>h=|[X, \bar{X}]|$. Then by Lemma 5.2.2, there exist vertices $x_{2}, x_{3}, \cdots, x_{r} \in X$ such that $E_{1}=\left\{u, x_{2}, x_{3}, \cdots, x_{r}\right\} \in \mathcal{E}(H)$ but $\left\{v, x_{2}, x_{3}, \cdots, x_{r}\right\} \notin$ $\mathcal{E}(H)$. Similarly, there exist $y_{2}, y_{3}, \cdots, y_{r} \in Y$ such that $E_{2}=\left\{v, y_{2}, y_{3}, \cdots, y_{r}\right\} \in \mathcal{E}(H)$ but $\left\{u, y_{2}, y_{3}, \cdots, y_{r}\right\} \notin \mathcal{E}(H)$. Let $H^{\prime}$ be the hypergraph obtained from $H$ by deleting edges 
$E_{1}$ and $E_{2}$, and by adding edges $E_{1}^{\prime}=\left\{v, x_{2}, x_{3}, \cdots, x_{r}\right\}$ and $E_{2}^{\prime}=\left\{u, y_{2}, y_{3}, \cdots, y_{r}\right\}$, as shown in Figure 5.2. Then $d_{H^{\prime}}(X)=h+2$ and $d_{H^{\prime}}(Y)=h+2$. By the definition of $H^{\prime}$, $\mathcal{E}\left(H^{\prime}\right)=\left(\mathcal{E}(H)-\left\{E_{1}, E_{2}\right\}\right) \cup\left\{E_{1}^{\prime}, E_{2}^{\prime}\right\}$. An edge-cut is new if it is not an edge-cut of $H$.

Claim 1: If $H^{\prime}$ has a new edge-cut $[Z, \bar{Z}]$ of size at most $h$, then each of the following holds.

(i) $H$ has an $(X \cap Z, X \cap \bar{Z}, Y \cap Z, Y \cap \bar{Z})$-crossing edge.

(ii) $H$ has no edges crossing exactly three of $X \cap Z, X \cap \bar{Z}, Y \cap Z$ and $Y \cap \bar{Z}$.

Proof of Claim 1: Suppose that $H^{\prime}$ introduces a new edge-cut $[Z, \bar{Z}]$ with size $\leq h$. Then $d_{H^{\prime}}(Z) \leq h<d_{H}(Z)$. By Lemma 5.2.4 and by symmetry, we may assume that $u, y_{2} \in Z$ and $v, x_{2} \in \bar{Z}$, as shown in Figure 5.3.

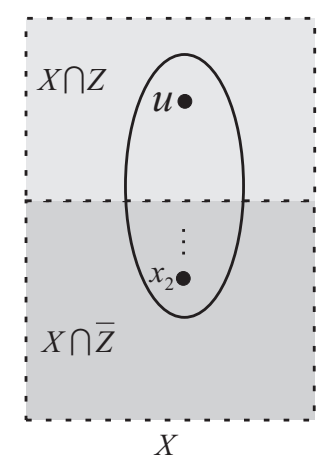

(a) $H$

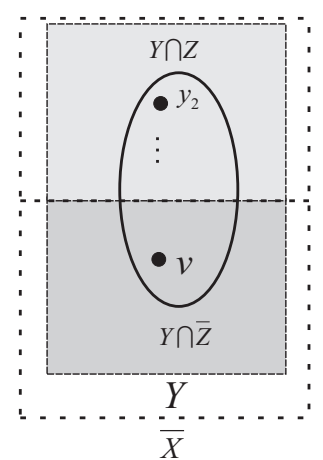

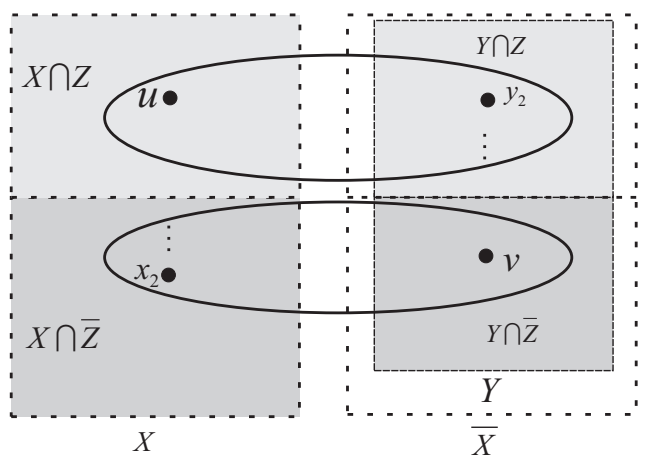

(b) $H^{\prime}$

Figure 5.3: New edge-cut $[Z, \bar{Z}]$ in $H^{\prime}$

Let $X \cap Z=X_{1}, X \cap \bar{Z}=X_{2}, Y \cap Z=Y_{1}$ and $Y \cap \bar{Z}=Y_{2}$. By Lemma 5.2.5, $\left|\mathcal{E}_{X_{1} X_{2}}^{H}\right| \geq$ $\frac{h}{2}-\frac{\left|\mathcal{E}_{X_{1} X_{2} \bar{X}}^{H}\right|}{2}+1$ and $\left|\mathcal{E}_{Y_{1} Y_{2}}^{H}\right| \geq \frac{h}{2}-\frac{\left|\mathcal{E}_{Y_{1} Y_{2} \bar{Y}}^{H}\right|}{2}+1$. By the construction of $H^{\prime}$ from $H$, we have $\left|\mathcal{E}_{X_{1} X_{2}}^{H^{\prime}}\right|=\left|\mathcal{E}_{X_{1} X_{2}}^{H}\right|-1$ and $\left|\mathcal{E}_{Y_{1} Y_{2}}^{H^{\prime}}\right|=\left|\mathcal{E}_{Y_{1} Y_{2}}^{H}\right|-1$. By (5.1),

$$
\begin{aligned}
d_{H^{\prime}}(Z) & =\left|\mathcal{E}_{X_{1} X_{2}}^{H^{\prime}}\right|+\left|\mathcal{E}_{Y_{1} Y_{2}}^{H^{\prime}}\right|+\left|\mathcal{E}_{O}^{H^{\prime}}\right| \\
& =\left|\mathcal{E}_{X_{1} X_{2}}^{H}\right|+\left|\mathcal{E}_{Y_{1} Y_{2}}^{H}\right|+\left|\mathcal{E}_{O}^{H^{\prime}}\right|-2 \\
& \geq h+\left|\mathcal{E}_{O}^{H^{\prime}}\right|-\frac{\left|\mathcal{E}_{X_{1} X_{2} \bar{X}}^{H}\right|}{2}-\frac{\left|\mathcal{E}_{Y_{1} Y_{2} \bar{Y}}^{H}\right|}{2} \\
& =h+\frac{\left|\mathcal{E}_{O}^{H^{\prime}}\right|-\left|\mathcal{E}_{X_{1} X_{2} \bar{X}}^{H}\right|}{2}+\frac{\left|\mathcal{E}_{O}^{H^{\prime}}\right|-\left|\mathcal{E}_{Y_{1} Y_{2} \bar{Y}}^{H}\right|}{2} .
\end{aligned}
$$

By (5.5), there must be an edge in $\mathcal{E}_{O}^{H^{\prime}}$ contained in the path $P$ and so $\mathcal{E}_{O}^{H^{\prime}} \neq \emptyset$. Since $\mathcal{E}_{X_{1} X_{2} \bar{X}}$ and $\mathcal{E}_{Y_{1} Y_{2} \bar{Y}}^{H}$ are subsets of $\mathcal{E}_{O}^{H^{\prime}}$, if one of them is a proper subset of $\mathcal{E}_{O}^{H^{\prime}}$, then $d_{H^{\prime}}(Z)>h$, contrary to $d_{H^{\prime}}(Z) \leq h$. Thus $\mathcal{E}_{X_{1} X_{2} \bar{X}}^{H}=\mathcal{E}_{Y_{1} Y_{2} \bar{Y}}^{H}=\mathcal{E}_{O}^{H^{\prime}} \neq \emptyset$. By the definitions of $\mathcal{E}_{X_{1} X_{2} \bar{X}}^{H}$ and $\mathcal{E}_{Y_{1} Y_{2} \bar{Y}}^{H}$, there exists an $(X \cap Z, X \cap \bar{Z}, Y \cap Z, Y \cap \bar{Z})$-crossing edge, and there are no edges crossing exactly three of $X \cap Z, X \cap \bar{Z}, Y \cap Z, Y \cap \bar{Z}$. This completes the proof of Claim 1. 
Since $[X, \bar{X}]_{H^{\prime}}$ is no longer an edge-cut of size $h$ in $H^{\prime}$, if there is not a new edge-cut with size at most $h$ in $H^{\prime}$, then the number of edge-cuts with size $h$ of $H^{\prime}$ is less than that of $H$, contrary to (5.4). Thus, we may assume that $H^{\prime}$ has a new edge-cut $[Z, \bar{Z}]_{H^{\prime}}$ with size at most $h$. By Claim 1 , there is an edge $E_{0}=\left\{a_{1}, a_{2}, \cdots, a_{r}\right\} \in \mathcal{E}(H)$ which is $(X \cap Z, X \cap \bar{Z}, Y \cap Z, Y \cap \bar{Z})$-crossing with minimized $\left|E_{0} \cap X\right|$. (Notice that if $r=3$, then $H$ can never have such an edge, contrary to Claim 1. Hence we may assume that, in the rest of the proof, $r \geq 4$.)

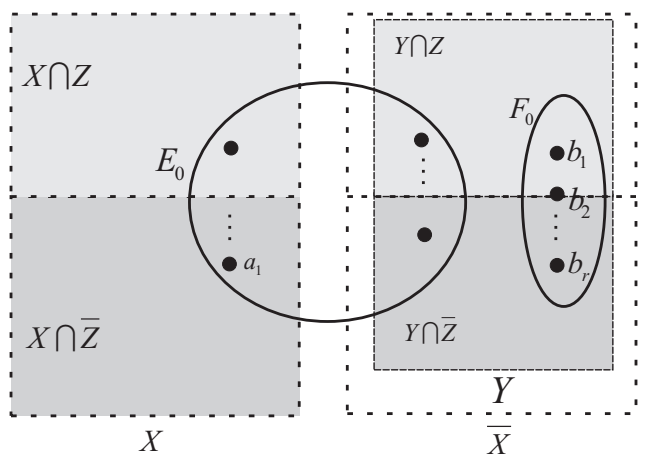

(a) $H$

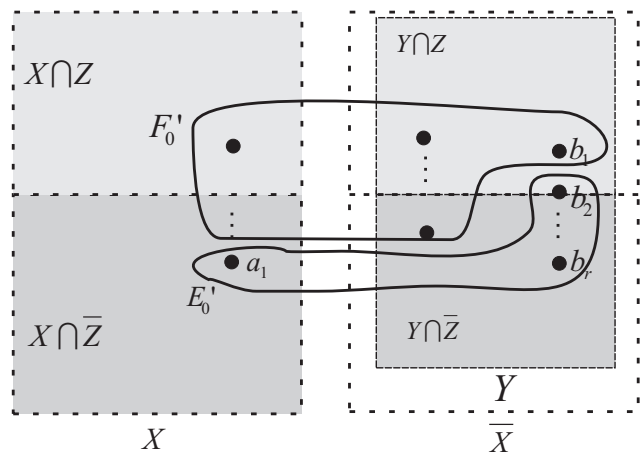

(b) $H^{\prime \prime}$

Figure 5.4: The construction of $H^{\prime \prime}$ from $H$

Denote $E_{0} \cap X=\left\{a_{1}, a_{2}, \cdots, a_{s}\right\}$, where $2 \leq s \leq r-2$. As $Y \backslash E_{0} \neq \emptyset$, let $b_{1} \in Y \backslash E_{0}$. Since $d_{H}\left(b_{1}\right) \geq k>h$, by Lemma 5.2 .2 , there exist vertices $b_{2}, b_{3}, \cdots, b_{r} \in Y$ such that $F_{0}=$ $\left\{b_{1}, b_{2}, \cdots, b_{r}\right\} \in \mathcal{E}(H)$ but $E_{0}^{\prime}=\left\{a_{1}, b_{2}, b_{3}, \cdots, b_{r}\right\} \notin \mathcal{E}(H)$. See Figure 5.4(a).

If $F_{0}^{\prime}=\left\{b_{1}, a_{2}, \cdots, a_{r}\right\} \in \mathcal{E}(H)$, then $F_{0}^{\prime}$ crosses at least three of $X \cap Z, X \cap \bar{Z}, Y \cap Z, Y \cap \bar{Z}$. By Claim 1(ii), $F_{0}^{\prime}$ is $(X \cap Z, X \cap \bar{Z}, Y \cap Z, Y \cap \bar{Z})$-crossing, contrary to the minimality of $\left|E_{0} \cap X\right|$. Thus $F_{0}^{\prime}=\left\{b_{1}, a_{2}, \cdots, a_{r}\right\} \notin \mathcal{E}(H)$. Let $H^{\prime \prime}$ be the hypergraph obtained from $H$ by replacing $E_{0}$ and $F_{0}$ by $E_{0}^{\prime}$ and $F_{0}^{\prime}$, as shown in Figure 5.4(b).

Claim 2: $H^{\prime \prime}$ does not have any new edge-cut of size at most $h$.

Proof of Claim 2: Suppose that there is a new edge-cut $[D, \bar{D}]$ of $H^{\prime \prime}$ with size at most $h$. Then $d_{H^{\prime \prime}}(D) \leq h<d_{H}(D)$. By Lemma 5.2 .4 and by symmetry, we may assume that $a_{1} \in D$ and $b_{1} \in \bar{D}$, as depicted in Figure 5.5.

Let $X \cap D=X_{3}, X \cap \bar{D}=X_{4}, Y \cap D=Y_{3}$ and $Y \cap \bar{D}=Y_{4}$. By Lemma 5.2.5, $\left|\mathcal{E}_{X_{3} X_{4}}^{H}\right| \geq$ $\frac{h}{2}-\frac{\left|\mathcal{E}_{X_{3} X_{4} \bar{X}}^{H}\right|}{2}+1$ and $\left|\mathcal{E}_{Y_{3} Y_{4}}^{H}\right| \geq \frac{h}{2}-\frac{\left|\mathcal{E}_{Y_{3} Y_{4} \bar{Y}}^{H}\right|}{2}+1$. By the construction of $H^{\prime \prime}$ from $H$, we have 


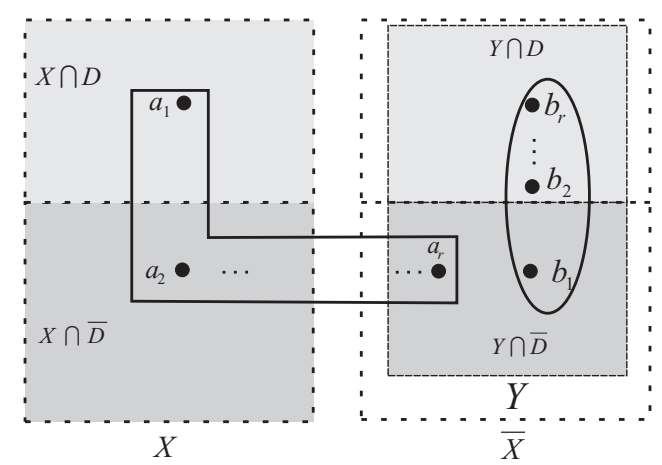

(a) $H$

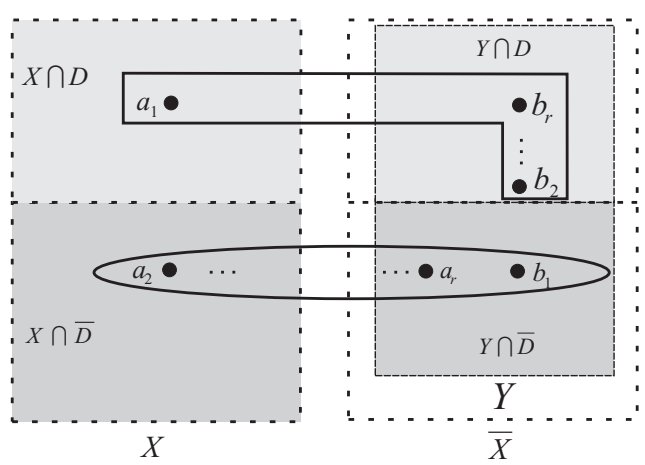

(b) $H^{\prime \prime}$

Figure 5.5: New edge-cut $[D, \bar{D}]$ in $H^{\prime \prime}$

$$
\begin{aligned}
& \left|\mathcal{E}_{X_{3} X_{4}}^{H^{\prime \prime}}\right|=\left|\mathcal{E}_{X_{3} X_{4}}^{H}\right| \text { and }\left|\mathcal{E}_{Y_{3} Y_{4}}^{H^{\prime \prime}}\right|=\left|\mathcal{E}_{Y_{3} Y_{4}}^{H}\right|-1 \text {. By (5.1), } \\
& d_{H^{\prime \prime}}(D)=\left|\mathcal{E}_{X_{3} X_{4}}^{H^{\prime \prime}}\right|+\left|\mathcal{E}_{Y_{3} Y_{4}}^{H^{\prime \prime}}\right|+\left|\mathcal{E}_{O}^{H^{\prime \prime}}\right| \\
& =\left|\mathcal{E}_{X_{3} X_{4}}^{H}\right|+\left|\mathcal{E}_{Y_{3} Y_{4}}^{H}\right|+\left|\mathcal{E}_{O}^{H^{\prime \prime}}\right|-1 \\
& \geq h+1+\left|\mathcal{E}_{O}^{H^{\prime \prime}}\right|-\frac{\left|\mathcal{E}_{X_{3} X_{4} \bar{X}}^{H}\right|}{2}-\frac{\left|\mathcal{E}_{Y_{3} Y_{4} \bar{Y}}^{H}\right|}{2} \\
& \geq h+\left|\mathcal{E}_{O}^{H^{\prime \prime}} \cup\left\{E_{0}\right\}\right|-\frac{\left|\mathcal{E}_{X_{3} X_{4} \bar{X}}^{H}\right|}{2}-\frac{\left|\mathcal{E}_{Y_{3} Y_{4} \bar{Y}}^{H}\right|}{2} \\
& =h+\frac{\left|\mathcal{E}_{O}^{H^{\prime \prime}} \cup\left\{E_{0}\right\}\right|-\left|\mathcal{E}_{X_{3} X_{4} \bar{X}}^{H}\right|}{2}+\frac{\left|\mathcal{E}_{O}^{H^{\prime \prime}} \cup\left\{E_{0}\right\}\right|-\left|\mathcal{E}_{Y_{3} Y_{4} \bar{Y}}^{H}\right|}{2} .
\end{aligned}
$$

Since $\mathcal{E}_{X_{3} X_{4} \bar{X}}^{H}$ and $\mathcal{E}_{Y_{3} Y_{4} \bar{Y}}^{H}$ are subsets of $\mathcal{E}_{O}^{H^{\prime \prime}} \cup\left\{E_{0}\right\}$, if one of them is a proper subset of $\mathcal{E}_{O}^{H^{\prime \prime}} \cup\left\{E_{0}\right\}$, then $d_{H^{\prime \prime}}(D)>h$, contrary to $d_{H^{\prime \prime}}(D) \leq h$. Hence $\mathcal{E}_{X_{3} X_{4} \bar{X}}^{H}=\mathcal{E}_{Y_{3} Y_{4} \bar{Y}}^{H}=\mathcal{E}_{O}^{H^{\prime \prime}} \cup\left\{E_{0}\right\}$. Then $E_{0} \in \mathcal{E}_{X_{3} X_{4} \bar{X}}^{H} \cap \mathcal{E}_{Y_{3} Y_{4} \bar{Y}}^{H}$, which means $E_{0}=\left\{a_{1}, a_{2}, \cdots, a_{r}\right\}$ must be $\left(X_{3}, X_{4}, Y_{3}, Y_{4}\right)$ crossing. Thus the new edge $F_{0}^{\prime}=\left\{b_{1}, a_{2}, \cdots, a_{r}\right\}$ must be in $\mathcal{E}_{O}^{H^{\prime \prime}}$. But $F_{0}^{\prime}$ is not an edge in $H$, whence it is not in $\mathcal{E}_{X_{3} X_{4} \bar{X}}^{H}$ and $\mathcal{E}_{Y_{3} Y_{4} \bar{Y}}^{H}$, contrary to $\mathcal{E}_{X_{3} X_{4} \bar{X}}^{H}=\mathcal{E}_{Y_{3} Y_{4} \bar{Y}}^{H}=\mathcal{E}_{O}^{H^{\prime \prime}} \cup\left\{E_{0}\right\}$. This completes the proof of Claim 2.

By Claim 2, the number of edge-cuts of size $h$ of $H^{\prime \prime}$ is less than that of $H$, contrary to (5.4). Thus a contradiction will always occur if (5.2) holds, and so we must have $h=k$.

\subsection{The Proof of Theorem 5.1.5}

The necessity of Theorem 5.1.5 is straightforward. We only need to prove the sufficiency. The argument to prove the sufficiency of Theorem 5.1.5 is similar to that in the proof of Theorem 5.1.4. Theorem 5.1.5 can now be established by combining the two lemmas below.

Lemma 5.3.1. (Gale [30], Ryser [65], See also Page 5 of Berge [1])

A nonincreasing integer sequence $d=\left(d_{1}, d_{2}, \cdots, d_{n}\right)$ is the degree sequence of an $r$-uniform 
hypergraph (possibly with multiple edges) if and only if

(i) $\sum_{i=1}^{n} d_{i}$ is a multiple of $r$;

(ii) $\sum_{i=1}^{n} d_{i} \geq r d_{1}$.

Lemma 5.3.2. An r-uniform multi-hypergraphic sequence $d=\left(d_{1}, d_{2}, \cdots, d_{n}\right)$ has a $k$-edgeconnected realization if and only if

(i) $d_{i} \geq k$ for $i=1,2, \cdots, n$;

(ii) $\sum_{i=1}^{n} d_{i} \geq \frac{r(n-1)}{r-1}$ if $k=1$.

Proof: The proof is essentially identical to that of Theorem 1.4 (except that now we do not need to avoid multiple edges), thus, it is omitted here.

\subsection{Concluding remark}

A hypergraph $H$ is linear if for any two distinct edges $E$ and $F$ in $H,|E \cap F| \leq 1$. A sequence $d$ is linear hypergraphic if there is a linear hypergraph with degree sequence $d$. Usually problems of linear hypergraphic sequences are more difficult than those of hypergraphic sequences. The proof of Theorem 5.1.4 cannot be applied to linear uniform hypergraphic sequences since the graphs constructed in the proof may not be linear. However, we believe that the following analog of Theorem 5.1.4 for linear $r$-uniform hypergraphs holds.

Conjecture 5.4.1. A linear $r$-uniform hypergraphic sequence $d=\left(d_{1}, d_{2}, \cdots, d_{n}\right)$ has a $k$-edgeconnected realization if and only if

(i) $d_{i} \geq k$ for $i=1,2, \cdots, n$;

(ii) $\sum_{i=1}^{n} d_{i} \geq \frac{r(n-1)}{r-1}$ if $k=1$. 


\section{Chapter 6}

\section{Augmenting and preserving partition connectivity of a hypergraph}

\subsection{The Problem}

The problem of edge connectivity augmentation seems to be initiated by Watanabe and Nakamura [71], in which they investigated the minimum number of edges that must be added to a graph $G$ so that the resulting graph is $k$-edge-connected, for given integer $k$ and graph $G$. Frank [27] provided an efficient algorithm to solve this kind of problems. For connectivity augmentation in graphs and hypergraphs, two recent survey papers [41] and [69] are very informative.

Frank, Király and Kriesell [29] introduced $k$-partition-connected hypergraphs as a generalization of $k$-edge-connected hypergraphs. The augmentation and preservation problems related to partition connectivity of graphs and hypergraphs have been investigated in $[28,37,42,47,48]$, among others.

Theorem 6.1.1. Let $G$ be a graph and $k$ be a positive integer. The following are equivalent.

(1) There exists an edge set $X$ such that $G+X$ is $k$-partition-connected.

(2) (Frank and Király, a weaker statement of Theorem 5.2 of [28]) $|X| \geq k(|P|-1)-e(P)$ for every partition $P$ of $V(G)$, where $e(P)$ is the number of edges whose ends are in different classes of $P$.

(3) (Haas, Theorem 1 of [37]) $|X|=k(|V(G)|-1)-|E(G)|$ and for subgraphs $S$ of $G$ with at least two vertices, $|E(S)| \leq k(|V(S)|-1)$.

Theorem 6.1.2. (Király and Makai, a weaker statement of Corollary 4.13 of [42]) Let $H$ be a hypergraph and $k$ be a positive integer. The following are equivalent.

(1) There exists a hyperedge set $X$ such that $H+X$ is k-partition-connected. 
(2) $|X| \geq k(|P|-1)-e(P)$ for every partition $P$ of $V(H)$, where $e(P)$ is the number of hyperedges intersecting at least two classes of $P$.

Liu, Lai and Chen [48] generalize Theorem 6.1.1 and find the exact minimum number of edges that must be added to make the resulting graph be $k$-partition-connected.

The research in this paper is motivated by the results above. Our goal is to determine the minimum number of hyperedges in a hypergraph whose addition makes the resulting hypergraph $k$-partition-connected (Theorem 6.5.4 and 6.5.8 show the exact minimum value and a minimax formula). We also characterize the hyperedges in a $k$-partition-connected hypergraph whose removal will preserve the $k$-partition-connectedness of the hypergraph (Theorem 6.6.2).

Relevant definitions and preliminaries will be presented in Section 6.2. Undefined terms can be found in [1] for hypergraphs and [6] for graphs. In Section 6.3, uniformly dense hypergraphs and their relationship with partition connectivity of hypergraphs will be discussed. A few useful tools (Theorem 6.4.4 and 6.4.9) will be developed in Section 6.4. These tools will be applied to the studies of the augmentation and preservation problems of partition connectivity of hypergraphs in Sections 6.5 and 6.6.

\subsection{Notations and Preliminaries}

A hypergraph $H$ is a hyperforest if for every nonempty subset $U \subseteq V(H),|\mathcal{E}(H[U]) \leq| U \mid-1$. A hyperforest $T$ is a hypertree if $|\mathcal{E}(T)|=|V(T)|-1$. For a hypergraph $H$, let $\tau(H)$ be the maximum number of edge-disjoint spanning hypertrees in $H$ and $a(H)$ be the minimum number of edge-disjoint hyperforests whose union is $\mathcal{E}(H)$. For a graph $G, \tau(G)$ is the spanning tree packing number of $G$ and $a(G)$ is the arboricity of $G$.

Theorem 1.1.1 shows that the $k$-partition-connectedness of a graph $G$ is equivalent to the property that $G$ has $k$ edge-disjoint spanning trees, while Theorem 1.1.2 characterizing graphs that can be decomposed to at most $k$ forests. Frank, Király and Kriesell [29] extended both results to hypergraphs.

Theorem 6.2.1. (Frank, Király and Kriesell [29]) Let $H$ be a hypergraph and $k$ be a positive integer. Then $\tau(H) \geq k$ if and only if for every $X \subseteq \mathcal{E}(H),|X| \geq k(\omega(H-X)-1$ ) (or equivalently, $H$ is $k$-partition-connected).

By Theorem 6.2.1, $\tau(H)$ is the partition connectivity of $H$ and a hypertree is a minimal partition-connected hypergraph.

Theorem 6.2.2. (Frank, Király and Kriesell [29]) Let $H$ be a hypergraph and $k$ be a positive integer. Then $\alpha(H) \leq k$ if and only if for any subgraph $S,|\mathcal{E}(S)| \leq k(|V(S)|-1)$. 
Let $H_{1}, H_{2}, \cdots, H_{c}$ be the components of a hypergraph $H$ such that each $H_{i}$ has a spanning hypertree $T_{i}$ for $i=1,2, \cdots, c$. Then $\cup_{i} T_{i}$ is a hyperbase of $H$. By definition, if $H$ is connected, then a hyperbase is a spanning hypertree of $H$. Theorem 6.2 .1 implies the following corollary.

Corollary 6.2.3. A hypergraph $H$ has $k$ edge-disjoint hyperbases if and only if for every $X \subseteq$ $\mathcal{E}(H),|X| \geq k(\omega(H-X)-\omega(H))$.

\subsection{Uniformly Dense Hypergraphs}

In this section, we consider only loopless hypergraphs. Let $E$ be a hyperedge in a hypergraph $H$. By $H / E$ we denote the hypergraph obtained from $H$ by contracting the hyperedge $E$ into a new vertex $v_{0}$ and by removing resulting loops if there are any. That is, $V(H / E)=(V(H) \backslash E) \cup\left\{v_{0}\right\}$ and a hyperedge $E^{\prime} \in \mathcal{E}(H / E)$ if and only if either $E^{\prime}=E^{\prime \prime}$ for some $E^{\prime \prime} \in \mathcal{E}(H)$ with $E^{\prime \prime} \cap E=\emptyset$ or $E^{\prime}=\left(E^{\prime \prime} \backslash E\right) \cup\left\{v_{0}\right\}$ for some $E^{\prime \prime} \in \mathcal{E}(H) \backslash\{E\}$ with $E^{\prime \prime} \cap E \neq \emptyset$. The hyperedge $E^{\prime}$ is called the image of $E^{\prime \prime}$ and $E^{\prime \prime}$ is a preimage of $E^{\prime}$. Let $X \subseteq \mathcal{E}(H), H / X$ is a hypergraph obtained from $H$ by contracting all edges in $X$. Let $S$ be a sub-hypergraph of $H, H / S$ denotes $H / \mathcal{E}(S)$.

For any nonempty subset $X \subseteq \mathcal{E}(H)$, the density of $X$ is defined to be

$$
d_{H}(X)=\frac{|X|}{|V(H[X])|-\omega(H[X])} .
$$

We often use $d(H)$ for $d(\mathcal{E}(H))$. If $X \subset \mathcal{E}(H)$, then by the definition of contraction, $d(H / X)=$ $\frac{|\mathcal{E}(H)-X|}{V(H / X)-\omega(H)}$. Following [11], the strength $\eta(H)$ and the fractional arboricity $\gamma(H)$ of a nontrivial hypergraph $H$ are defined, respectively, as

$$
\left.\eta(H)=\min \left\{\frac{|\mathcal{E}(H)-X|}{V(H / X)-\omega(H)}: X \subseteq \mathcal{E}\right]\right\}, \text { and } \gamma(H)=\max \{d(H[X]: X \subseteq \mathcal{E}\},
$$

where the minimum or maximum is taken over all edge subsets of $\mathcal{E}$ so that the denominators are nonzero. By convention, $\eta\left(K_{1}\right)=d\left(K_{1}\right)=\gamma\left(K_{1}\right)=\infty$. It follows immediately that for any loopless nontrivial hypergraph $H$,

$$
\eta(H) \leq d(H) \leq \gamma(H)
$$

Let $H$ be a hypergraph and $t$ be a positive integer. A $t$-packing of $H$ is a family $\mathscr{F}$ of hyperbases in $H$ such that each hyperedge of $H$ is in at most $t$ members of $\mathscr{F}$. Let $\eta_{t}(H)$ denote the largest cardinality of $t$-packings of $H$. Dually, a $t$-covering of $H$ is a family $\mathscr{F}$ of hyperforests in $H$ such that each hyperedge of $H$ is in at least $t$ members of $\mathscr{F}$. Let $\gamma_{t}(H)$ denote the smallest cardinality of $t$-coverings of $H$. (If $H$ has a loop, then $\gamma_{t}(H)=\infty$.)

The proposition below follows from Theorems 6.2.1, 6.2.2 and Corollary 6.2.3. 
Proposition 6.3.1. For any hypergraph $H$, each of the following holds.

(i) $\alpha(H)=\gamma_{1}(H)=\lceil\gamma(H)\rceil$.

(ii) $\eta_{1}(H)=\lfloor\eta(H)\rfloor$.

(iii) If $H$ is connected, then $\tau(H)=\eta_{1}(H)$.

Let $H=(V, \mathcal{E})$ be a hypergraph and let $t>0$ be an integer. The hypergraph $H^{(t)}=\left(V, \mathcal{E}^{\prime}\right)$ has the same vertex set $V$, where $\mathcal{E}^{\prime}$ is obtained by replacing each hyperedge in $\mathcal{E}$ by a set of $t$ parallel hyperedges.

Theorem 6.3.2. Let $H$ be a hypergraph and $s, t>0$ be integers. Each of the following holds.

(i) $H$ has a t-packing of cardinality $s$ if and only if $\eta(H) \geq s / t$.

(ii) $H$ has a t-covering of cardinality $s$ if and only if $\gamma(H) \leq s / t$.

(iii) $\eta_{t}(H)=\lfloor t \eta(H)\rfloor$ and $\gamma_{t}(H)=\lceil t \gamma(H)\rceil$.

Proof: It suffices to prove (i) and (ii).

(i) $H$ has a $t$-packing of cardinality $s$ if and only if $H^{(t)}$ has $s$ edge-disjoint hyperbases. By Proposition 6.3.1, this is equivalent to $\eta\left(H^{(t)}\right) \geq \eta_{1}\left(H^{t}\right) \geq s$. By definition, it is equivalent to $t \eta(H) \geq s$, or $\eta(H) \geq s / t$.

(ii) $H$ has a $t$-covering of cardinality $s$ if and only if $H^{(t)}$ can be decomposed into $s$ hyperforests. By Proposition 6.3.1, this is equivalent to $\gamma\left(H^{(t)}\right) \leq s$. By definition, it is equivalent to $t \gamma(H) \leq$ $s$, or $\gamma(H) \leq s / t$.

A hypergraph $H$ is uniformly dense if $d(H)=\gamma(H)$. The next result extends Theorem 6 of $[11]$.

Theorem 6.3.3. Let $H$ be a hypergraph. The following are equivalent.

(i) $\eta(H)=\gamma(H)$.

(ii) $\eta(H)=d(H)$.

(iii) $d(H)=\gamma(H)$.

(iv) There is a family $\mathscr{F}$ of hyperbases of $H$ and a positive integer $t$ such that $\mathscr{F}$ is both a t-packing and a t-covering.

Proof: (i) $\Rightarrow$ (ii) and (i) $\Rightarrow$ (iii) follow from (6.2).

(ii) $\Rightarrow$ (iv): Suppose that $\eta(H)=d(H)=\frac{h}{t}$ for some integers $h, t>0$. By Theorem 6.3.2 (iii), $h=t \eta(H)=\eta_{t}(H)$, and so $H$ has a family $\mathscr{F}=\left\{T_{1}, T_{2}, \cdots, T_{h}\right\}$ of hyperbases such that every hyperedge $E \in \mathcal{E}(H)$ is in at most $t$ members of $\mathscr{F}$. As $\eta(H)=d(H)$, we have $\operatorname{t\eta }(H)(|V(H)|-\omega(H))=\eta_{t}(H)(V(H)-\omega(H))=\sum_{i=1}^{h}\left|T_{i}\right| \leq t|\mathcal{E}(H)|=t \eta(H)(|V(H)|-\omega(H))$, and so every hyperedge of $H$ is in exactly $t$ members of $\mathscr{F}$. Thus (iv) holds.

(iii) $\Rightarrow\left(\right.$ iv): Let $g \geq t>0$ be integers such that $d(H)=\gamma(H)=\frac{g}{t}$. By Theorem 6.3.2 (iii), $g=t \gamma(H)=\gamma_{t}(H)$, and so $H$ has a family $\mathscr{F}=\left\{B_{1}, B_{2}, \cdots, B_{h}\right\}$ of hyperforests such that every 
hyperedge $E \in \mathcal{E}(H)$ is in at least $t$ members of $\mathscr{F}$. As $\eta(H)=d(H)$, we have $t \gamma(H)(|V(H)|-$ $\omega(H))=\gamma_{t}(H)(V(H)-\omega(H)) \geq \sum_{i=1}^{g}\left|B_{i}\right| \geq t|\mathcal{E}(H)|=t \gamma(H)(|V(H)|-\omega(H))$, and so each $B_{i}$ is a hyperbase of $H$ for $1 \leq i \leq g$; and every hyperedge of $H$ is in exactly $t$ members of $\mathscr{F}$. Thus (iv) holds.

(iv) $\Rightarrow($ i): Since $\mathscr{F}$ is a $t$-packing as well as a $t$-covering of cardinality $s$, by Theorem 6.3.2, $\eta(H) \geq \frac{s}{t} \geq \gamma(H) \geq \eta(H)$. Thus (i) holds.

Lemma 6.3.4. Let $H$ be a nontrivial hypergraph and $l \geq 1$ be a fractional number. Then each of the following holds.

(i) If $X \subseteq \mathcal{E}(H)$, then $\eta(H) \leq \eta(H / X)$.

(ii) If $X \subseteq \mathcal{E}(H)$ and $\eta(H[X])>\eta(H)$, then $\eta(H / X)=\eta(H)$.

(iii) If $d(H) \geq l$, then there exists a nonempty subset $X \subseteq \mathcal{E}(H)$ such that $\eta(H[X]) \geq l$.

Proof: (i) By definition, there exists $Y^{\prime} \subseteq \mathcal{E}(H / X)$ such that $\eta(H / X)=d\left((H / X) / Y^{\prime}\right)$. Let $Y \subseteq \mathcal{E}(H)$ be a preimage of $Y^{\prime}$. Then $\eta(H / X)=d\left((H / X) / Y^{\prime}\right)=d(H /(X \cup Y)) \geq \eta(H)$. (If $H[X]$ is spanning, then $\eta(H / X)=\infty$.)

(ii) It suffices to show that $\eta(H) \geq \eta(H / X)$. By definition, there exists a nonspanning subset $T$ of $\mathcal{E}(H)$ such that $\eta(H)=d(H / T)=\frac{|\mathcal{E}(H) \backslash T|}{|V(H / T)|-\omega(H)}$. We use $X^{c}$ to denote $\mathcal{E}(H) \backslash X$ and let $X \cap T=T_{1}$ and $X^{c} \cap T=T_{2}$. Then

$$
\eta(H)=\frac{\left|X \backslash T_{1}\right|+\left|X^{c} \backslash T_{2}\right|}{|V(H / T)|-\omega(H)} .
$$

If $V\left(H\left[T_{1}\right]\right)=V(H[X])$, then let $T_{2}^{\prime} \subseteq \mathcal{E}\left(H / T_{1}\right)$ be the image of $T_{2}$. By definition, $\eta(H / X)=$ $\eta\left(H / T_{1}\right) \leq d\left(\left(H / T_{1}\right) / T_{2}^{\prime}\right)=d(H / T)=\eta(H)$. Therefore, we assume that $V\left(H\left[T_{1}\right]\right) \neq V(H[X])$.

By definition, $\eta(H[X]) \leq d\left(H[X] / T_{1}\right)=\frac{\left|X \backslash T_{1}\right|}{\left|V\left(H[X] / T_{1}\right)\right|-\omega(H[X])}$, and so

$$
\left|X \backslash T_{1}\right| \geq \eta(H[X])\left(\left|V\left(H[X] / T_{1}\right)\right|-\omega(H[X])\right)>\eta(H)\left(\left|V\left(H[X] / T_{1}\right)\right|-\omega(H[X])\right) .
$$

By (6.3) and (6.4),

$$
\eta(H)\left(|V(H / T)|-\omega(H)-\left|V\left(H[X] / T_{1}\right)\right|+\omega(H[X])\right)>\left|X^{c} \backslash T_{2}\right| .
$$

We also have $\left|V\left(H /\left(X \cup T_{2}\right)\right)\right|=|V(H / T)|-\left|V\left(H[X] / T_{1}\right)\right|+\omega(H[X])$. By (6.5),

$$
\eta(H)\left(\left|V\left(H /\left(X \cup T_{2}\right)\right)\right|-\omega(H)\right)>\left|X^{c} \backslash T_{2}\right| .
$$

Since the inequality (6.6) is strict, $\left|V\left(H /\left(X \cup T_{2}\right)\right)\right|-\omega(H) \neq 0$, and so

$$
\eta(H)>\frac{\left|X^{c} \backslash T_{2}\right|}{\left|V\left(H /\left(X \cup T_{2}\right)\right)\right|-\omega(H)} .
$$


Let $T_{2}^{\prime} \subseteq \mathcal{E}(H / X)$ be the image of $T_{2}$. Since $\left|V\left(H /\left(X \cup T_{2}\right)\right)\right|-\omega(H) \neq 0, V\left(H / X\left[T_{2}^{\prime}\right]\right) \neq$ $V(H / X)$. By definition, $\eta(H / X) \leq d\left((H / X) / T_{2}^{\prime}\right)=d\left(H /\left(X \cup T_{2}\right)\right)$, and thus

$$
\eta(H / X) \leq \frac{\mathcal{E}(H) \backslash\left(X \cup T_{2}\right)}{\left|V\left(H /\left(X \cup T_{2}\right)\right)\right|-\omega(H)} \leq \frac{\left|X^{c} \backslash T_{2}\right|}{\left|V\left(H /\left(X \cup T_{2}\right)\right)\right|-\omega(H)} .
$$

By (6.7) and (6.8), $\eta(H)>\eta(H / X)$, which is impossible by (i). This completes the proof.

(iii) Since $\gamma(H) \geq d(H) \geq l$, by the definition of $\gamma(H)$, there exists a nonempty subset $X \subseteq \mathcal{E}(H)$ such that $\gamma(H)=d(H[X])$. Thus $\gamma(H[X]) \leq \gamma(H)=d(H[X]) \leq \gamma(H[X])$, and we have $\gamma(H[X])=d(H[X]) \geq l$. By Theorem 6.3.3, $\eta(H[X])=d(H[X])=\gamma(H[X]) \geq l$.

Lemma 6.3.5. Let $H$ be a nontrivial hypergraph. The following are equivalent.

(i) $H$ is uniformly dense.

(ii) For any nontrivial sub-hypergraph $S, d(S) \leq \eta(H)$.

(iii) For any nontrivial sub-hypergraph $S, \eta(S) \leq \eta(H)$.

Proof: (i) $\Longrightarrow$ (ii). As $H$ is uniformly dense, $d(S) \leq \gamma(H)=\eta(H)$, and so (ii) holds.

(ii) $\Longrightarrow$ (iii). By $(6.2), \eta(S) \leq d(S) \leq \eta(H)$, and so (iii) holds.

(iii) $\Longrightarrow$ (i). If $H$ is not uniformly dense, then by (6.2) and (6.1), for some subset $X \subseteq \mathcal{E}$, $d(X)=\gamma(H)>\eta(H)$. Let $S=H[X]$. By (6.1) again, $d(S)=\gamma(S)=\gamma(H)$, and so by Theorem 6.3.3, $\eta(S)=d(S)=\gamma(H)>\eta(H)$, contrary to (iii). This completes the proof.

\subsection{Complete Families and Decomposition Theorems}

Throughout this section, unless otherwise stated, sub-hypergraphs of a hypergraph $H$ are all edge induced, and so we adopt the convention to use a subset $S$ of $\mathcal{E}(H)$ to denote both the edge subset as well as the edge induced sub-hypergraph of $H$. In particular, if $S_{1}, S_{2}$ are subhypergraphs of $H$, then $S_{1} \cup S_{2}$ denotes the sub-hypergraph of $H$ induced by the edge subset $S_{1} \cup S_{2}$.

Let $k \geq 1$ be an integer and let $\mathcal{T}_{k}$ be the family of all $k$-partition-connected hypergraphs. Thus $K_{1} \in \mathcal{T}_{k}$ and every hypergraph in $\mathcal{T}_{k}$ is connected. A decomposition theorem that partitions the hyperedges set $\mathcal{E}$ of a hypergraph $H$ according to the different level of partition connectivity, and other related results, will be presented in Theorem 6.4.4, 6.4.9, Proposition 6.4.1 and 6.4.6 in this section. Connected graph families satisfying (C1), (C2) and (C3) as stated in Proposition 6.4.1 are often referred as complete families, as seen in $[9,12,44]$, among others.

Proposition 6.4.1. For any positive integer $k$, each of the following statements holds.

(C1) $\mathcal{T}_{k} \neq \emptyset$.

(C2) If $E \in \mathcal{E}(H)$ and $H \in \mathcal{T}_{k}$, then $H / E \in \mathcal{T}_{k}$.

(C3) If for some $S \subset \mathcal{E}(H)$, both $S, H / S \in \mathcal{T}_{k}$, then $H \in \mathcal{T}_{k}$. 
Proof: Since $K_{1} \in \mathcal{T}_{k},(\mathrm{C} 1)$ holds.

Let $E=\left\{v_{1}, v_{2}, \cdots, v_{|E|}\right\}$ and $v$ be the vertex of $H / E$ onto which $E$ is contracted. Let $\pi=\left\{V_{1}, V_{2}, \cdots, V_{|\pi|}\right\}$ denote a partition of $V(H / E)$. Without loss of generality, we assume that $v \in V_{1}$. Define $V_{1}^{\prime}=\left(V_{1} \backslash\{v\}\right) \cup\left\{v_{1}, v_{2}, \cdots, v_{|E|}\right\}$. Then $\pi^{\prime}=\left\{V_{1}^{\prime}, V_{2}, \cdots, V_{|\pi|}\right\}$ is a partition of $V(E)$. Since $H \in \mathcal{T}_{k}, e\left(\pi^{\prime}\right) \geq k\left(\left|\pi^{\prime}\right|-1\right)=k(|\pi|-1)$. By the definition of contraction, $e(\pi)=e\left(\pi^{\prime}\right) \geq k(|\pi|-1)$, whence $H / E \in \mathcal{T}_{k}$, and so (C2) follows.

Let $\pi=\left\{V_{1}, V_{2}, \cdots, V_{|\pi|}\right\}$ be a partition of $H$. Without lost of generality, we assume that for some integer $t \geq 1, V_{j} \cap V(S) \neq \emptyset$ for $1 \leq j \leq t$, and $V_{j} \cap V(S)=\emptyset$ for $t+1 \leq j \leq|\pi|$. Then $\pi_{1}=\left\{V_{1} \cap V(S), V_{2} \cap V(S), \cdots, V_{t} \cap V(S)\right\}$ is a partition of $V(S)$. As $S \in \mathcal{T}_{k}, e\left(\pi_{1}\right) \geq$ $k\left(\left|\pi_{1}\right|-1\right)=k(t-1)$. Moreover, let $\pi_{2}=\left\{V_{0}, V_{t+1}, V_{t+2}, \cdots, V_{|\pi|}\right\}$ be a partition of $V(H / S)$. As $H / S \in \mathcal{T}_{k}, e\left(\pi_{2}\right) \geq k\left(\left|\pi_{2}\right|-1\right)=k(|\pi|-t)$. It follows that $e(\pi)=e\left(\pi_{1}\right)+e\left(\pi_{2}\right) \geq k(|\pi|-1)$, and so $H \in \mathcal{T}_{k}$. This proves (C3).

Corollary 6.4.2. If $S_{1}$ and $S_{2}$ are sub-hypergraphs of a hypergraph $H$ such that $S_{1}, S_{2} \in \mathcal{T}_{k}$ and $V\left(S_{1}\right) \cap V\left(S_{2}\right) \neq \emptyset$, then $S_{1} \cup S_{2} \in \mathcal{T}_{k}$.

Proof: Let $H=S_{1} \cup S_{2}$. Since $S_{1} \in \mathcal{T}_{k}$, by Proposition 6.4.1(C2), $H / S_{2} \in \mathcal{T}_{k}$. Since $S_{2} \in \mathcal{T}_{k}$, by Proposition 6.4.1(C3), $H \in \mathcal{T}_{k}$.

Let $H$ be a nontrivial partition-connected hypergraph. For any positive integer $r$, a nontrivial sub-hypergraph $S$ of $H$ is $\mathcal{T}_{r}$-maximal or $r$-maximal for short, if $S \in \mathcal{T}_{r}$ and if there is no sub-hypergraph $K$ of $H$ such that $K$ contains $S$ properly and such that $K \in \mathcal{T}_{r}$. A $\mathcal{T}_{r}$-maximal sub-hypergraph $S$ of $H$ is an $r$-region if $r=\tau(S)$. Sometimes an $r$-region is called a region if $r$ is not specified. We define $\bar{\tau}(H)=\max \{r: H$ has a sub-hypergraph as an $r$-region $\}$.

Lemma 6.4.3. Let $S$ be a nontrivial connected sub-hypergraph of $H$ and $r$ be a positive integer. If $\tau(S)=r$, then there is always a region $L$ of $H$ with $S \subseteq \mathcal{E}(L)$ and with $\tau(L) \geq r$.

Proof: If $S$ is $r$-maximal, then $L=S$ is an $r$-region of $H$. Otherwise, $H$ has a connected sub-hypergraph $L$ properly containing $S$ with $\tau(L) \geq r$ and such that $L$ is maximal with respect to these properties. Since $H$ is finite, $L$ exists and so $L$ is a desirable region.

Theorem 6.4.4. Let $H$ be a nontrivial partition-connected hypergraph. Then

(i) There exist a positive integer $m$ and an $m$-tuple $\left(i_{1}, i_{2}, \cdots, i_{m}\right)$ of positive integers with

$$
\tau(H)=i_{1}<i_{2}<\cdots<i_{m}=\bar{\tau}(H)
$$

and a sequence of edge subsets

$$
\mathcal{E}_{m} \subset \cdots \subset \mathcal{E}_{2} \subset \mathcal{E}_{1}=\mathcal{E}(H)
$$


such that each component of the induced sub-hypergraph $H\left[\mathcal{E}_{j}\right]$ is an r-region of $H$ for some $r$ with $r \geq i_{j}$ where $1 \leq j \leq m$, and such that at least one component $S$ in $H\left[\mathcal{E}_{j}\right]$ is an $i_{j}$-region of $H$.

(ii) If $S$ is a sub-hypergraph of $H$ with $\tau(S) \geq i_{j}$, then $\mathcal{E}(S) \subseteq \mathcal{E}_{j}$.

(iii) The integer $m$ and the sequence of edge subsets are uniquely determined by $H$.

Proof: (i) Let $\mathcal{R}(H)$ denote the collection of all regions of $H$. Since $H$ itself is a region of $H, \mathcal{R}(H)$ is not empty. Since $H$ is a finite hypergraph, $|\mathcal{R}(H)|$ is finite. We define $s p(H)=$ $\{\tau(S): S \in \mathcal{R}(H)$ is nontrivial $\}$. Then $|s p(H)|$ is finite and $|s p(H)| \geq 1$. Let $m=|s p(H)|$ and $s p(H)=\left\{i_{1}, i_{2}, \cdots, i_{m}\right\}$ with $i_{1}<i_{2}<\cdots<i_{m}$. Since $H \in \mathcal{R}(H), \tau(H) \geq i_{1}$. If $\tau(H)>i_{1}$, then for some region $S \in \mathcal{R}(H), \tau(S)=i_{1}<\tau(H)$, contrary to the fact that $S$ is a region of $H$. Hence we must have $\tau(H)=i_{1}$.

For each $j \in\{1,2, \cdots, m\}$, we define $\mathcal{E}_{j}=\bigcup_{\tau(S) \geq i_{j}, S \in \mathcal{R}(H)} \mathcal{E}(S)$. As $\mathcal{T}_{i_{1}} \supset \mathcal{T}_{i_{2}} \supset \cdots \supset \mathcal{T}_{i_{m}}$, we have $\mathcal{E}_{1} \supset \mathcal{E}_{2} \supset \cdots \supset \mathcal{E}_{m}$. In particular, $\mathcal{E}_{1}=\cup_{\tau(S) \geq i_{1}} \mathcal{E}(S)=\cup_{\tau(S) \geq \tau(H)} \mathcal{E}(S)=\mathcal{E}(H)$.

Claim 1. For any $j \in\{1,2, \cdots, m\}$, each component of $H\left[\mathcal{E}_{j}\right]$ is an $r$-region of $H$ with $r \geq i_{j}$. Proof of Claim 1. Let $L$ be a nontrivial component of $H\left[\mathcal{E}_{j}\right]$. By the definition of $\mathcal{E}_{j}$, we may assume that there are $s$ regions $L_{1}, L_{2}, \cdots, L_{s}$ such that each $L_{t}$ is an $r_{t}$-region with $r_{t} \geq i_{j}$ for $1 \leq t \leq s$, and such that $L=\cup_{t=1}^{s} L_{t}$. Without loss of generality, we may assume that $r_{1} \leq r_{2} \leq \cdots \leq r_{s}$. If $s \geq 2$, then $L_{1}$ must share a common vertex with some $L_{t}$ with $t \geq 2$ since $L$ is connected. By Corollary 6.4.2, $L_{1} \cup L_{t} \in \mathcal{T}_{r_{1}}$, contrary to the fact that $L_{1}$ is $r_{1}$-maximal. Hence $s=1$ and $L=L_{1}$. Thus $L$ is an $r_{1}$-region of $H$ with $r_{1} \geq i_{j}$, completing the proof of the claim.

We still need to show that $H\left[\mathcal{E}_{j}\right]$ contains a component as an $i_{j}$-region of $H$. Since $i_{j} \in s p(H)$, there is an $i_{j}$-region $S$ of $H$, and so $S \subseteq \mathcal{E}_{j}$. The maximality of a region implies that $S$ is a component of $H\left[\mathcal{E}_{j}\right]$.

(ii) follows from Lemma 6.4 .3 and the definition of $\mathcal{E}_{j}$.

(iii) follows from the fact that $\mathcal{R}(H)$ is uniquely determined by $H$.

Theorem 6.4.4 will be a useful tool to prove our main results in the last two sections. It also has a fractional version to be developed in Theorem 6.4.9 below.

Lemma 6.4.5. Let $H$ be a nontrivial connected hypergraph. Then

(i) For some $S \subseteq \mathcal{E}(H), S$ is uniformly dense with $\eta(S)=\gamma(H)$.

(ii) $\bar{\tau}(H)=\lfloor\gamma(H)\rfloor$.

Proof: (i) By (6.1) and (6.2), for some $S \subseteq \mathcal{E}(H), S$ is connected and $d(S)=\gamma(H)$. Hence $d(S) \leq \gamma(S) \leq \gamma(S)=d(S)$, and so by Theorem 6.3.3, $S$ is uniformly dense with $\eta(S)=d(S)=$ $\gamma(H)$. This proves (i). 
(ii) By the definition of $\bar{\tau}(H)$, for some region $R$ of $H, \tau(R)=\bar{\tau}(H)$. By (6.1) and (6.2),

$$
\bar{\tau}(H)=\tau(R) \leq \eta(R) \leq d(R) \leq \gamma(R) \leq \gamma(H)
$$

Let $k>0$ be an integer with $\gamma(H) \geq k$. By (i), for some $S \subseteq \mathcal{E}(H), S$ is connected and $\eta(S)=\gamma(H) \geq k$. By Lemma 6.4.3, $H$ has a region $L$ such that $\tau(L) \geq \tau(S) \geq k$. It follows that $\bar{\tau}(H) \geq \tau(L) \geq k$, and so (ii) must hold.

For each rational number $l \geq 0$, we define $\mathcal{S}_{l}=\{H: \eta(H) \geq l\}$.

Proposition 6.4.6. The hypergraph family $\mathcal{S}_{l}$ has the following properties.

(C1) $\mathcal{S}_{l}$ is nonempty.

(C2) If $H \in \mathcal{S}_{l}$ and $E \in \mathcal{E}(H)$, then $H / E \in \mathcal{S}_{l}$.

(C3) Let $X \subseteq \mathcal{E}(H)$. If $H / X \in \mathcal{S}_{l}$ and $H[X] \in \mathcal{S}_{l}$, then $H \in \mathcal{S}_{l}$.

Proof: As (C1) and (C2) follow from the fact $K_{1} \in \mathcal{S}_{l}$ and Lemma 6.3.4(i), respectively, it suffices to show (C3). Suppose that under the assumption of (C3), we still have $\eta(H)<l$. Then $\eta(H[X]) \geq l>\eta(H)$. By Lemma 6.3.4(ii), $\eta(H / X)=\eta(H)<l$, contrary to $H / X \in \mathcal{S}_{l}$. Thus $H \in \mathcal{S}_{l}$.

Lemma 6.4.7. Let $X$ and $X^{\prime}$ be subsets of $\mathcal{E}(H)$ and $l$ be a rational number. If $\eta(X) \geq l$ and $\eta\left(X^{\prime}\right) \geq l$, then $\eta\left(X \cup X^{\prime}\right) \geq l$.

Proof: By Proposition 6.4.6 (C2), $\left(X \cup X^{\prime}\right) / X=X^{\prime} /\left(X \cap X^{\prime}\right) \in \mathcal{S}_{l}$. As $X^{\prime} \in \mathcal{S}_{l}$, it follows from Proposition 6.4.6(C3), that $\eta\left(X \cup X^{\prime}\right) \geq l$.

Let $H$ be a nontrivial hypergraph. A subset $S \in \mathcal{E}(H)$ is $\eta$-maximal if for any subset $S^{\prime} \in \mathcal{E}(H)$ with $S \subset S^{\prime}$ properly, we always have $\eta\left(S^{\prime}\right)<\eta(S)$.

Lemma 6.4.8. Let $S$ be a sub-hypergraph of $H$. Then $H$ has an $\eta$-maximal sub-hypergraph $L$ such that $\mathcal{E}(S) \subseteq \mathcal{E}(L)$ and such that $\eta(S) \leq \eta(L)$.

Proof: Let $l=\eta(S)$ and $\mathcal{F}$ be the collection of all sub-hypergraphs $S^{\prime}$ of $H$ with $\eta\left(S^{\prime}\right) \geq l$. Let $X=\cup_{S^{\prime} \in \mathcal{F}} \mathcal{E}\left(S^{\prime}\right)$ and $L=H[X]$. By Lemma 6.4.7, $\eta(L) \geq l$. By the definition of $L, L$ is $\eta$-maximal with $\mathcal{E}(S) \subseteq \mathcal{E}(L)$ and $\eta(S) \leq \eta(L)$.

Theorem 6.4.9. Let $H$ be a nontrivial hypergraph. Then each of the following holds.

(i) There exist a positive integer $m$ and an $m$-tuple $\left(l_{1}, l_{2}, \cdots, l_{m}\right)$ of positive rational numbers with

$$
\eta(H)=l_{1}<l_{2}<\cdots<l_{m}=\gamma(H)
$$


and a sequence of edge subsets

$$
J_{m} \subset \cdots \subset J_{2} \subset J_{1}=\mathcal{E}(H)
$$

such that for each $i$ with $1 \leq i \leq m, J_{i}$ is $\eta$-maximal with $\eta\left(H\left[J_{i}\right]\right)=l_{i}$.

(ii) The integer $m$ and the sequences above are uniquely determined by $H$.

Proof: Let $\mathcal{R}(H)$ denote the collection of all $\eta$-maximal sub-hypergraphs of $H$. Then $H \in \mathcal{R}(H)$ and $|\mathcal{R}(H)|$ are finite. Let $s p_{\eta}(H)=\{\eta(S): S \in \mathcal{R}(H)\}, m=\left|s p_{\eta}(H)\right|$ and $s p_{\eta}(H)=$ $\left\{l_{1}, l_{2}, \cdots, l_{m}\right\}$ such that $l_{1}<l_{2}<\cdots<l_{m}$.

Since $H \in \mathcal{R}(H), \eta(H) \geq l_{1}$. If for some $K \in \mathcal{R}(H)$, with $\eta(K)=l_{1}<\eta(H)$, then $K$ is not $\eta$-maximal. Therefore, $\eta(H)=l_{1}$. By Lemma 6.4.5(i), $\gamma(H) \leq l_{m}$. If for some $K \in \mathcal{R}(H)$, with $\eta(K)=l_{m}>\gamma(H)$, then by (6.2), $d(K) \geq \eta(K)>\gamma(H)$, contrary to (6.1). Therefore, $\gamma(H)=l_{m}$.

Fix an $i$ with $1 \leq i \leq m$, by the definition of $l_{i}$, for some $S \in \mathcal{R}(H), \eta(S)=l_{i}$. Define $J_{i}$ to be the set of all hyperedges of $H$ which are in some $S \in \mathcal{R}(H)$ with $\eta(S)=l_{i}$. Then by Proposition 6.4.6 (C3), $J_{m} \subset \cdots \subset J_{2} \subset J_{1}=\mathcal{E}(H)$. This proves (i).

(ii) follows from the fact that $\mathcal{R}(H)$ is uniquely determined by $H$.

The $m$-tuple $\left(l_{1}, l_{2}, \cdots, l_{m}\right)$ in (6.9) and the sequence $J_{1}, J_{2}, \cdots, J_{m}$ in (6.10) are referred as the $\eta$-spectrum and the $\eta$-decomposition of $H$, respectively.

Corollary 6.4.10. Let $H$ be a nontrivial hypergraph with $\eta$-spectrum and $\eta$-decomposition described in Theorem 6.4 .9 with $m>1$. Then $H / J_{2}$ is uniformly dense with $\eta\left(H / J_{2}\right)=\gamma\left(H / J_{2}\right)=$ $\eta(H)$.

Proof: Since $m>1, \eta\left(H\left[J_{2}\right]\right)=l_{2}>l_{1}=\eta(H)$. By Lemma 6.3.4(ii), $\eta\left(H / J_{2}\right)=\eta(H)=l_{1}$. It remains to show that $\gamma\left(H / J_{2}\right)=\eta\left(H / J_{2}\right)$.

If not, then by Lemma 6.4.5(i) and by (6.1) and (6.2), for some $J^{\prime} \subset \mathcal{E}\left(H / J_{2}\right), \eta\left(H / J_{2}\left[J^{\prime}\right]\right)=$ $d_{H / J_{2}}\left(J^{\prime}\right)=\gamma\left(H / J_{2}\right)>\eta\left(H / J_{2}\right)=l_{1}$. Let $J^{\prime \prime} \subseteq \mathcal{E}(H)$ be a preimage of $J^{\prime}$. Then $J^{\prime \prime} \cap J_{2}=\emptyset$ and, since $J_{2}$ is $\eta$-maximal, $\eta\left(J^{\prime \prime} \cup J_{2}\right)<\eta\left(J_{2}\right)=l_{2}$. By Lemma 6.3.4(ii), $\eta\left(J^{\prime \prime} \cup J_{2}\right)=$ $\eta\left(\left(J^{\prime \prime} \cup J_{2}\right) / J_{2}\right)=\eta\left(H / J_{2}\left[J^{\prime}\right]\right)>l_{1}$. By Lemma 6.4.8, $H$ has an $\eta$-maximal sub-hypergraph $L$ with $\eta(L) \geq \eta\left(J^{\prime \prime} \cup J_{2}\right)$ with $J^{\prime \prime} \cup J_{2} \subseteq L$. If $\eta(L) \geq l_{2}$, then $L \subseteq J_{2}$, contrary to $J^{\prime \prime} \cap J_{2}=\emptyset$. Hence $l_{2}>\eta(L) \geq \eta\left(J^{\prime \prime} \cup J_{2}\right)>l_{1}$, and so the $\eta$-spectrum of $H$ should include $\eta(L)$, contrary to the uniqueness of the $\eta$-spectrum of $H$.

Corollary 6.4.11. Let $H$ be a hypergraph with $\eta$-spectrum (6.9). Then $H$ is uniformly dense if and only if $m=1$. 


\subsection{Augmenting Partition Connectivity of a Hypergraph}

Throughout this section, $k>0$ denotes an integer, and $H$ denotes a hypergraph. If $X$ is a collection of (not necessarily distinct) subsets of $V(H)$ and $X \cap \mathcal{E}(H)=\emptyset$, then we use $H+X$ to denote the hypergraph $(V(H), \mathcal{E} \cup X)$. Define $f(H, k)$ to be the minimum number of hyperedges that must be added to $H$ so that the resulting hypergraph is $k$-partition-connected. By Theorem 6.2.1, it suffices to investigate the minimum number of hyperedges that must be added to $H$ so that the resulting hypergraph has $k$ edge-disjoint spanning hypertrees. In this section, we determine the value of $f(H, k)$ together with a min-max formula (Theorem 6.5.4 and 6.5.8). Matroid arguments will be used in some of the proofs, and we refer to [62] for undefined terms for matroid theory.

Lemma 6.5.1. Every hyperforest in a partition-connected hypergraph is a spanning sub-hypergraph of a hypertree.

Proof: Lorea [51] proved that all hyperforests of a hypergraph $H$ form the family of independent sets of a matroid $M_{H}$, called the circuit matroid of $H$, on $\mathcal{E}(H)$. Frank, Király and Kriesell [29] proved that, if $H$ is partition-connected, then any spanning hypertree of $H$ is a base of $M_{H}$. It follows that any hyperforest in a partition-connected hypergraph can be augmented to a hypertree.

Lemma 6.5.2. Suppose that $\tau(H)<k$. If $\gamma(H) \leq k$, then there exists an edge set $X$ with $|X|=k(|V(H)|-1)-|\mathcal{E}(H)|$ such that $H+X$ is the union of $k$ edge-disjoint spanning hypertrees.

Proof: Since $\gamma(H) \leq k$, by Theorem 6.2.2 or Proposition 6.3.1, there exist edge-disjoint spanning hyperforests $F_{1}, F_{2}, \cdots, F_{k}$ such that $\mathcal{E}(H)=\cup_{i=1}^{k} \mathcal{E}\left(F_{i}\right)$. By Lemma 6.5.1, for each $i$ with $1 \leq i \leq k$, each $F_{i}$ can be augmented to a hypertree by adding a set $X_{i}^{\prime}$ of $\left|V\left(F_{i}\right)\right|-1-\left|\mathcal{E}\left(F_{i}\right)\right|$ hyperedges. For each $i$ with $1 \leq i \leq k$, let $X_{i}$ be a set of new hyperedges duplicating the edges in $X_{i}^{\prime}$, and let $X=\cup_{i=1}^{k} X_{i}$. Then $H+X$ is the union of $k$ edge-disjoint spanning hypertrees and $|X|=\sum_{i=1}^{k}\left(\left|V\left(F_{i}\right)\right|-1-\left|\mathcal{E}\left(F_{i}\right)\right|\right)=k(|V(H)|-1)-|\mathcal{E}(H)|$.

Lemma 6.5.3. Let $H$ be a hypergraph and let $W \subseteq \mathcal{E}(H)$ such that every component of $W$ is in $\mathcal{T}_{k}$. If for a set $X^{\prime}$ of hyperedges not in $\mathcal{E}(H / W), H / W+X^{\prime} \in \mathcal{T}_{k}$, then for some set $X$ of hyperedges not in $\mathcal{E}(H), H+X \in \mathcal{T}_{k}$ and $|X|=\left|X^{\prime}\right|$.

Proof: Suppose that $H[W]$ has $c$ components $H_{1}, H_{2}, \cdots, H_{c}$ and let $v_{1}, v_{2}, \cdots, v_{c}$ be the vertices in $H / W$ onto which $H_{1}, H_{2}, \cdots, H_{c}$ are contracted, respectively. We will construct an edge set $X$ from $X^{\prime}$ as follows: Label $X^{\prime}=\left\{E_{1}^{\prime}, E_{2}^{\prime}, \cdots, E_{s}^{\prime}\right\}$, where $s=\left|X^{\prime}\right|$. For each $i$ with $1 \leq i \leq s$, we have the following.

(a) If $E_{i}^{\prime} \cap\left\{v_{1}, v_{2}, \cdots, v_{c}\right\}=\emptyset$, then $E_{i}=E_{i}^{\prime} \in X$. 
(b) If $E_{i}^{\prime} \cap\left\{v_{1}, v_{2}, \cdots, v_{c}\right\}=\left\{v_{i_{1}}, v_{i_{2}}, \cdots, v_{i_{t}}\right\}$ for some $1 \leq t \leq c$, then choose $u_{j} \in V\left(H_{i_{j}}\right)$ for each $j$ with $1 \leq j \leq t$, and define $E_{i}=\left(E_{i}^{\prime} \backslash\left\{v_{1}, v_{2}, \cdots, v_{t}\right\}\right) \cup\left\{u_{1}, u_{2}, \cdots, u_{t}\right\}$.

Therefore, $|X|=\left|X^{\prime}\right|$. By the definition of contraction, $H / W+X^{\prime} \cong(H+X) / W$. Since $H_{i} \in \mathcal{T}_{k}$, and since $(H+X) / W \cong H / W+X^{\prime} \in \mathcal{T}_{k}$, by Proposition 6.4.1, $H+X \in \mathcal{T}_{k}$.

Let $H$ be a partition-connected hypergraph and $i_{j}, \mathcal{E}_{j}$ be defined in Theorem 6.4 .4 for $j=$ $1,2, \cdots, m$. Let $k$ be a positive integer. If $k \leq i_{m}$, we define $i(k)=\min \left\{i_{j}: i_{j} \geq k\right\}$. If $k>i_{m}$, we define $i(k)=\infty$ and $\mathcal{E}_{\infty}=\emptyset$. Let $c_{k}(H)$ be the number of components of $H\left[\mathcal{E}_{i(k)}\right]$ and $w_{k}(H)=\left|V\left(H\left[\mathcal{E}_{i(k)}\right]\right)\right|$. Note that $c_{k}(H)=w_{k}(H)=0$ if $i(k)=\infty$.

Theorem 6.5.4. Let $H$ be a partition-connected hypergraph with $\tau(H)<k$. Then $f(H, k)=$ $k\left(|V(H)|-w_{k}(H)+c_{k}(H)-1\right)-\left(|\mathcal{E}(H)|-\left|\mathcal{E}_{i(k)}\right|\right)$.

Proof: If $\gamma(H)<k$, then by Lemma 6.4.5, $i_{m}=\bar{\tau}(H) \leq \gamma(H)<k$. Then $i(k)=\infty$, and we have $c_{k}(H)=w_{k}(H)=0$. Then the theorem follows from Lemma 6.5.2. Hence we may assume that $\gamma(H) \geq k$.

Let $H^{\prime}=H / \mathcal{E}_{i(k)}$. Then $\left|\mathcal{E}\left(H^{\prime}\right)\right|=|\mathcal{E}(H)|-\left|\mathcal{E}_{i(k)}\right|$ and $\left|V\left(H^{\prime}\right)\right|=|V(H)|-w_{k}(H)+c_{k}(H)$.

Claim 2. $\gamma\left(H^{\prime}\right) \leq k$.

Proof of Claim 2. By contradiction, we assume that $\gamma\left(H^{\prime}\right)>k$.

By Lemma 6.4.5, $H^{\prime}$ has an $r$-region $L^{\prime}$ with $r \geq k$. Suppose that $H\left[\mathcal{E}_{i(k)}\right]$ has $c$ components $H_{1}, H_{2}, \cdots, H_{c}$ and let $v_{1}, v_{2}, \cdots, v_{c}$ be the vertices in $H / \mathcal{E}_{i(k)}$ onto which the components $H_{1}, H_{2}, \cdots, H_{c}$ are contracted, respectively. By Theorem 6.4.4, $\tau\left(H_{i}\right) \geq k$ for $i=$ $1,2, \cdots, c$. If $V\left(L^{\prime}\right) \cap\left\{v_{1}, v_{2}, \cdots, v_{c}\right\}=\emptyset$, then $L^{\prime}$ is a sub-hypergraph of $H$ with $\tau\left(L^{\prime}\right) \geq$ $k$. By Theorem 6.4.4, $\mathcal{E}\left(L^{\prime}\right) \subseteq \mathcal{E}_{i(k)}$, contrary to the fact that $L^{\prime}$ is a sub-hypergraph of $H / \mathcal{E}_{i(k)}$. If $V\left(L^{\prime}\right) \cap\left\{v_{1}, v_{2}, \cdots, v_{c}\right\} \neq \emptyset$, then without loss of generality, we may assume that $V\left(L^{\prime}\right) \cap\left\{v_{1}, v_{2}, \cdots, v_{c}\right\}=\left\{v_{1}, v_{2}, \cdots, v_{t}\right\}$ for some $t \leq c$. Let $\mathcal{E}_{\text {pre }}$ be a preimage of $\mathcal{E}\left(L^{\prime}\right)$ and $L=H\left[\cup_{i=1}^{t} \mathcal{E}\left(H_{i}\right) \cup \mathcal{E}_{\text {pre }}\right]$. Note that $L^{\prime}=L / \cup_{i=1}^{t} \mathcal{E}\left(H_{i}\right)$. Since $L^{\prime} \in \mathcal{T}_{k}$ and each component of $H\left[\cup_{i=1}^{t} \mathcal{E}\left(H_{i}\right)\right]$ is in $\mathcal{T}_{k}$, by Proposition 6.4.1, $L \in \mathcal{T}_{k}$. By Theorem 6.4.4, $\mathcal{E}(L) \subseteq \mathcal{E}_{i(k)}$, contrary to the fact that $L^{\prime}$ is a sub-hypergraph of $H^{\prime}$. This proves the claim.

By Claim 2 and Lemma 6.5.2, there exists an edge set $X^{\prime}$ disjoint from $\mathcal{E}(H)$ with $\left|X^{\prime}\right|=$ $k\left(\left|V\left(H^{\prime}\right)\right|-1\right)-\left|\mathcal{E}\left(H^{\prime}\right)\right|$ such that $H^{\prime}+X^{\prime}$ is the union of $k$ edge-disjoint spanning hypertrees. This is the minimum number of hyperedges that must be added to $H^{\prime}$ in order to have $k$ edgedisjoint spanning hypertrees.

By Lemma 6.5.3 with $W=\mathcal{E}_{i(k)}$, for some edge subset $X$ disjoint from $\mathcal{E}(H)$, with $|X|=\left|X^{\prime}\right|$, such that $H+X \in \mathcal{T}_{k}$. Thus $f(H, k)=k\left(\left|V\left(H^{\prime}\right)\right|-1\right)-\left|\mathcal{E}\left(H^{\prime}\right)\right|=k\left(|V(H)|-w_{k}(H)+c_{k}(H)-\right.$ $1)-\left(|\mathcal{E}(H)|-\left|\mathcal{E}_{i(k)}\right|\right)$. 
In the rest of this section, we present a related min-max formula for $f(H, k)$ (Theorem 6.5.8). For any subset $X \subseteq \mathcal{E}(H)$, define

$$
f_{k}(H, X)=k(\omega(H-X)-1)-|X| \text { and } F_{k}(H)=\max _{X \subseteq \mathcal{E}(H)}\left\{f_{k}(H, X)\right\} .
$$

Note that $F_{k}(H) \geq f_{k}(H, \emptyset)=0$.

Lemma 6.5.5. Let $X \subseteq \mathcal{E}(H)$ be a subset with $f_{k}(H, X)=F_{k}(H)$ and $C$ be a component of $H-X$.

(i) For any subset $X_{C}$ of $\mathcal{E}(C), f_{k}\left(H, X \cup X_{C}\right)=f_{k}(H, X)+f_{k}\left(C, X_{C}\right)$.

(ii) $F_{k}(C)=0$.

(iii) $\tau(C) \geq k$ (and so $C \in \mathcal{T}_{k}$ ).

Proof: (i) $f_{k}\left(H, X \cup X_{C}\right)=k\left(\omega\left(H-\left(X \cup X_{C}\right)\right)-1\right)-\left|X \cup X_{C}\right|=k\left(\omega(H-X)-1+\omega\left(C-X_{C}\right)-\right.$ 1) $-|X|-\left|X_{C}\right|=k(\omega(H-X)-1)-|X|+k\left(\omega\left(C-X_{C}\right)-1\right)-\left|X_{C}\right|=f_{k}(H, X)+f_{k}\left(C, X_{C}\right)$. (ii) By (i), for any $X_{C} \subseteq \mathcal{E}(C), f_{k}\left(C, X_{C}\right)=f_{k}\left(H, X \cup X_{C}\right)-f_{k}(H, X)=f_{k}\left(H, X \cup X_{C}\right)-$ $F_{k}(H) \leq 0$. Thus $F_{k}(C)=0$.

(iii) By (ii), for any $X_{C} \subseteq \mathcal{E}(C), f_{k}\left(C, X_{C}\right) \leq 0$. In particular, for any $X_{C} \subseteq \mathcal{E}(C)$ with $\omega\left(C-X_{C}\right)>1, k\left(\omega\left(C-X_{C}\right)-1\right)-\left|X_{C}\right| \leq 0$. Thus $\frac{\left|X_{C}\right|}{\omega\left(C-X_{C}\right)-1} \geq k$. By Theorem 6.2.1, $\tau(C) \geq k$.

Lemma 6.5.6. If $H$ is connected and $F_{k}(H)=f_{k}(H, \mathcal{E}(H))$, then $\gamma(H) \leq k$.

Proof: Let $S$ be an induced sub-hypergraph of $H$. By the definition of $\gamma(H)$, it suffices to show that $|\mathcal{E}(S)| \leq k(|V(S)|-\omega(S))$. By definition, $F_{k}(H)=f_{k}(H, \mathcal{E}(H))=k(|V(H)|-1)-|\mathcal{E}(H)|$. Let $X=\mathcal{E}(H) \backslash \mathcal{E}(S)$. Then the components of $H-X$ is the components of $S$ and $|V(H)|-|V(S)|$ isolated vertices. Thus $f_{k}(H, X)=k(\omega(H-X)-1)-|X|=k(\omega(S)+|V(H)|-|V(S)|-1)-$ $(|\mathcal{E}(H)|-|\mathcal{E}(S)|)=k(|V(H)|-1)-|\mathcal{E}(H)|+k(\omega(S)-|V(S)|)+|\mathcal{E}(S)|=F_{k}(H)-k(|V(S)|-$ $\omega(S))+|\mathcal{E}(S)|$. Since $f_{k}(H, X) \leq F_{k}(H)$, we have $F_{k}(H)-k(|V(S)|-\omega(S))+|\mathcal{E}(S)| \leq F_{k}(H)$, that is, $|\mathcal{E}(S)| \leq k(|V(S)|-\omega(S))$, completing the proof.

Lemma 6.5.7. Let $H$ be a hypergraph and $X$ be a subset of $\mathcal{E}(H)$ such that $f_{k}(H, X)=F_{k}(H)$. Let $H_{0}=H /(\mathcal{E}(H) \backslash X)$ and $X_{0} \subseteq \mathcal{E}\left(H_{0}\right)$ be the image of $X$. Then $f_{k}\left(H_{0}, X_{0}\right)=F_{k}\left(H_{0}\right)=$ $F_{k}(H)$.

Proof: First noticing that $\omega(H-X)=\omega\left(H_{0}-X_{0}\right)$ and $\left|X_{0}\right| \leq|X|$ (this is because the images of some hyperedges might be loops and will be removed), by the definition of $f_{k}(H, X)$, we have $f_{k}(H, X) \leq f_{k}\left(H_{0}, X\right)$. Thus $F_{k}\left(H_{0}\right) \geq f_{k}\left(H_{0}, X_{0}\right) \geq f_{k}(H, X)=F_{k}(H)$. On the other hand, we may choose $X_{0}^{\prime} \subseteq X_{0}$ such that $F_{k}\left(H_{0}\right)=f_{k}\left(H_{0}, X_{0}^{\prime}\right)$. Let $X^{\prime} \subseteq \mathcal{E}(H)$ be a set of preimages of hyperedges of $X_{0}^{\prime}$. Then $\left|X^{\prime}\right|=\left|X_{0}^{\prime}\right|$. Since $\omega\left(H-X^{\prime}\right)=\omega\left(H_{0}-X_{0}^{\prime}\right)$, we have $f_{k}\left(H, X^{\prime}\right)=f_{k}\left(H_{0}, X_{0}^{\prime}\right)$, and thus $F_{k}(H) \geq f_{k}\left(H, X^{\prime}\right)=f_{k}\left(H_{0}, X_{0}^{\prime}\right)=F_{k}\left(H_{0}\right)$. It follows that $f_{k}\left(H_{0}, X_{0}\right)=F_{k}\left(H_{0}\right)=F_{k}(H)$. 
Theorem 6.5.8. Let $H$ be a connected hypergraph. Then $f(H, k)=F_{k}(H)$.

Proof: Let $X$ be a subset of $\mathcal{E}(H)$ such that $f_{k}(H, X)=F_{k}(H)$. Let $H_{0}=H /(\mathcal{E}(H) \backslash X)$ and $X_{0} \subseteq \mathcal{E}\left(H_{0}\right)$ be the image of $X$. By Lemma 6.5.7, $f_{k}\left(H_{0}, X\right)=F_{k}\left(H_{0}\right)=F_{k}(H)$. By Lemma 6.5.6, $\gamma\left(H_{0}\right) \leq k$. Thus, by Lemma 6.5.2, $f\left(H_{0}, k\right)=k\left(\left|V\left(H_{0}\right)\right|-1\right)-\left|\mathcal{E}\left(H_{0}\right)\right|=$ $f_{k}\left(H_{0}, X_{0}\right)=F_{k}\left(H_{0}\right)=F_{k}(H)$.

Let $W=\mathcal{E}(H) \backslash X$. By Lemma 6.5.5, each component of $W$ is in $\mathcal{T}_{k}$. Let $Y_{0}$ be the edge set with $\left|Y_{0}\right|=f\left(H_{0}, k\right)$ such that $\tau\left(H_{0}+Y_{0}\right) \geq k$. By Lemma 6.5.3, there exists a set $Y$ of hyperedges not in $\mathcal{E}(H)$ such that $H+Y \in \mathcal{T}_{k}$ with $|Y|=\left|Y_{0}\right|$. Thus $f(H, k) \leq f\left(H_{0}, k\right)=$ $F_{k}(H)$.

To prove $f(H, k) \geq F_{k}(H)$, we assume that $Z$ is a set of hyperedges such that $\tau(H+Z) \geq$ $k$ and $|Z|=f(H, k)$. Let $Z^{\prime} \subseteq \mathcal{E}((H+Z) / W)$ be the image of $Z$. Then $\left|Z^{\prime}\right| \leq|Z|$ and $(H+Z) / W=H / W+Z^{\prime}=H_{0}+Z^{\prime}$. Since $\tau(H+Z) \geq k$, by Proposition 6.4.1, $\tau\left(H_{0}+Z^{\prime}\right) \geq k$. Thus $F_{k}(H)=f\left(H_{0}, k\right) \leq\left|Z^{\prime}\right| \leq|Z|=f(H, k)$, completing the proof.

\subsection{Preserving Partition Connectivity of a Hypergraph}

For a positive integer $k$ and a hypergraph $H$ with $\tau(H) \geq k$, we define $\mathcal{E}_{k}(H)=\{E \in \mathcal{E}(H)$ : $\tau(H-E) \geq k\}$. In this section, we determine the set $\mathcal{E}_{k}(H)$ for a $k$-partition-connected hypergraph $H$. Theorem 6.6.2 is the main result.

Lemma 6.6.1. Let $H$ be a hypergraph. if there exists $X \subseteq \mathcal{E}(H)$ such that

(a) $\tau(H / X) \geq k$ and $\tau(H[X]) \geq k$, and

(b) $\mathcal{E}_{k}(H[X])=\mathcal{E}(H[X])$ and $\mathcal{E}_{k}(H / X)=\mathcal{E}(H / X)$, then $\mathcal{E}_{k}(H)=\mathcal{E}(H)$.

Proof: For any $E \in \mathcal{E}(H)$, if $E \in X$, then by $\mathcal{E}_{k}(H[X])=\mathcal{E}(H[X])$, we have $\tau(H[X]-E) \geq k$. We also have $\tau((H-E) /(X-E))=\tau(H / X) \geq k$. By Proposition 6.4.1(C3), $\tau(H-E) \geq k$. If $E \notin X$, then let $E^{\prime} \in \mathcal{E}(H / X)$ be the image of $E$. Since $\mathcal{E}_{k}(H / X)=\mathcal{E}(H / X), \tau\left(H / X-E^{\prime}\right) \geq k$. Thus $\tau((H-E) / X)=\tau\left(H / X-E^{\prime}\right) \geq k$. We also have $\tau((H-E)[X])=\tau(H[X]) \geq k$. By Proposition 6.4.1(C3), $\tau(H-E) \geq k$. Hence $\mathcal{E}_{k}(H)=\mathcal{E}(H)$.

Theorem 6.6.2. Let $k$ be a positive integer and $H$ be a hypergraph with $\tau(H) \geq k$ and $\eta$ decomposition (6.10). Then each of the following holds.

(i) $\mathcal{E}_{k}(H)=\emptyset$ if and only if $d(H)=k$.

(ii) $\mathcal{E}_{k}(H)=\mathcal{E}(H)$ if and only if $\eta(H)>k$.

(iii) If $\eta(H)=k$, then $\mathcal{E}_{k}(H)=J_{2}$.

Proof: (i). Since $\tau(H) \geq k, d(H)=k$ if and only if $|\mathcal{E}(H)|=k(|V(H)|-1$ ), if and only if $H$ is a union of $k$ edge-disjoint spanning hypertrees, and if and only if $\mathcal{E}_{k}(H)=\emptyset$. 
(ii). By Proposition 6.3.1, $\eta(H) \geq \tau(H) \geq k$. We argue by contradiction to prove the necessity. Suppose that $\eta(H)=k$. Let $\left(l_{1}, l_{2}, \cdots, l_{m}\right)$ and the sequence $J_{1}, J_{2}, \cdots, J_{m}$ be the $\eta$ spectrum and the $\eta$-decomposition of $H$. By Corollary 6.4.10, $d\left(H / J_{2}\right)=\eta\left(H / J_{2}\right)=\gamma\left(H / J_{2}\right)=$ $\eta(H)=k$. By (i), for any $E^{\prime} \in \mathcal{E}\left(H / J_{2}\right)$ and its preimage $E \in \mathcal{E}(H), \tau\left((H-E) / J_{2}\right)=$ $\tau\left(H / J_{2}-E^{\prime}\right)<k$. By Proposition 6.4.1(C2), $\tau(H-E)<k$, contrary to $\mathcal{E}_{k}(H)=\mathcal{E}(H)$. This proves the necessity.

We argue by contradiction to prove the sufficiency. Let $H$ be a hypergraph with

$$
\eta(H)>k \text { and } \mathcal{E}_{k}(H) \neq \mathcal{E}(H) \text { such that } V(H) \text { is minimized. }
$$

Since $\mathcal{E}_{k}(H) \neq \mathcal{E}(H)$, there exists $E_{0} \in \mathcal{E}(H)$ such that

$$
\tau\left(H-E_{0}\right) \leq k-1 .
$$

Claim 3. For any nontrivial sub-hypergraph $S$ of $H$ with $|V(S)|<|V(H)|, \eta(S) \leq k$.

Proof of Claim 3. Suppose not and we have $\eta(S)>k$. By $(6.11), \mathcal{E}_{k}(S)=\mathcal{E}(S)$. By Lemma 6.3.4(i), $\eta(H / S) \geq \eta(S)>k$, and so by $(6.11), \mathcal{E}_{k}(H / S)=\mathcal{E}(H / S)$. It follows from Lemma 6.6.1 that $\mathcal{E}_{k}(H)=\mathcal{E}(H)$, contrary to (6.11). This proves Claim 3.

By Claim 3, for any $S \subseteq \mathcal{E}(H), \eta(S) \leq k<\eta(H)$. By Lemma 6.3.5(ii), $H$ is uniformly dense. Then $d(H)=\eta(H)>k$, and so $|\mathcal{E}(H)| \geq k(|V(H)|-1)+1$. We have $d\left(H-E_{0}\right)=$ $\frac{\left|\mathcal{E}\left(H-E_{0}\right)\right|}{\left|V\left(H-E_{0}\right)\right|-\omega\left(H-E_{0}\right)} \geq \frac{|\mathcal{E}(H)|-1}{|V(H)|-1} \geq k$. By Lemma 6.3.4(iii), there exists a nonempty subset $X \subseteq$ $\mathcal{E}\left(H-E_{0}\right)$ such that $\eta\left(\left(H-E_{0}\right)[X]\right) \geq k$. Thus $\tau\left(\left(H-E_{0}\right)[X]\right)=\left\lfloor\eta\left(\left(H-E_{0}\right)[X]\right)\right\rfloor \geq k$.

By Lemma 6.3.4(i), $\eta(H / X) \geq \eta(H)>k$. Let $E_{0}^{\prime} \in \mathcal{E}(H / X)$. By (6.11), $H$ is a minimal counterexample, and so $\tau\left(H / X-E_{0}^{\prime}\right) \geq k$. Thus $\tau\left(\left(H-E_{0}\right) / X\right)=\tau\left(H / X-E_{0}^{\prime}\right) \geq k$. As $\tau\left(\left(H-E_{0}\right)[X]\right) \geq k$, by Proposition 6.4.1(C3), $\tau\left(H-E_{0}\right) \geq k$. contrary to (6.12). This completes the proof of the sufficiency.

(iii) Suppose that $\eta(H)=k$. If $d(H)=k$, then by (i), $\mathcal{E}_{k}(H)=\emptyset$. On the other hand, by Theorem 6.3.3, $H$ is uniformly dense. By Corollary 6.4.11, $m=1$ and so $J_{2}=\emptyset$. Thus $\mathcal{E}_{k}(H)=$ $J_{2}$. If $d(H)>k$, then $H$ is not uniformly dense, and by Corollary 6.4.11, $m>1$. Suppose that $H$ has $\eta$-spectrum (6.9) and $\eta$-decomposition (6.10). By Theorem 6.4.9, $\eta\left(H\left[J_{2}\right]\right)=l_{2}>l_{1}=$ $\eta(H)=k$. It follows from (ii) that $\mathcal{E}_{k}\left(H\left[J_{2}\right]\right)=J_{2}$. By Corollary 6.4.10, $H / J_{2}$ is uniformly dense with $\eta\left(H / J_{2}\right)=d\left(H / J_{2}\right)=k$, and so by (i), $\mathcal{E}_{k}\left(H / J_{2}\right)=\emptyset$. Then for any hyperedge $E \in J_{2}, \tau\left((H-E)\left[J_{2}-E\right]\right)=\tau\left(H\left[J_{2}\right]-E\right)=k$ and $\tau\left((H-E) /\left(J_{2}-E\right)\right)=\tau\left(H / J_{2}\right)=k$. By Proposition 6.4.1(C3), $\tau(H-E)=k$. Thus $J_{2} \subseteq \mathcal{E}_{k}(H)$. To complete the proof, we still need to show that $\mathcal{E}_{k}(H) \subseteq J_{2}$. It suffices to prove that for any $E \in \mathcal{E}(H) \backslash J_{2}, \tau(H-E)<k$. If not, we have $\tau(H-E)=k$ and let $E^{\prime} \in \mathcal{E}\left(H / J_{2}\right)$ be the image of $E$, and by Proposition 6.4.1(C2), $\tau\left(H / J_{2}-E^{\prime}\right)=\tau\left((H-E) / J_{2}\right)=k$, contrary to $\mathcal{E}_{k}\left(H / J_{2}\right)=\emptyset$. Hence $\mathcal{E}_{k}(H)=J_{2}$. 


\section{Bibliography}

[1] C. Berge, Hypergraphs: combinatorics of finite sets, North-Holland, 1989.

[2] D. Billington, Lattices and degree sequences of uniform hypergraphs, Ars Combin., 21-A (1986), 9-19.

[3] F. T. Boesch and S. Chen, A generalization of line connectivity and optimally invulnerable graphs, SIAM J. Appl. Math, 34 (1978), 657-665.

[4] E. T. Boesch and J. A. M. McHugh, An edge extremal result for subcohesion, J. Combinat. Theory Ser. B, 38 (1985), 1-7.

[5] B. Bollobás, Extremal Graph Theory, Dover Publications, New York, 2004.

[6] J. A. Bondy and U. S. R. Murty, Graph Theory, Springer, New York, 2008.

[7] V. Boonyasombat, Degree sequences of connected hypergraphs and hypertrees, Lecture Notes in Mathematics, 1073 (1984), 236-247.

[8] A. E. Brouwer and W. H. Haemers, Spectra of Graphs, Springer Universitext, 2012. Available from: http://homepages.cwi.nl/ aeb/math/ipm/ipm.pdf

[9] P. A. Catlin, The reduction of graph families under contraction, Discrete Mathematics, 160 (1996), 67-80.

[10] P. A. Catlin, J. W. Grossman, A. M. Hobbs, Graphs with uniform density, Congr. Numer., 65 (1988), 281-286.

[11] P. A. Catlin, J. W. Grossman, A. M. Hobbs and H.-J. Lai, Fractional arboricity, strength and principal partitions in graphs and matroids, Discrete Appl. Math. 40 (1992) 285-302.

[12] P. A. Catlin, A. M. Hobbs and H.-J. Lai, Graph families operations, Discrete Math, 230 (2001), 71-97.

[13] G. Chartrand, S. F. Kapoor, L. Lesniak and D. R. Lick, Generalized connectivity in graphs, Bull. Bombay Math. Colloq. 2 (1984), 1-6. 
[14] G. Chaty and M. Chein, Minimally 2-edge-connected graphs. J. Graph Theory, 3 (1979), $15-22$.

[15] S. A. Choudum, On graphic and 3-hypergraphic sequences, Discrete Mathematics, 87 (1991), 91-95.

[16] S. M. Cioabă, The spectral radius and the maximum degree of irregular graphs, Electron. J. Combin., 14 (2007), R38.

[17] S. M. Cioabă, Eigenvalues and edge-connectivity of regular graphs, Linear algebra Appl., 432 (2010), 458-470.

[18] S. M. Cioabă, D. A. Gregory, and V. Nikiforov, Note: extreme eigenvalues of nonregular graphs, J. Combin. Theory Ser. B, 97 (2007), 483-486.

[19] S. M. Cioabă and W. Wong, Edge-disjoint spanning trees and eigenvalues of regular graphs, Linear Algebra Appl., 437 (2012), 630-647.

[20] C. J. Colbourn, W. L. Kocay and D. R. Stinson, Some NP-complete problems for hypergraph degree sequences, Discrete Appl. Math., 14 (1986), 239-254.

[21] W. H. Cunningham, Optimal attack and reinforcement of a network, Journal of the ACM, 32 (1985), 549-561.

[22] A. Dewdney, Degree sequences in complexes and hypergraphs, Proc. Amer. Math. Soc, 53 (1975), 535-540.

[23] G. A. Dirac, Minimally 2-connected graphs, J. Reine Angew. Math., 228 (1967), 204-216.

[24] J. Edmonds, Existence of $k$-edge connected ordinary graphs with prescribed degree, J. Res. Nat. Bur. Stand. Ser. B, 68 (1964), 73-74.

[25] J. Edmonds, Lehman's switching game and a theorem of Tutte and Nash-Williams, J. Res. Nat. Bur. Standards Sect. B, 69 (1965), 73-77.

[26] P. Erdős, Personal reminiscence and remarks on the mathematical work of Tibor Gallai, Combinatorica, 2 (1982), 207-212.

[27] A. Frank, Augmenting graphs to meet edge-connectivity requirements, SIAM J. Discrete Math., 5 (1992), 22-53.

[28] A. Frank and T. Király, Combined connectivity augmentation and orientation problems, Discrete Applied Math., 131 (2003), 401-419. 
[29] A. Frank, T. Király and Matthias Kriesell, On decomposing a hypergraph into $k$ connected sub-hypergraphs, Discrete Applied Mathematics, 131 (2003), 373-383.

[30] D. Gale, A theorem on flows in networks, Pacific Journal of Mathematics, 7 (1957), 10731082.

[31] C. Godsil and G. Royle, Algebraic Graph Theory, Springer-Verlag, New York, 2001.

[32] D. L. Goldsmith, On the second order edge-connectivity of a graph, Congressus Numerantium, 29 (1980), 479-484.

[33] D. L. Goldsmith, On the $n$th order edge-connectivity of a graph, Congressus Numerantium, 32 (1981), 375-382.

[34] X. Gu and H. -J. Lai, Realizing degree sequences with $k$-edge-connected uniform hypergraphs, Discrete Mathematics, 313 (2013), 1394-1400.

[35] X. Gu, H. -J. Lai and S. Yao, Characterization of minimally $(2, l)$-connected graphs, Information Processing Letters, 111 (2011), 1124-1129.

[36] D. Gusfield, Connectivity and edge-disjoint spanning trees, Information Processing Letters, 16 (1983), 87-89.

[37] R. Haas, Characterizations of arboricity of graphs, Ars Combinatoria, 63 (2002), 129-137.

[38] W. H. Haemers, Interlacing eigenvalues and graphs, Linear Algebra Appl., 226-228 (1995), 593-616.

[39] K. Hennayake, H.-J. Lai, D. Li and J. Mao, Minimally $(k, k)$-edge-connected graphs, J. Graph Theory, 44 (2003), 116-131.

[40] K. Hennayake, H.-J. Lai and L. Xu, The strength and the l-edge-connectivity of a graph, Bull. Inst. Comb. Appl., 26 (1999), 55-70.

[41] T. Jordán, Minimax theorems in graph connectivity augmentation, EGRES Tech. Rep. 2012-14.

[42] T. Király and M. Makai, A note on hypergraph connectivity augmentation, EGRES Tech. Rep. 2002-11.

[43] H. -J. Lai, The size of strength-maximal graphs, Journal of Graph Theory, 14 (1990), 187197.

[44] H.-J. Lai and H. Y. Lai, Duality of graph families, Discrete Mathematics, 110 (1992), 165-177. 
[45] H. -J. Lai, P. Li, Y. Liang and J. Xu, Reinforcing a matroid to have $k$ disjoint bases, Applied Mathematics, 1 (2010), 244-249.

[46] P. Li, Cycles and bases of graphs and matroids, Ph.D. dissertation, West Virginia University, 2012 .

[47] P. Li, H. -J. Lai and Y. Liang, Characterization of removable elements with respect to having $k$ disjoint bases in a matroid, Discrete Applied Mathematics, 160 (2012), 2445-2451.

[48] D. Liu, H. -J. Lai and Z.-H. Chen, Reinforcing the number of disjoint spanning trees, Ars Combinatoria, 93 (2009), 113-127.

[49] B. L. Liu and G. Li, A note on the largest eigenvalue of non-regular graphs, Electronic Journal of Linear Algebra, 17 (2008), 54-61.

[50] B. L. Liu, J. Shen, and X. M. Wang, On the largest eigenvalue of non-regular graphs, J. Combin. Theory Ser. B, 97 (2007), 1010-1018.

[51] M. Lorea, Hypergraphes et matroides, Cahiers Centre Etudes Rech. Oper., 17 (1975), 289291.

[52] W. Mader, Minimale $n$-fach kantenzusammenhängende graphen, Math. Ann., 191 (1971), 21-28.

[53] D. Matula, K-components, clusters, and slicings in graphs, SIAM J. Appl. Math., 22 (1972), 459-480.

[54] D. Matula, Determining edge connectivity in $O(m n)$, Proceedings of 28th Symp. on Foundations of Computer Science, (1987), 249-251.

[55] H. Minc, Nonnegative Matrices, John Wiley and Sons, New York, 1988.

[56] J. Mitchem, An extension of Brooks' theorem to $n$-degenerate graphs, Discrete Math., 17 (1977), 291-298

[57] C. St. J. A. Nash-williams, Edge-disjoint spanning trees of finite graphs, J. London Math. Soc., 36 (1961), 445-450.

[58] C. St. J. A. Nash-williams, Decompositions of finite graphs into forests, J. London Math. Soc., 39 (1964), 12.

[59] O. R. Oellermann, Explorations into graph connectivity, The Notices of the South African Mathematical Society, 20 (1988), 117-151. 
[60] O. R. Oellermann, Generalized Connectivity in Graphs, Ph.D. Dissertation, Western Michigan University, 1986.

[61] O. R. Oellermann, On the l-connectivity of a graph, Graphs and Combinatorics, 3 (1987), 285-291.

[62] J. G. Oxley, Matroid Theory, 2 edition, Oxford University Press, New York, 2011.

[63] E. M. Palmer, On the spanning tree packing number of a graph: a survey, Discrete Math., 230 (2001), 13-21.

[64] M. D. Plummer, On minimal blocks, Transactions of the American Mathematical Society, 134 (1968), 85-94.

[65] H. J. Ryser, Combinatorial properties of matrices of zeros and ones, Canad. J. Math., 9 (1957), 371-377.

[66] W. So, Commutativity and spectra of Hermitian matrices, 212-213 (1994), 121-129.

[67] D. Stevanović. The largest eigenvalue of nonregular graphs, J. Combin. Theory Ser. B, 91 (2004), 143-146.

[68] G. Strang, Introduction to Linear Algebra, 4th Edition, Wellesley-Cambridge Press, 2009.

[69] Z. Szigeti, On edge-connectivity augmentations of graphs and hypergraphs, In: W. Cook, L. Lovász and J. Vygen (eds.), Research trends in combinatorial optimization. Springer, Berlin, 2009, 483-521.

[70] W. T. Tutte, On the problem of decomposing a graph into $n$ factors, J. London Math. Soc., 36 (1961), 221-230.

[71] T. Watanabe and A. Nakamura, Edge-connectivity augmentation problems, J. Comput. Syst. Sci., 35 (1987), 96-144.

[72] X.D. Zhang, Eigenvectors and eigenvalues of non-regular graphs, Linear Algebra Appl., 409 (2005), 79-86.

[73] L. Zhang, K. Hennayake, H.-J. Lai and Y. Shao, A lower bound of the l-edge-connectivity and optimal graphs, J. Combin. Math. Combin. Comput., 66 (2008), 79-95. 